\title{
Research on intuitionistic fuzzy implications
}

\author{
Nora Angelova $^{1}$ and Krassimir T. Atanassov ${ }^{2,3}$ \\ ${ }^{1}$ Faculty of Mathematics and Informatics, Sofia University \\ 5 James Bourchier Blvd., 1164 Sofia, Bulgaria \\ e-mail: noraa@fmi.uni-sofia.bg \\ 2 Dept. of Bioinformatics and Mathematical Modelling \\ Institute of Biophysics and Biomedical Engineering \\ Bulgarian Academy of Sciences \\ 105 Acad. G. Bonchev Str., 1113 Sofia, Bulgaria \\ ${ }^{3}$ Intelligent Systems Laboratory \\ Prof. Dr. Asen Zlatarov University, 8010 Burgas, Bulgaria \\ e-mail: kratebas.bg
}

Received: 21 January 2021

Accepted: 8 June 2021

\begin{abstract}
Currently in the theories of intuitionistic fuzzy sets, logics and pairs, there are 198 different implications. Here, we check the relationships between every two of them.
\end{abstract}

Keywords: Intuitionistic fuzzy implication, Intuitionistic fuzzy pair, Intuitionistic fuzzy set.

2020 Mathematics Subject Classification: 03 E72.

\section{Introduction}

Following and extending [16], here we give short remarks on the results related to the intuitionistic fuzzy implications. In Section 2, we give the complete Table of the relations between the separate implications. Such research is done for the first time in fuzzy sets theory.

In the series of papers [2-4,7-11,14,15,21,22,24,32-48,50-52,55-57], different implications were defined and some of their basic properties were studied. In some of these publications, some misprints in the formulas were found during the last years. Here, we give the full list of the corrected intuitionistic fuzzy implications.

The first two implications were introduced in [5] by K. Atanassov. Fifteen years ago, the first 10 implications, described in [7] were constructed as intuitionistic fuzzy analogues of existing 
fuzzy implications in literature (see, e.g., [54]). At that moment, K.A. understood that his first implications coincide with the intuitionistic fuzzy analogue of Kleene-Dienes's implication, while the second one is an extension of the intuitionistic fuzzy analogue of Gödel's implication.

The next five implications were introduced in K.Atanassov's publications [8, 22, 32, 33]. Two of them are given in papers, written together with B. Kolev [22] $\left(\rightarrow_{13}\right)$ and T. Trifonov $\left(\rightarrow_{14}\right)$ $[32,33]$. From the so constructed 15 implications, using the formula

$$
\neg A=A \rightarrow F
$$

( $F$ is the logical false), five negations are formulated - the standard (classical) negation $\neg_{1}$ and four others $\neg_{2}, \ldots, \neg_{5}$, where $\rightarrow$ is each one of these 15 implications [6]. By formulas

$$
A \rightarrow B=\neg A \vee B
$$

and

$$
A \rightarrow B=\neg A \vee \neg \neg B,
$$

these 5 negations generate eight new implications [9]. It is important to note that negations $\neg_{2}, \ldots, \neg_{5}$ do not satisfy equalities

$$
A=\neg \neg A
$$

and

$$
\begin{aligned}
& A \wedge B=\neg(\neg A \vee \neg B), \\
& A \vee B=\neg(\neg A \wedge \neg B),
\end{aligned}
$$

but they satisfy equalities

$$
\begin{aligned}
& \neg \neg A \wedge \neg \neg B=\neg(\neg A \vee \neg B), \\
& \neg \neg A \vee \neg \neg B=\neg(\neg A \wedge \neg B) .
\end{aligned}
$$

Each of these 23 implications generates four new implications, by formulas using intuitionistic fuzzy (standard, classical) modal operators (see, e.g. [53]). The analogues of these implications are given in [13].

In [38-41], L. Atanassova introduced 11 new implications $\left(\rightarrow_{139}, \ldots, \rightarrow_{149}\right)$. P. Dworniczak generalized them in [50-52] (implications $\rightarrow_{150}, \ldots, \rightarrow_{152}$ ) and then L. Atanassova modified Dworniczak's implications in [45-47] (implications $\rightarrow_{154}, \ldots, \rightarrow_{165}$ ).

The next five implications were introduced by the author in [12] as modifications of the first L. Zadeh's type intuitionistic fuzzy implication $\rightarrow_{1}$, implications $\rightarrow_{171}, \rightarrow_{175}$ were introduced by the author in [15].

Implications $\rightarrow_{176}, \ldots, \rightarrow_{180}$ were proposed in $[56,57]$ by E. Szmidt, J. Kacprzyk and K. Atanassov, while the last five implications $\rightarrow_{181}, \ldots, \rightarrow_{185}$ were introduced by K. Atanassov in [15]. Again E. Szmidt, J. Kacprzyk and K. Atanassov introduced implications $\rightarrow_{186}, \rightarrow_{187}$, and $\rightarrow_{188}$ in [25, 27-30]. L. Atanassova defined implication $\rightarrow_{189}$ in $[48,49]$ and S. Ribagin, L. Dukovska, V. Atanassova and K. Atanassov - implication $\rightarrow_{190}$ in [23].

In [16], it is shown which implications between $\rightarrow_{1}$ and $\rightarrow_{195}$ satisfy the axioms of Klir and Yuan, of Kolmogorov's and of Łukasiewisz-Tarski's axioms of classical logic and of intuitionistic logic. 
Implication $\rightarrow_{191}$ called Goguen intuitionistic logic implication was introduced by the authors together with V. Atanassova in [20]. Implication $\rightarrow_{192}$ called Third Zadeh's intuitionistic logic implication was introduced by K. A. in [17] and it was modified to 6 new implications $\rightarrow_{193}$ $, \ldots, \rightarrow_{198}$ in [18] by the two authors.

All currently existing implications are given in Table 1 . In it, we keep the numeration from $[13,16,58]$.

We must mention that in some formulas we use the functions

$$
\operatorname{sg}(x)=\left\{\begin{array}{cc}
1 & \text { if } x>0 \\
0 & \text { if } x \leq 0
\end{array}, \quad \overline{s g}(x)=\left\{\begin{array}{cc}
0 & \text { if } x>0 \\
1 & \text { if } x \leq 0
\end{array}\right.\right.
$$

Table 1. List of the intuitionistic fuzzy implications

\begin{tabular}{|c|c|}
\hline$\rightarrow_{1}$ & $\langle\max (b, \min (a, c)), \min (a, d)\rangle$ \\
\hline$\rightarrow_{2}$ & $\langle\overline{s g}(a-c), d \operatorname{sg}(a-c)\rangle$ \\
\hline$\rightarrow_{3}$ & $\langle 1-(1-c) \operatorname{sg}(a-c)), d \operatorname{sg}(a-c)\rangle$ \\
\hline$\rightarrow_{4}$ & $\langle\max (b, c), \min (a, d)\rangle$ \\
\hline$\rightarrow_{5}$ & $\langle\min (1, b+c), \max (0, a+d-1)\rangle$ \\
\hline$\rightarrow_{6}$ & $\langle b+a c, a d\rangle$ \\
\hline$\rightarrow_{7}$ & $\begin{array}{l}\langle\min (\max (b, c), \max (a, b), \max (c, d)), \\
\max (\min (a, d), \min (a, b), \min (c, d))\rangle\end{array}$ \\
\hline$\rightarrow_{8}$ & $\langle 1-(1-\min (b, c)) \operatorname{sg}(a-c), \max (a, d) \operatorname{sg}(a-c), \operatorname{sg}(d-b)\rangle$ \\
\hline$\rightarrow_{9}$ & $\left\langle b+a^{2} c, a b+a^{2} d\right\rangle$ \\
\hline$\rightarrow 10$ & $\begin{array}{c}\langle c \overline{s g}(1-a)+\operatorname{sg}(1-a)(\overline{s g}(1-c)+b \operatorname{sg}(1-c)) \\
d \overline{s g}(1-a)+a \operatorname{sg}(1-a) \operatorname{sg}(1-c)\rangle\end{array}$ \\
\hline$\rightarrow_{11}$ & $\langle 1-(1-c) \operatorname{sg}(a-c), d \operatorname{sg}(a-c) \operatorname{sg}(d-b)\rangle$ \\
\hline$\rightarrow_{12}$ & $\langle\max (b, c), 1-\max (b, c)\rangle$ \\
\hline$\rightarrow_{13}$ & $\langle b+c-b c, a d\rangle$ \\
\hline$\rightarrow_{14}$ & $\langle 1-(1-c) \operatorname{sg}(a-c)-d \overline{s g}(a-c) \operatorname{sg}(d-b), d \operatorname{sg}(d-b)\rangle$ \\
\hline$\rightarrow_{15}$ & $\begin{array}{c}\langle 1-(1-\min (b, c)) \operatorname{sg}(\operatorname{sg}(a-c)+\operatorname{sg}(d-b)) \\
-\min (b, c) \operatorname{sg}(a-c) \operatorname{sg}(d-b), 1-(1-\max (a, d)) \operatorname{sg}(\overline{s g}(a-c) \\
+\overline{s g}(d-b))-\max (a, d) \overline{s g}(a-c) \overline{s g}(d-b)\rangle\end{array}$ \\
\hline$\rightarrow_{16}$ & $\langle\max (\overline{s g}(a), c), \min (\operatorname{sg}(a), d)\rangle$ \\
\hline$\rightarrow_{17}$ & $\left\langle\max (b, c), \min \left(a b+a^{2}, d\right)\right\rangle$ \\
\hline$\rightarrow_{18}$ & $\langle\max (b, c), \min (1-b, d)\rangle$ \\
\hline$\rightarrow_{19}$ & $\langle\max (1-\operatorname{sg}(\operatorname{sg}(a)+\operatorname{sg}(1-b)), c), \min (\operatorname{sg}(1-b), d)\rangle$ \\
\hline$\rightarrow_{20}$ & $\langle\max (\overline{s g}(a), \operatorname{sg}(c)), \min (\operatorname{sg}(a), \overline{s g}(c))\rangle$ \\
\hline$\rightarrow_{21}$ & $\left\langle\max (b, c(c+d)), \min \left(a(a+b), d\left(c^{2}+d+c d\right)\right)\right\rangle$ \\
\hline$\rightarrow_{22}$ & $\langle\max (b, 1-d), 1-\max (b, 1-d)\rangle$ \\
\hline$\rightarrow_{23}$ & $\langle 1-\min (\operatorname{sg}(1-b), \overline{s g}(1-d)), \min (\operatorname{sg}(1-b), \overline{s g}(1-d))\rangle$ \\
\hline
\end{tabular}

(Continued on next page) 
Table 1 (Continued from previous page)

\begin{tabular}{|c|c|}
\hline$\rightarrow_{24}$ & $\langle\overline{s g}(a-c) \overline{s g}(d-b), \operatorname{sg}(a-c) \operatorname{sg}(d-b)\rangle$ \\
\hline$\rightarrow_{25}$ & $\langle\max (b, \overline{s g}(a) \overline{s g}(1-b)), c \overline{s g}(d) \overline{s g}(1-c)), \min (a, d)\rangle$ \\
\hline$\rightarrow_{26}$ & $\langle\max (\overline{s g}(1-b), c), \min (\operatorname{sg}(a), d)\rangle$ \\
\hline$\rightarrow_{27}$ & $\langle\max (\overline{s g}(1-b), \operatorname{sg}(c)), \min (\operatorname{sg}(a), \overline{s g}(1-d))\rangle$ \\
\hline$\rightarrow_{28}$ & $\langle\max (\overline{s g}(1-b), c), \min (a, d)\rangle$ \\
\hline$\rightarrow_{29}$ & $\langle\max (\overline{s g}(1-b), \overline{s g}(1-c)), \min (a, \overline{s g}(1-d))\rangle$ \\
\hline$\rightarrow_{30}$ & $\langle\max (1-a, \min (a, 1-d)), \min (a, d)\rangle$ \\
\hline$\rightarrow_{31}$ & $\langle\overline{s g}(a+d-1), d \operatorname{sg}(a+d-1)\rangle$ \\
\hline$\rightarrow_{32}$ & $\langle 1-d \operatorname{sg}(a+d-1), d \operatorname{sg}(a+d-1)\rangle$ \\
\hline$\rightarrow_{33}$ & $\langle 1-\min (a, d), \min (a, d)\rangle$ \\
\hline$\rightarrow_{34}$ & $\langle\min (1,2-a-d), \max (0, a+d-1)\rangle$ \\
\hline$\rightarrow_{35}$ & $\langle 1-a d, a d\rangle$ \\
\hline$\rightarrow_{36}$ & $\begin{array}{c}\langle\min (1-\min (a, d), \max (a, 1-a), \max (1-d, d)), \max (\min (a, d) \\
\min (a, 1-a), \min (1-d, d))\rangle\end{array}$ \\
\hline$\rightarrow_{37}$ & $\langle 1-\max (a, d) \operatorname{sg}(a+d-1), \max (a, d) \operatorname{sg}(a+d-1)\rangle$ \\
\hline$\rightarrow_{38}$ & $\left\langle 1-a+a^{2}(1-d), a(1-a)+a^{2} d\right\rangle$ \\
\hline$\rightarrow_{39}$ & $\begin{array}{c}\langle(1-d) \overline{s g}(1-a)+\operatorname{sg}(1-a)(\overline{s g}(d)+(1-a) \operatorname{sg}(d)) \\
d \overline{s g}(1-a)+a \operatorname{sg}(1-a) \operatorname{sg}(d)\rangle\end{array}$ \\
\hline$\rightarrow_{40}$ & $\langle 1-\operatorname{sg}(a+d-1), 1-\overline{s g}(a+d-1)\rangle$ \\
\hline$\rightarrow_{41}$ & $\langle\max (\overline{s g}(a), 1-d), \min (\operatorname{sg}(a), d)\rangle$ \\
\hline$\rightarrow_{42}$ & $\langle\max (\overline{s g}(a), \operatorname{sg}(1-d)), \min (\operatorname{sg}(a), \overline{s g}(1-d))\rangle$ \\
\hline$\rightarrow_{43}$ & $\langle\max (\overline{s g}(a), 1-d), \min (\operatorname{sg}(a), d)\rangle$ \\
\hline$\rightarrow_{44}$ & $\langle\max (\overline{s g}(a), 1-d), \min (a, d)\rangle$ \\
\hline$\rightarrow_{45}$ & $\langle\max (\overline{s g}(a), \overline{s g}(d)), \min (a, \overline{s g}(1-d))\rangle$ \\
\hline$\rightarrow_{46}$ & $\langle\max (b, \min (1-b, c)), 1-\max (b, c)\rangle$ \\
\hline$\rightarrow_{47}$ & $\langle\overline{s g}(1-b-c),(1-c) \operatorname{sg}(1-b-c)\rangle$ \\
\hline$\rightarrow_{48}$ & $\langle 1-(1-c) \operatorname{sg}(1-b-c),(1-c) \operatorname{sg}(1-b-c)\rangle$ \\
\hline$\rightarrow_{49}$ & $\langle\min (1, b+c), \max (0,1-b-c)\rangle$ \\
\hline$\rightarrow_{50}$ & $\langle b+c-b c, 1-b-c+b c\rangle$ \\
\hline$\rightarrow_{51}$ & $\begin{array}{c}\langle\min (\max (b, c), \max (1-b, b), \max (c, 1-c)) \\
\max (1-\max (b, c), \min (1-b, b), \min (c, 1-c))\rangle\end{array}$ \\
\hline$\rightarrow_{52}$ & $\langle 1-(1-\min (b, c)) \operatorname{sg}(1-b-c), 1-\min (b, c) \operatorname{sg}(1-b-c)\rangle$ \\
\hline$\rightarrow_{53}$ & $\left\langle b+(1-b)^{2} c,(1-b) b+(1-b)^{2}(1-c)\right\rangle$ \\
\hline$\rightarrow_{54}$ & $\begin{array}{l}\langle c \overline{s g}(b)+\operatorname{sg}(b)(\overline{s g}(1-c)+b \operatorname{sg}(1-c)) \\
\quad(1-c) \overline{s g}(b)+(1-b) \operatorname{sg}(b) \operatorname{sg}(1-c)\rangle\end{array}$ \\
\hline$\rightarrow_{55}$ & $\langle 1-\operatorname{sg}(1-b-c), 1-\overline{s g}(1-b-c)\rangle$ \\
\hline$\rightarrow_{56}$ & $\langle\max (\overline{s g}(1-b), c), \min (\operatorname{sg}(1-b), 1-c)\rangle$ \\
\hline$\rightarrow_{57}$ & $\langle\max (\overline{s g}(1-b), \operatorname{sg}(c)), \min (\operatorname{sg}(1-b), \overline{s g}(c))\rangle$ \\
\hline
\end{tabular}


Table 1 (Continued from previous page)

\begin{tabular}{|c|c|}
\hline$\rightarrow_{58}$ & $\langle\max (\overline{s g}(1-b), \overline{s g}(1-c)), 1-\max (b, c)\rangle$ \\
\hline$\rightarrow_{59}$ & $\langle\max (\overline{s g}(1-b), c), 1-\max (b, c)\rangle$ \\
\hline$\rightarrow_{60}$ & $\langle\max (\overline{s g}(1-b), \overline{s g}(1-c)), \min (1-b, \overline{s g}(c))\rangle$ \\
\hline$\rightarrow_{61}$ & $\langle\max (c, \min (b, d)), \min (a, d)\rangle$ \\
\hline$\rightarrow_{62}$ & $\langle\overline{s g}(d-b), a \operatorname{sg}(d-b)\rangle$ \\
\hline$\rightarrow_{63}$ & $\langle 1-(1-b) \operatorname{sg}(d-b), a \operatorname{sg}(d-b)\rangle$ \\
\hline$\rightarrow_{64}$ & $\langle c+b d, a d\rangle$ \\
\hline$\rightarrow_{65}$ & $\langle 1-(1-\min (b, c)) \operatorname{sg}(d-b), \max (a, d) \operatorname{sg}(d-b) \operatorname{sg}(a-c)\rangle$ \\
\hline$\rightarrow_{66}$ & $\left\langle c+d^{2} b, b d+d^{2} a\right\rangle$ \\
\hline$\rightarrow_{67}$ & $\begin{array}{c}\langle b \overline{s g}(1-d)+\operatorname{sg}(1-d)(\overline{s g}(1-b)+c \operatorname{sg}(1-b)) \\
a \overline{s g}(1-d)+d \operatorname{sg}(1-d) \operatorname{sg}(1-b)\rangle\end{array}$ \\
\hline$\rightarrow_{68}$ & $\langle 1-(1-b) \operatorname{sg}(d-b), a \operatorname{sg}(d-b) \operatorname{sg}(a-c)\rangle$ \\
\hline$\rightarrow_{69}$ & $\langle 1-(1-b) \operatorname{sg}(d-b)-a \overline{s g}(d-b) \operatorname{sg}(a-c), a \operatorname{sg}(a-c)\rangle$ \\
\hline$\rightarrow_{70}$ & $\langle\max (\overline{s g}(d), b), \min (\operatorname{sg}(d), a)\rangle$ \\
\hline$\rightarrow_{71}$ & $\left\langle\max (b, c), \min \left(c d+d^{2}, a\right)\right\rangle$ \\
\hline$\rightarrow_{72}$ & $\langle\max (b, c), \min (1-c, a)\rangle$ \\
\hline$\rightarrow_{73}$ & $\langle\max (1-\max (\operatorname{sg}(d), \operatorname{sg}(1-c)), b), \min (\operatorname{sg}(1-c), a)\rangle$ \\
\hline$\rightarrow_{74}$ & $\langle\max (\operatorname{sg}(b), \overline{s g}(d)), \min (\overline{s g}(b), \operatorname{sg}(d))\rangle$ \\
\hline$\rightarrow_{75}$ & $\left\langle\max (c, b(a+b)), \min \left(d(c+d), a\left(b^{2}+a\right)+a b\right)\right\rangle$ \\
\hline$\rightarrow_{76}$ & $\langle\max (c, 1-a), \min (1-c, a)\rangle$ \\
\hline$\rightarrow 77$ & $\langle(1-\min (\overline{s g}(1-a), \operatorname{sg}(1-c))), \min (\overline{s g}(1-a), \operatorname{sg}(1-c))\rangle$ \\
\hline$\rightarrow 78$ & $\langle\max (\overline{s g}(1-c), b), \min (\operatorname{sg}(d), a)\rangle$ \\
\hline$\rightarrow 79$ & $\langle\max (\overline{s g}(1-c), \operatorname{sg}(b)), \min (\operatorname{sg}(d), \overline{s g}(1-a))\rangle$ \\
\hline$\rightarrow_{80}$ & $\langle\max (\overline{s g}(1-c), b), \min (d, a)\rangle$ \\
\hline$\rightarrow_{81}$ & $\langle\max (\overline{s g}(1-b), \overline{s g}(1-c)), \min (d, \overline{s g}(1-a))\rangle$ \\
\hline$\rightarrow_{82}$ & $\langle\max (1-d, \min (d, 1-a)), \min (d, a)\rangle$ \\
\hline$\rightarrow_{83}$ & $\langle\overline{s g}(a+d-1), a \operatorname{sg}(a+d-1)\rangle$ \\
\hline$\rightarrow_{84}$ & $\langle 1-\operatorname{asg}(a+d-1), a \operatorname{sgg}(a+d-1)\rangle$ \\
\hline$\rightarrow_{85}$ & $\left\langle 1-d+d^{2}(1-a), d(1-d)+d^{2} a\right\rangle$ \\
\hline$\rightarrow_{86}$ & $\begin{array}{c}\langle(1-a) \overline{s g}(1-d)+\operatorname{sg}(1-d)(\overline{s g}(a)+(1-d) \operatorname{sg}(d)) \\
a \overline{s g}(1-d)+d \operatorname{sg}(1-d) \operatorname{sg}(a)\rangle\end{array}$ \\
\hline$\rightarrow_{87}$ & $\langle\max (\overline{s g}(d), 1-a), \min (\operatorname{sg}(d), a)\rangle$ \\
\hline$\rightarrow_{88}$ & $\langle\max (\overline{s g}(d), \operatorname{sg}(1-a)), \min (\operatorname{sg}(d), \overline{s g}(1-a))\rangle$ \\
\hline$\rightarrow_{89}$ & $\langle\max (\overline{s g}(d), 1-a), \min (d, a)\rangle$ \\
\hline$\rightarrow_{90}$ & $\langle\max (\overline{s g}(a), \overline{s g}(d)), \min (d, \overline{s g}(1-a))\rangle$ \\
\hline$\rightarrow_{91}$ & $\langle\max (c, \min (1-c, b)), 1-\max (b, c)\rangle$ \\
\hline$\rightarrow_{92}$ & $\langle\overline{s g}(1-b-c), \min (1-b, \operatorname{sg}(1-b-c))\rangle$ \\
\hline$\rightarrow_{93}$ & $\langle(1-\min (1-b, \operatorname{sg}(1-b-c)), \min (1-b, \operatorname{sg}(1-b-c))\rangle$ \\
\hline
\end{tabular}

(Continued on next page) 
Table 1 (Continued from previous page)

\begin{tabular}{|c|c|}
\hline$\rightarrow_{94}$ & $\left\langle c+(1-c)^{2} b,(1-c) c+(1-c)^{2}(1-b)\right\rangle$ \\
\hline$\rightarrow_{95}$ & $\begin{array}{l}\langle\min (b, \overline{s g}(c))+\operatorname{sg}(c)(\overline{s g}(1-b)+\min (c, \operatorname{sg}(1-b))), \\
\quad \min (1-b, \overline{s g}(c))+\min (1-c, \operatorname{sg}(c), \operatorname{sg}(1-b))\rangle\end{array}$ \\
\hline$\rightarrow_{96}$ & $\langle\max (\overline{s g}(1-c), b), \min (\operatorname{sg}(1-b), 1-c)\rangle$ \\
\hline$\rightarrow_{97}$ & $\langle\max (\overline{s g}(1-c), \operatorname{sg}(b)), \min (\operatorname{sg}(1-c), \overline{s g}(b))\rangle$ \\
\hline$\rightarrow_{98}$ & $\langle\max (\overline{s g}(1-c), b), 1-\max (b, c)\rangle$ \\
\hline$\rightarrow_{99}$ & $\langle\max (\overline{s g}(1-c), \overline{s g}(1-b)), \min (1-c, \overline{s g}(b))\rangle$ \\
\hline$\rightarrow_{100}$ & $\langle\max (b \operatorname{sg}(a), c), \min (a \operatorname{sgg}(b), d)\rangle$ \\
\hline$\rightarrow_{101}$ & $\langle\max (b \operatorname{sg}(a), c \operatorname{sg}(d)), \min (a \operatorname{sg}(b), \operatorname{sg}(c) d)\rangle$ \\
\hline$\rightarrow_{102}$ & $\langle\max (b, c \operatorname{sg}(d)), \min (a, \operatorname{sg}(c) d)\rangle$ \\
\hline$\rightarrow_{103}$ & $\langle\max (\min (1-a, \operatorname{sg}(a)), 1-d), \min (a, \operatorname{sg}(1-a), d)\rangle$ \\
\hline$\rightarrow_{104}$ & $\begin{array}{c}\langle\max (\min (1-a, \operatorname{sg}(a)), \min (1-d, \operatorname{sg}(d))) \\
\min (a, \operatorname{sg}(1-a), d, \operatorname{sg}(1-d))\rangle\end{array}$ \\
\hline$\rightarrow_{105}$ & $\langle\max (1-a, \min (1-d, \operatorname{sg}(d))), \min (a, d, \operatorname{sg}(1-d))\rangle$ \\
\hline$\rightarrow_{106}$ & $\langle\max (\min (b, \operatorname{sg}(1-b)), c), \min (1-b, \operatorname{sg}(b), 1-c)\rangle$ \\
\hline$\rightarrow_{107}$ & $\begin{array}{l}\langle\max (\min (b, \operatorname{sg}(1-b)), \min (c, \operatorname{sg}(1-c))) \\
\quad \min (1-b, \operatorname{sg}(b), 1-c, \operatorname{sg}(c))\rangle\end{array}$ \\
\hline$\rightarrow_{108}$ & $\langle\max (b, \min (c, \operatorname{sg}(1-c))), \min (1-b, 1-c, \operatorname{sg}(c))\rangle$ \\
\hline$\rightarrow_{109}$ & $\langle b+\min (\overline{s g}(1-a), c), a b+\min (\overline{s g}(1-a), d))\rangle$ \\
\hline$\rightarrow_{110}$ & $\langle\max (b, c), \min (a b+\overline{s g}(1-a), d)\rangle$ \\
\hline$\rightarrow_{111}$ & $\begin{array}{c}\langle\max (b, c d+\overline{s g}(1-c)), \min (a b+\overline{s g}(1-a) \\
\quad d(c d+\overline{s g}(1-c))+\overline{s g}(1-d))\rangle\end{array}$ \\
\hline$\rightarrow_{112}$ & $\langle b+c-b c, a b+\overline{s g}(1-a) d\rangle$ \\
\hline$\rightarrow_{113}$ & $\begin{array}{c}\langle b+c d-b(c d+\overline{s g}(1-c)) \\
(a b+\overline{s g}(1-a))(d(c d+\overline{s g}(1-c))+\overline{s g}(1-d))\rangle\end{array}$ \\
\hline$\rightarrow_{114}$ & $\langle 1-a+\min (\overline{s g}(1-a), 1-d), a(1-a)+\min (\overline{s g}(1-a), d)\rangle$ \\
\hline$\rightarrow_{115}$ & $\langle 1-\min (a, d), \min (a(1-a)+\overline{s g}(1-a), d)\rangle$ \\
\hline$\rightarrow_{116}$ & $\begin{array}{c}\langle\max (1-a,(1-d) d+\overline{s g}(d)) \\
\min (a(1-a)+\overline{s g}(1-a), d((1-d) d+\overline{s g}(d))+\overline{s g}(1-d))\rangle\end{array}$ \\
\hline$\rightarrow_{117}$ & $\langle 1-a-d+a d,(a(1-a)+\overline{s g}(1-a)) d\rangle$ \\
\hline$\rightarrow_{118}$ & $\begin{array}{c}\langle 1-a+(1-d) d-(1-a)((1-d) d+\overline{s g}(d)) \\
(a(1-a)+\overline{s g}(1-a)) d((1-d) d+\overline{s g}(d))+\overline{s g}(1-d)\rangle\end{array}$ \\
\hline$\rightarrow_{119}$ & $\langle b+\min (\overline{s g}(b), c),(1-b) b+\min (\overline{s g}(b), 1-c)\rangle$ \\
\hline$\rightarrow_{120}$ & $\langle\max (b, c), \min ((1-b) b+\overline{s g}(b), 1-c)\rangle$ \\
\hline$\rightarrow_{121}$ & $\begin{array}{c}\langle\max (b, c(1-c)+\overline{s g}(1-c)) \\
\min ((1-b) b+\overline{s g}(b),(1-c)(c(1-c)+\overline{s g}(1-c)))+\overline{s g}(c)\rangle\end{array}$ \\
\hline$\rightarrow_{122}$ & $\langle b+c-b c,((1-b) b+\overline{s g}(b))(1-c)\rangle$ \\
\hline$\rightarrow_{123}$ & $\langle b+c(1-c)-(b(c(1-c)+\overline{s g}(1-c)))$, \\
\hline
\end{tabular}

(Continued on next page) 
Table 1 (Continued from previous page)

\begin{tabular}{|c|c|}
\hline & $((1-b) b+\overline{s g}(b))(((1-c)(c(1-c)+\overline{s g}(1-c)))+\overline{s g}(c))\rangle$ \\
\hline$\rightarrow_{124}$ & $\langle c+\min (\overline{s g}(1-d), b), c d+\min (\overline{s g}(1-d), a)\rangle$ \\
\hline$\rightarrow_{125}$ & $\langle\max (b, c), \min (c d+\overline{s g}(1-d), a)\rangle$ \\
\hline$\rightarrow_{126}$ & $\begin{array}{c}\langle\max (c, a b+\overline{s g}(1-b)), \\
\min (c d+\overline{s g}(1-d), a(a b+\overline{s g}(1-b))+\overline{s g}(1-a))\rangle\end{array}$ \\
\hline$\rightarrow_{127}$ & $\langle b+c-b c,(c d+\overline{s g}(1-d)) a\rangle$ \\
\hline$\rightarrow_{128}$ & $\begin{array}{c}\langle c+a b-c(a b+\overline{s g}(1-b)) \\
(c d+\overline{s g}(1-d))(a(a b+\overline{s g}(1-b))+\overline{s g}(1-a))\rangle\end{array}$ \\
\hline$\rightarrow_{129}$ & $\langle 1-d+\min (\overline{s g}(1-d), 1-a), d(1-d)+\min (\overline{s g}(1-d), a)\rangle$ \\
\hline$\rightarrow_{130}$ & $\langle 1-\min (d, a), \min (d(1-d)+\overline{s g}(1-d), a)\rangle$ \\
\hline$\rightarrow_{131}$ & $\begin{array}{c}\langle\max (1-d,(1-a) a+\overline{s g}(a)) \\
\min (d(1-d)+\overline{s g}(1-d), a((1-a) a+\overline{s g}(a))+\overline{s g}(1-a))\rangle\end{array}$ \\
\hline$\rightarrow_{132}$ & $\langle 1-a d,(d(1-d)+\overline{s g}(1-d)) a\rangle$ \\
\hline$\rightarrow_{133}$ & $\begin{array}{c}\langle 1-d+(1-a) a-(1-d)((1-a) a+\overline{s g}(a)) \\
(d(1-d)+\overline{s g}(1-d))(a((1-a) a+\overline{s g}(a))+\overline{s g}(1-a))\rangle\end{array}$ \\
\hline$\rightarrow_{134}$ & $\langle c+\min (\overline{s g}(c), b),(1-c) c+\min (\overline{s g}(c),(1-b))\rangle$ \\
\hline$\rightarrow_{135}$ & $\langle\max (b, c), \min ((1-c) c+\overline{s g}(c), 1-b)\rangle$ \\
\hline$\rightarrow_{136}$ & $\begin{array}{c}\langle\max (c, b(1-b)+\overline{s g}(1-b)) \\
\min ((1-c) c+\overline{s g}(c),(1-b)(b(1-b)+\overline{s g}(1-b))+\overline{s g}(b))\rangle\end{array}$ \\
\hline$\rightarrow_{137}$ & $\langle b+c-b c,((1-c) c+\overline{s g}(c))(1-b)\rangle$ \\
\hline$\rightarrow_{138}$ & $\begin{array}{c}\langle c+b(1-b)-c(b(1-b)+\overline{s g}(1-b)) \\
((1-c) c+\overline{s g}(c))((1-b)(b(1-b)+\overline{s g}(1-b))+\overline{s g}(b))\rangle\end{array}$ \\
\hline$\rightarrow_{139}$ & $\left\langle\frac{b+c}{2}, \frac{a+d}{2}\right\rangle$ \\
\hline$\rightarrow_{140}$ & $\left\langle\frac{b+c+\min (b, c)}{3}, \frac{a+d+\max (a, d)}{3}\right\rangle$ \\
\hline$\rightarrow_{141}$ & $\left\langle\frac{b+c+\max (b, c)}{3}, \frac{a+d+\min (a, d)}{3}\right\rangle$ \\
\hline$\rightarrow_{142}$ & $\left\langle\frac{3-a-d-\max (a, d)}{3}, \frac{a+d+\max (a, d)}{3}\right\rangle$ \\
\hline$\rightarrow_{143}$ & $\left\langle\frac{1-a+c+\min (1-a, c)}{3}, \frac{2+a-c-\min (1-a, c)}{3}\right\rangle$ \\
\hline$\rightarrow_{144}$ & $\left\langle\frac{1+b-d+\min (b, 1-d)}{3}, \frac{2-b+d-\min (b, 1-d)}{3}\right\rangle$ \\
\hline$\rightarrow_{145}$ & $\left\langle\frac{b+c+\min (b, c)}{3}, \frac{3-b-c-\min (b, c)}{3}\right\rangle$ \\
\hline$\rightarrow_{146}$ & $\left\langle\frac{3-a-d-\min (a, d)}{3}, \frac{a+d+\min (a, d)}{3}\right\rangle$ \\
\hline$\rightarrow_{147}$ & $\left\langle\frac{1-a+c+\max (1-a, c)}{3}, \frac{2+a-c-\max (1-a, c)}{3}\right\rangle$ \\
\hline$\rightarrow_{148}$ & $\left\langle\frac{1+b-d+\max (b, 1-d)}{3}, \frac{2-b+d-\max (b, 1-d)}{3}\right\rangle$ \\
\hline$\rightarrow_{149}$ & $\left\langle\frac{b+c+\max (b, c)}{3}, \frac{3-b-c-\max (b, c)}{3}\right\rangle$ \\
\hline$\rightarrow_{150, \lambda}$ & $\left\langle\frac{b+c+\lambda-1}{2 \lambda}, \frac{a+d+\lambda-1}{2 \lambda}\right.$, where $\left.\lambda \geq 1\right\rangle$ \\
\hline$\rightarrow_{151, \gamma}$ & $\left\langle\frac{b+c+\gamma}{2 \gamma+1}, \frac{a+d+\gamma-1}{2 \gamma+1}\right.$, where $\left.\gamma \geq 1\right\rangle$ \\
\hline$\rightarrow_{152, \alpha, \beta}$ & $\left\langle\frac{b+c+\alpha-1}{\alpha+\beta}, \frac{a+d+\beta-1}{\alpha+\beta}\right.$ where $\alpha \geq 1, \beta \in[1, \alpha]$ \\
\hline$\rightarrow_{153, \varepsilon, \eta}$ & $\langle\min (1, \max (c, b+\varepsilon)), \max (0, \min (d, a-\eta))\rangle$ \\
\hline
\end{tabular}

(Continued on next page) 
Table 1 (Continued from previous page)

\begin{tabular}{|c|c|}
\hline & where $\varepsilon, \eta \in[0,1]$ and $\varepsilon \leq \eta<1$ \\
\hline$\rightarrow 154, \lambda$ & $\left\langle\frac{-a+c+\lambda}{2 \lambda}, \frac{a-c+\lambda}{2 \lambda}\right\rangle$, where $\lambda \geq 1$ \\
\hline$\rightarrow_{155, \lambda}$ & $\left\langle\frac{1-a-d+\lambda}{2 \lambda}, \frac{a+d+\lambda-1}{2 \lambda}\right\rangle$, where $\lambda \geq 1$ \\
\hline$\rightarrow_{156, \lambda}$ & $\left\langle\frac{b+c+\lambda-1}{2 \lambda}, \frac{1-b-c+\lambda}{2 \lambda}\right\rangle$, where $\lambda \geq 1$ \\
\hline$\rightarrow_{157, \lambda}$ & $\left\langle\frac{b-d+\lambda}{2 \lambda}, \frac{-b+d+\lambda}{2 \lambda}\right\rangle$, where $\lambda \geq 1$ \\
\hline$\rightarrow_{158, \gamma}$ & $\left\langle\frac{1-a+c+\gamma}{2 \gamma+1}, \frac{a-c+\gamma}{2 \gamma+1}\right\rangle$, where $\gamma \geq 1$ \\
\hline$\rightarrow_{159, \gamma}$ & $\left\langle\frac{2-a-d+\gamma}{2 \gamma+1}, \frac{a+d+\gamma-1}{2 \gamma+1}\right\rangle$, where $\gamma \geq 1$ \\
\hline$\rightarrow_{160, \gamma}$ & $\left\langle\frac{b-d+\gamma+1}{2 \gamma+1}, \frac{-b+d+\gamma}{2 \gamma+1}\right\rangle$, where $\gamma \geq 1$ \\
\hline$\rightarrow_{161, \gamma}$ & $\left\langle\frac{b+c+\gamma}{2 \gamma+1}, \frac{1-b-c+\gamma}{2 \gamma+1}\right\rangle$, where $\gamma \geq 1$ \\
\hline$\rightarrow_{162, \alpha, \beta}$ & $\left\langle\frac{-a+c+\alpha}{\alpha+\beta}, \frac{a-c+\beta}{\alpha+\beta}\right\rangle$, where $\alpha \geq 1, \beta \in[1, \alpha]$ \\
\hline$\rightarrow_{163, \alpha, \beta}$ & $\left\langle\frac{1-a-d+\alpha}{\alpha+\beta}, \frac{a+d+\beta-1}{\alpha+\beta}\right\rangle$, where $\alpha \geq 1, \beta \in[1, \alpha]$ \\
\hline$\rightarrow_{164, \alpha, \beta}$ & $\left\langle\frac{b-d+\alpha}{\alpha+\beta}, \frac{-b+d+\beta}{\alpha+\beta}\right\rangle$, where $\alpha \geq 1, \beta \in[1, \alpha]$ \\
\hline$\rightarrow_{165, \alpha, \beta}$ & $\left\langle\frac{b+c+\alpha-1}{\alpha+\beta}, \frac{1-b-c+\beta}{\alpha+\beta}\right\rangle$, where $\alpha \geq 1, \beta \in[1, \alpha]$ \\
\hline$\rightarrow_{166}$ & $\langle\max (b, \min (a, c)), \min (a, \max (b, d))\rangle$ \\
\hline$\rightarrow_{167}$ & $\langle\max (1-a, \min (a, c)), \min (a, 1-\min (a, c))\rangle$ \\
\hline$\rightarrow_{168}$ & $\langle\max (1-a, \min (a, 1-d)), 1-\max (1-a, \min (a, 1-d))\rangle$ \\
\hline$\rightarrow_{169}$ & $\langle\max (b, \min (1-b, c)), 1-\max (b, \min (1-b, c))\rangle$ \\
\hline$\rightarrow_{170}$ & $\langle\max (b, \min (1-b, 1-d)), 1-\max (b, \min (1-b, 1-d))\rangle$ \\
\hline$\rightarrow_{171}$ & $\langle\overline{s g}(\max (a, d)-\max (b, c)), \operatorname{sg}(\max (a, d)-\max (b, c))\rangle$ \\
\hline$\rightarrow_{172}$ & $\langle\overline{s g}(a-c), \operatorname{sg}(a-c)\rangle$ \\
\hline$\rightarrow_{173}$ & $\langle\overline{s g}(a+d-1), \operatorname{sg}(a+d-1)\rangle$ \\
\hline$\rightarrow_{174}$ & $\langle\overline{s g}(1-b-c), \operatorname{sg}(1-b-c)\rangle$ \\
\hline$\rightarrow_{175}$ & $\langle\overline{s g}(d-b), \operatorname{sg}(d-b)\rangle$ \\
\hline$\rightarrow_{176}$ & $\langle\overline{s g}(a-c)+\operatorname{sg}(a-c) \max (b, c), \operatorname{sg}(a-c) \min (a, d)\rangle$ \\
\hline$\rightarrow_{177}$ & $\langle\overline{s g}(a-c)+\operatorname{sg}(a-c) \max (1-a, c), \operatorname{sg}(a-c) \min (a, 1-c)\rangle$ \\
\hline$\rightarrow_{178}$ & $\begin{array}{c}\langle\overline{s g}(a-1+d)+\operatorname{sg}(a-1+d)(1-\min (a, d)) \\
\operatorname{sg}(a-1+d) \min (a, d)\rangle\end{array}$ \\
\hline$\rightarrow_{179}$ & $\begin{array}{c}\langle\overline{s g}(1-b-c)+\operatorname{sg}(1-b-c) \max (b, c) \\
\operatorname{sg}(1-b-c)(1-\max (b, c))\rangle\end{array}$ \\
\hline$\rightarrow_{180}$ & $\langle\overline{s g}(d-b)+\operatorname{sg}(d-b) \max (b, 1-d), \operatorname{sg}(d-b) \min (1-b, d)\rangle$ \\
\hline$\rightarrow_{181}$ & $\langle 1-\operatorname{sg}(a) .(1-c), d \cdot \operatorname{sg}(a)\rangle$ \\
\hline$\rightarrow_{182}$ & $\langle 1-\operatorname{sg}(a) \cdot(1-c),(1-c) \cdot \operatorname{sg}(a)\rangle$ \\
\hline$\rightarrow_{183}$ & $\langle 1-\operatorname{sg}(a) . d, d \cdot \operatorname{sg}(a)\rangle$ \\
\hline$\rightarrow_{184}$ & $\langle 1-\operatorname{sg}(1-b) \cdot d, d \cdot \operatorname{sg}(1-b)\rangle$ \\
\hline$\rightarrow_{185}$ & $\langle 1-\operatorname{sg}(1-b) .(1-c),(1-c) \cdot \operatorname{sg}(1-b)\rangle$ \\
\hline$\rightarrow_{186}$ & $\langle\overline{s g}(d-b)+\operatorname{sg}(d-b) \max (b, c), \operatorname{sg}(d-b) \min (a, d)\rangle$ \\
\hline$\rightarrow_{187}$ & $\langle\overline{s g}(d-b)+\operatorname{sg}(d-b) \cdot \max (1-d, b), \operatorname{sg}(d-b) \min (d, 1-b)\rangle$ \\
\hline
\end{tabular}

(Continued on next page) 
Table 1 (Continued from previous page)

\begin{tabular}{|c|c|}
\hline$\rightarrow_{188}$ & $\langle\overline{s g}(a-1+d)+\operatorname{sg}(a-1+d) \cdot(1-\min (a, d)), \operatorname{sg}(a-1+d) \min (a, d)\rangle$ \\
\hline$\rightarrow_{189}$ & $\langle\overline{s g}(1-b-c)+\operatorname{sg}(1-b-c) \cdot \max (b, c), \operatorname{sg}(1-b-c)(1-\max (b, c))\rangle$ \\
\hline$\rightarrow_{190}$ & $\langle\overline{s g}(d-b)+\operatorname{sg}(d-b) \cdot \max (b, 1-d), \operatorname{sg}(d-b) \min (1-b, d)\rangle$ \\
\hline$\rightarrow_{191}$ & $\left\langle\overline{s g}(a-c)+\operatorname{sg}(a-c) \frac{c}{1-b}, \operatorname{sg}(a-c) \frac{a-c}{1-b}\right\rangle$ \\
\hline$\rightarrow_{192}$ & $\langle\max (c, \min (b, d)), \min (d, \max (a, c))\rangle$ \\
\hline$\rightarrow_{193}$ & $\langle\max (c, \min (b, d)), 1-\max (c, \min (b, d))\rangle$ \\
\hline$\rightarrow_{194}$ & $\langle\max (c, 1-\max (d, \max (a, c)), \min (d, \max (a, c))\rangle$ \\
\hline$\rightarrow_{195}$ & $\langle 1-\min (d, \max (a, 1-d)), \min (d, \max (a, 1-d))\rangle$ \\
\hline$\rightarrow_{196}$ & $\langle\max (c, \min (b, 1-c)), 1-\max (c, \min (b, 1-c))\rangle$ \\
\hline$\rightarrow_{197}$ & $\langle\max (1-d, \min (b, d)), 1-\max (1-d, \min (b, d))\rangle$ \\
\hline$\rightarrow_{198}$ & \\
\hline
\end{tabular}

Now, using the program product IFSTool (see [1]) we found the relations between every two implications - see Tables 2, 3, ., 13.

Table 2. Relations between the intuitionistic fuzzy implications $\rightarrow_{1}, \ldots, \rightarrow_{16}$

\begin{tabular}{|l|llllllllllllllll|}
\hline & 1 & 2 & 3 & 4 & 5 & 6 & 7 & 8 & 9 & 10 & 11 & 12 & 13 & 14 & 15 & 16 \\
\hline 1 & $=$ & - & - & $<$ & $<$ & - & $>$ & - & - & - & - & - & $<$ & - & - & - \\
\hline 2 & - & $=$ & $<$ & - & - & - & - & - & - & - & $<$ & - & - & - & - & - \\
\hline 3 & - & $>$ & $=$ & - & - & - & - & - & - & - & $<$ & - & - & - & $>$ & $>$ \\
\hline 4 & $>$ & - & - & $=$ & $<$ & - & $>$ & - & - & $>$ & - & $>$ & $<$ & - & - & - \\
\hline 5 & $>$ & - & - & $>$ & $=$ & $>$ & $>$ & - & - & $>$ & - & $>$ & $>$ & - & - & - \\
\hline 6 & - & - & - & - & $<$ & $=$ & - & - & - & - & - & - & $<$ & - & - & - \\
\hline 7 & $<$ & - & - & $<$ & $<$ & - & $=$ & - & - & - & - & - & $<$ & - & - & - \\
\hline 8 & - & - & - & - & - & - & - & - & - & - & $<$ & - & - & - & $>$ & - \\
\hline 9 & - & - & - & - & - & - & - & - & - & - & - & - & - & - & - & - \\
\hline 10 & - & - & - & $<$ & $<$ & - & - & - & - & $=$ & - & - & $<$ & - & - & - \\
\hline 11 & - & $>$ & $>$ & - & - & - & - & $>$ & - & - & $=$ & - & - & $>$ & $>$ & $>$ \\
\hline 12 & - & - & - & $<$ & $<$ & - & - & - & - & - & - & $=$ & $<$ & - & - & - \\
\hline 13 & $>$ & - & - & $>$ & $<$ & $>$ & $>$ & - & - & $>$ & - & $>$ & $=$ & - & - & - \\
\hline 14 & - & - & - & - & - & - & - & - & - & - & $<$ & - & - & $=$ & $>$ & - \\
\hline 15 & - & - & $<$ & - & - & - & - & $<$ & - & - & $<$ & - & - & $<$ & $=$ & - \\
\hline 16 & - & - & $<$ & - & - & - & - & - & - & - & $<$ & - & - & - & - & $=$ \\
\hline 17 & $>$ & - & - & $>$ & - & - & $>$ & - & - & $>$ & - & $>$ & - & - & - & - \\
\hline 18 & - & - & - & $<$ & $<$ & - & - & - & - & - & - & $>$ & $<$ & - & - & - \\
\hline 19 & - & - & $<$ & $<$ & $<$ & - & - & - & - & - & $<$ & - & $<$ & $<$ & - & $<$ \\
\hline 20 & - & - & - & - & - & - & - & - & - & - & - & - & - & - & - & - \\
\hline 21 & - & - & - & - & - & - & - & - & - & - & - & - & - & - & - & - \\
\hline
\end{tabular}

(Continued on next page) 
Table 2 (Continued from previous page)

\begin{tabular}{|c|c|c|c|c|c|c|c|c|c|c|c|c|c|c|c|c|}
\hline & 1 & 2 & 3 & 4 & 5 & 6 & 7 & 8 & 9 & 10 & 11 & 12 & 13 & 14 & 15 & 16 \\
\hline 22 & - & - & - & - & - & - & - & - & - & - & - & $>$ & - & - & - & - \\
\hline 23 & - & - & - & - & - & - & - & - & - & - & - & - & - & $>$ & $>$ & - \\
\hline 24 & - & - & - & - & - & - & - & $<$ & - & - & $<$ & - & - & - & - & - \\
\hline 25 & - & - & - & $<$ & $<$ & - & - & - & - & - & - & - & $<$ & - & - & - \\
\hline 26 & - & - & $<$ & $<$ & $<$ & - & - & - & - & - & $<$ & - & $<$ & - & - & $<$ \\
\hline 27 & - & - & - & - & - & - & - & - & - & - & - & - & - & - & - & - \\
\hline 28 & - & - & - & $<$ & $<$ & - & - & - & - & - & - & - & $<$ & - & - & - \\
\hline 29 & - & - & - & - & - & - & - & - & - & - & - & - & - & - & - & - \\
\hline 30 & $>$ & - & - & - & - & - & $>$ & - & - & - & - & - & - & - & - & - \\
\hline 31 & - & $>$ & - & - & - & - & - & - & - & - & - & - & - & - & $>$ & - \\
\hline 32 & - & $>$ & $>$ & - & - & - & - & $>$ & - & - & $>$ & - & - & $>$ & $>$ & $>$ \\
\hline 33 & $>$ & - & - & $>$ & - & - & $>$ & - & - & $>$ & - & $>$ & - & - & - & $>$ \\
\hline 34 & $>$ & $>$ & $>$ & $>$ & $>$ & $>$ & $>$ & $>$ & - & $>$ & $>$ & $>$ & $>$ & $>$ & $>$ & $>$ \\
\hline 35 & - & - & - & - & - & - & - & - & - & - & - & - & - & - & - & - \\
\hline 36 & - & - & - & - & - & - & - & - & - & - & - & - & - & - & - & - \\
\hline 37 & - & - & - & - & - & - & - & $>$ & - & - & - & - & - & - & $>$ & - \\
\hline 38 & - & - & - & - & - & - & - & - & - & - & - & - & - & - & - & - \\
\hline 39 & - & - & - & - & - & - & - & - & - & $>$ & - & - & - & - & - & - \\
\hline 40 & - & - & - & - & - & - & - & - & - & - & - & - & - & - & $>$ & - \\
\hline 41 & - & - & - & - & - & - & - & - & - & - & - & - & - & - & - & $>$ \\
\hline 42 & - & $>$ & $>$ & - & - & - & - & $>$ & - & - & $>$ & - & - & $>$ & $>$ & $>$ \\
\hline 43 & - & - & - & - & - & - & - & - & - & - & - & - & - & - & - & $>$ \\
\hline 44 & - & - & - & - & - & - & - & - & - & - & - & - & - & - & - & $>$ \\
\hline 45 & - & - & - & - & - & - & - & - & - & - & - & - & - & - & - & - \\
\hline 46 & - & - & - & $<$ & $<$ & - & - & - & - & - & - & $<$ & $<$ & - & - & - \\
\hline 47 & - & $<$ & $<$ & - & $<$ & - & - & - & - & - & $<$ & - & - & $<$ & - & - \\
\hline 48 & - & - & $<$ & - & $<$ & - & - & - & - & - & $<$ & - & - & $<$ & - & - \\
\hline 49 & - & - & - & - & $<$ & - & - & - & - & - & - & $>$ & - & - & - & - \\
\hline 50 & - & - & - & - & $<$ & - & - & - & - & - & - & $>$ & $<$ & - & - & - \\
\hline 51 & - & - & - & $<$ & $<$ & - & - & - & - & - & - & $<$ & $<$ & - & - & - \\
\hline 52 & - & - & $<$ & - & $<$ & - & - & $<$ & - & - & $<$ & - & - & $<$ & - & - \\
\hline 53 & - & - & - & - & $<$ & - & - & - & - & - & - & - & $<$ & - & - & - \\
\hline 54 & - & - & - & $<$ & $<$ & - & - & - & - & - & - & $<$ & $<$ & - & - & - \\
\hline 55 & - & $<$ & $<$ & - & $<$ & - & - & $<$ & - & - & $<$ & - & - & $<$ & $<$ & - \\
\hline 56 & - & - & $<$ & $<$ & $<$ & - & - & - & - & - & $<$ & $<$ & $<$ & $<$ & - & $<$ \\
\hline 57 & - & - & - & - & - & - & - & - & - & - & - & - & - & - & - & - \\
\hline 58 & - & - & - & $<$ & $<$ & - & - & - & - & - & - & $<$ & $<$ & - & - & - \\
\hline
\end{tabular}


Table 2 (Continued from previous page)

\begin{tabular}{|c|c|c|c|c|c|c|c|c|c|c|c|c|c|c|c|c|}
\hline & 1 & 2 & 3 & 4 & 5 & 6 & 7 & 8 & 9 & 10 & 11 & 12 & 13 & 14 & 15 & 16 \\
\hline 59 & - & - & - & $<$ & $<$ & - & - & - & - & - & - & $<$ & $<$ & - & - & - \\
\hline 60 & - & - & - & - & - & - & - & - & - & - & - & - & - & - & - & - \\
\hline 61 & - & - & - & $<$ & $<$ & - & $>$ & - & - & - & - & - & $<$ & - & - & - \\
\hline 62 & - & - & - & - & - & - & - & - & - & - & - & - & - & - & - & - \\
\hline 63 & - & - & - & - & - & - & - & - & - & - & - & - & - & - & $>$ & - \\
\hline 64 & - & - & - & - & $<$ & - & - & - & - & - & - & - & $<$ & - & - & - \\
\hline 65 & - & - & - & - & - & - & - & - & - & - & - & - & - & - & $>$ & - \\
\hline 66 & - & - & - & - & - & - & - & - & - & - & - & - & - & - & - & - \\
\hline 67 & - & - & - & $<$ & $<$ & - & - & - & - & - & - & - & $<$ & - & - & - \\
\hline 68 & - & - & - & - & - & - & - & - & - & - & - & - & - & - & $>$ & - \\
\hline 69 & - & - & - & - & - & - & - & - & - & - & - & - & - & - & $>$ & - \\
\hline 70 & - & - & - & - & - & - & - & - & - & - & - & - & - & - & - & - \\
\hline 71 & $>$ & - & - & $>$ & - & - & $>$ & - & - & $>$ & - & $>$ & - & - & - & - \\
\hline 72 & - & - & - & $<$ & $<$ & - & - & - & - & - & - & $>$ & $<$ & - & - & - \\
\hline 73 & - & - & - & $<$ & $<$ & - & - & - & - & $<$ & - & - & $<$ & - & - & - \\
\hline 74 & - & - & - & - & - & - & - & - & - & - & - & - & - & - & - & - \\
\hline 75 & - & - & - & - & - & - & - & - & - & - & - & - & - & - & - & - \\
\hline 76 & - & - & - & - & - & - & - & - & - & - & - & $>$ & - & - & - & - \\
\hline 77 & - & - & - & - & - & - & - & - & - & - & - & - & - & - & $>$ & - \\
\hline 78 & - & - & - & $<$ & $<$ & - & - & - & - & - & - & - & $<$ & - & - & - \\
\hline 79 & - & - & - & - & - & - & - & - & - & - & - & - & - & - & - & - \\
\hline 80 & - & - & - & $<$ & $<$ & - & - & - & - & - & - & - & $<$ & - & - & - \\
\hline 81 & - & - & - & - & - & - & - & - & - & - & - & - & - & - & - & - \\
\hline 82 & - & - & - & - & - & - & $>$ & - & - & - & - & - & - & - & - & - \\
\hline 83 & - & - & - & - & - & - & - & - & - & - & - & - & - & - & $>$ & - \\
\hline 84 & - & - & - & - & - & - & - & $>$ & - & - & - & - & - & - & $>$ & - \\
\hline 85 & - & - & - & - & - & - & - & - & - & - & - & - & - & - & - & - \\
\hline 86 & - & - & - & - & - & - & - & - & - & - & - & - & - & - & - & $>$ \\
\hline 87 & - & - & - & - & - & - & - & - & - & - & - & - & - & - & - & - \\
\hline 88 & - & - & - & - & - & - & - & $>$ & - & - & - & - & - & - & $>$ & - \\
\hline 89 & - & - & - & - & - & - & - & - & - & - & - & - & - & - & - & - \\
\hline 90 & - & - & - & - & - & - & - & - & - & - & - & - & - & - & - & - \\
\hline 91 & - & - & - & $<$ & $<$ & - & - & - & - & - & - & $<$ & $<$ & - & - & - \\
\hline 92 & - & - & - & - & $<$ & - & - & - & - & - & - & - & - & - & - & - \\
\hline 93 & - & - & - & - & $<$ & - & - & - & - & - & - & - & - & - & - & - \\
\hline 94 & - & - & - & - & $<$ & - & - & - & - & - & - & - & $<$ & - & - & - \\
\hline 95 & - & - & - & $<$ & $<$ & - & - & - & - & - & - & $<$ & $<$ & - & - & - \\
\hline
\end{tabular}


Table 2 (Continued from previous page)

\begin{tabular}{|c|c|c|c|c|c|c|c|c|c|c|c|c|c|c|c|c|}
\hline & 1 & 2 & 3 & 4 & 5 & 6 & 7 & 8 & 9 & 10 & 11 & 12 & 13 & 14 & 15 & 16 \\
\hline 96 & - & - & - & $<$ & $<$ & - & - & - & - & $<$ & - & $<$ & $<$ & - & - & - \\
\hline 97 & - & - & - & - & - & - & - & - & - & - & - & - & - & - & - & - \\
\hline 98 & - & - & - & $<$ & $<$ & - & - & - & - & - & - & $<$ & $<$ & - & - & - \\
\hline 99 & - & - & - & - & - & - & - & - & - & - & - & - & - & - & - & - \\
\hline 100 & - & - & - & - & - & - & - & - & - & - & - & - & - & - & - & - \\
\hline 101 & - & - & - & - & - & - & - & - & - & - & - & - & - & - & - & - \\
\hline 102 & - & - & - & - & - & - & - & - & - & - & - & - & - & - & - & - \\
\hline 103 & - & - & - & - & - & - & - & - & - & - & - & - & - & - & - & - \\
\hline 104 & - & - & - & - & - & - & - & - & - & - & - & - & - & - & - & - \\
\hline 105 & - & - & - & - & - & - & - & - & - & - & - & - & - & - & - & - \\
\hline 106 & - & - & - & - & - & - & - & - & - & - & - & - & - & - & - & - \\
\hline 107 & - & - & - & - & - & - & - & - & - & - & - & - & - & - & - & - \\
\hline 108 & - & - & - & - & - & - & - & - & - & - & - & - & - & - & - & - \\
\hline 109 & - & - & - & - & - & - & - & - & - & - & - & - & - & - & - & - \\
\hline 110 & $>$ & - & - & $>$ & - & - & $>$ & - & - & $>$ & - & $>$ & - & - & - & - \\
\hline 111 & - & - & - & - & - & - & - & - & - & - & - & - & - & - & - & - \\
\hline 112 & $>$ & - & - & $>$ & - & $>$ & $>$ & - & $>$ & $>$ & - & $>$ & $>$ & - & - & - \\
\hline 113 & - & - & - & - & - & - & - & - & - & - & - & - & - & - & - & - \\
\hline 114 & - & - & - & - & - & - & - & - & - & - & - & - & - & - & - & - \\
\hline 115 & $>$ & - & - & $>$ & - & - & $>$ & - & - & $>$ & - & $>$ & - & - & - & $>$ \\
\hline 116 & - & - & - & - & - & - & - & - & - & - & - & - & - & - & - & - \\
\hline 117 & - & - & - & - & - & - & - & - & - & - & - & - & - & - & - & - \\
\hline 118 & - & - & - & - & - & - & - & - & - & - & - & - & - & - & - & - \\
\hline 119 & - & - & - & - & - & - & - & - & - & - & - & - & - & - & - & - \\
\hline 120 & - & - & - & - & - & - & - & - & - & - & - & $>$ & - & - & - & - \\
\hline 121 & - & - & - & - & - & - & - & - & - & - & - & - & - & - & - & - \\
\hline 122 & - & - & - & - & - & - & - & - & - & - & - & $>$ & - & - & - & - \\
\hline 123 & - & - & - & - & - & - & - & - & - & - & - & - & - & - & - & - \\
\hline 124 & - & - & - & - & - & - & - & - & - & - & - & - & - & - & - & - \\
\hline 125 & $>$ & - & - & $>$ & - & - & $>$ & - & - & $>$ & - & $>$ & - & - & - & - \\
\hline 126 & - & - & - & - & - & - & - & - & - & - & - & - & - & - & - & - \\
\hline 127 & $>$ & - & - & $>$ & - & $>$ & $>$ & - & - & $>$ & - & $>$ & $>$ & - & - & - \\
\hline 128 & - & - & - & - & - & - & - & - & - & - & - & - & - & - & - & - \\
\hline 129 & - & - & - & - & - & - & - & - & - & - & - & - & - & - & - & - \\
\hline 130 & $>$ & - & - & $>$ & - & - & $>$ & - & - & $>$ & - & $>$ & - & - & - & $>$ \\
\hline 131 & - & - & - & - & - & - & - & - & - & - & - & - & - & - & - & $>$ \\
\hline 132 & - & - & - & - & - & - & - & - & - & - & - & - & - & - & - & - \\
\hline
\end{tabular}

(Continued on next page) 
Table 2 (Continued from previous page)

\begin{tabular}{|c|c|c|c|c|c|c|c|c|c|c|c|c|c|c|c|c|}
\hline & 1 & 2 & 3 & 4 & 5 & 6 & 7 & 8 & 9 & 10 & 11 & 12 & 13 & 14 & 15 & 16 \\
\hline 133 & - & - & - & - & - & - & - & - & - & - & - & - & - & - & - & - \\
\hline 134 & - & - & - & - & - & - & - & - & - & - & - & - & - & - & - & - \\
\hline 135 & - & - & - & - & - & - & - & - & - & - & - & $>$ & - & - & - & - \\
\hline 136 & - & - & - & - & - & - & - & - & - & - & - & - & - & - & - & - \\
\hline 137 & - & - & - & - & - & - & - & - & - & - & - & $>$ & - & - & - & - \\
\hline 138 & - & - & - & - & - & - & - & - & - & - & - & - & - & - & - & - \\
\hline 139 & - & - & - & $<$ & $<$ & - & - & - & - & - & - & - & $<$ & - & - & - \\
\hline 140 & - & - & - & $<$ & $<$ & - & - & - & - & - & - & - & $<$ & - & - & - \\
\hline 141 & - & - & - & $<$ & $<$ & - & - & - & - & - & - & - & $<$ & - & - & - \\
\hline 142 & - & - & - & - & - & - & - & - & - & - & - & - & - & - & - & - \\
\hline 143 & - & - & - & - & - & - & - & - & - & - & - & - & - & - & - & - \\
\hline 144 & - & - & - & - & - & - & - & - & - & - & - & - & - & - & - & - \\
\hline 145 & - & - & - & $<$ & $<$ & - & - & - & - & - & - & $<$ & $<$ & - & - & - \\
\hline 146 & - & - & - & - & - & - & - & - & - & - & - & - & - & - & - & - \\
\hline 147 & - & - & - & - & - & - & - & - & - & - & - & - & - & - & - & - \\
\hline 148 & - & - & - & - & - & - & - & - & - & - & - & - & - & - & - & - \\
\hline 149 & - & - & - & $<$ & $<$ & - & - & - & - & - & - & $<$ & $<$ & - & - & - \\
\hline 166 & $<$ & - & - & $<$ & $<$ & - & $>$ & - & - & - & - & - & $<$ & - & - & - \\
\hline 167 & - & - & - & - & - & - & - & - & - & - & - & - & - & - & - & - \\
\hline 168 & - & - & - & - & - & - & - & - & - & - & - & - & - & - & - & - \\
\hline 169 & - & - & - & $<$ & $<$ & - & - & - & - & - & - & $<$ & $<$ & - & - & - \\
\hline 170 & - & - & - & - & - & - & - & - & - & - & - & - & - & - & - & - \\
\hline 171 & - & - & - & - & - & - & - & - & - & - & - & - & - & - & - & - \\
\hline 172 & - & $<$ & $<$ & - & - & - & - & $<$ & - & - & $<$ & - & - & - & - & - \\
\hline 173 & - & - & - & - & - & - & - & - & - & - & - & - & - & - & $>$ & - \\
\hline 174 & - & $<$ & $<$ & - & $<$ & - & - & $<$ & - & - & $<$ & - & - & $<$ & $<$ & - \\
\hline 175 & - & - & - & - & - & - & - & - & - & - & - & - & - & - & - & - \\
\hline 176 & $>$ & $>$ & $>$ & $>$ & - & - & $>$ & - & - & $>$ & - & $>$ & - & - & $>$ & $>$ \\
\hline 177 & - & - & - & - & - & - & - & - & - & - & - & $>$ & - & - & $>$ & - \\
\hline 178 & $>$ & $>$ & $>$ & $>$ & - & - & $>$ & $>$ & - & $>$ & $>$ & $>$ & - & $>$ & $>$ & $>$ \\
\hline 179 & - & - & - & - & $<$ & - & - & - & - & - & - & $>$ & - & - & - & - \\
\hline 180 & - & - & - & - & - & - & - & - & - & - & - & $>$ & - & $>$ & $>$ & - \\
\hline 181 & - & - & $<$ & - & - & - & - & - & - & - & $<$ & - & - & - & - & $"="$ \\
\hline 182 & - & - & $<$ & - & - & - & - & - & - & - & $<$ & - & - & - & - & $<$ \\
\hline 183 & - & - & - & - & - & - & - & - & - & - & - & - & - & - & - & $>$ \\
\hline 184 & - & - & - & - & - & - & - & - & - & - & - & - & - & - & - & - \\
\hline 185 & - & - & $<$ & $<$ & $<$ & - & - & - & - & - & $<$ & $<$ & $<$ & $<$ & - & $<$ \\
\hline
\end{tabular}


Table 2 (Continued from previous page)

\begin{tabular}{|c|cccccccccccccccc|}
\hline & 1 & 2 & 3 & 4 & 5 & 6 & 7 & 8 & 9 & 10 & 11 & 12 & 13 & 14 & 15 & 16 \\
\hline 186 & $>$ & - & - & $>$ & - & - & $>$ & - & - & $>$ & - & $>$ & - & - & $>$ & - \\
\hline 187 & - & - & - & - & - & - & - & - & - & - & - & $>$ & - & $>$ & $>$ & - \\
\hline 188 & $>$ & $>$ & $>$ & $>$ & - & - & $>$ & $>$ & - & $>$ & $>$ & $>$ & - & $>$ & $>$ & $>$ \\
\hline 189 & - & - & - & - & $<$ & - & - & - & - & - & - & $>$ & - & - & - & - \\
\hline 190 & - & - & - & - & - & - & - & - & - & - & - & $>$ & - & $>$ & $>$ & - \\
\hline 191 & - & - & - & - & - & - & - & - & - & - & - & - & - & - & - & - \\
\hline 192 & - & - & - & $<$ & $<$ & - & $>$ & - & - & - & - & - & $<$ & - & - & - \\
\hline 193 & - & - & - & $<$ & $<$ & - & - & - & - & - & - & $<$ & $<$ & - & - & - \\
\hline 194 & - & - & - & - & - & - & $>$ & - & - & - & - & - & - & - & - & - \\
\hline 195 & - & - & - & - & - & - & - & - & - & - & - & - & - & - & - & - \\
\hline 196 & - & - & - & - & - & - & - & - & - & - & - & - & - & - & - & - \\
\hline 197 & - & - & - & $<$ & $<$ & - & - & - & - & - & - & $<$ & $<$ & - & - & - \\
\hline 198 & - & - & - & - & - & - & - & - & - & - & - & - & - & - & - & - \\
\hline
\end{tabular}

Table 3. Relations between the intuitionistic fuzzy implications $\rightarrow_{17}, \ldots, \rightarrow_{32}$

\begin{tabular}{|l|llllllllllllllll|}
\hline & 17 & 18 & 19 & 20 & 21 & 22 & 23 & 24 & 25 & 26 & 27 & 28 & 29 & 30 & 31 & 32 \\
\hline 1 & $<$ & - & - & - & - & - & - & - & - & - & - & - & - & $<$ & - & - \\
\hline 2 & - & - & - & - & - & - & - & - & - & - & - & - & - & - & $<$ & $<$ \\
\hline 3 & - & - & $>$ & - & - & - & - & - & - & $>$ & - & - & - & - & - & $<$ \\
\hline 4 & $<$ & $>$ & $>$ & - & - & - & - & - & $>$ & $>$ & - & $>$ & - & - & - & - \\
\hline 5 & - & $>$ & $>$ & - & - & - & - & - & $>$ & $>$ & - & $>$ & - & - & - & - \\
\hline 6 & - & - & - & - & - & - & - & - & - & - & - & - & - & - & - & - \\
\hline 7 & $<$ & - & - & - & - & - & - & - & - & - & - & - & - & $<$ & - & - \\
\hline 8 & - & - & - & - & - & - & - & $>$ & - & - & - & - & - & - & - & $<$ \\
\hline 9 & - & - & - & - & - & - & - & - & - & - & - & - & - & - & - & - \\
\hline 10 & $<$ & - & - & - & - & - & - & - & - & - & - & - & - & - & - & - \\
\hline 11 & - & - & $>$ & - & - & - & - & $>$ & - & $>$ & - & - & - & - & - & $<$ \\
\hline 12 & $<$ & $<$ & - & - & - & $<$ & - & - & - & - & - & - & - & - & - & - \\
\hline 13 & - & $>$ & $>$ & - & - & - & - & - & $>$ & $>$ & - & $>$ & - & - & - & - \\
\hline 14 & - & - & $>$ & - & - & - & $<$ & - & - & - & - & - & - & - & - & $<$ \\
\hline 15 & - & - & - & - & - & - & $<$ & - & - & - & - & - & - & - & $<$ & $<$ \\
\hline 16 & - & - & $>$ & - & - & - & - & - & - & $>$ & - & - & - & - & - & $<$ \\
\hline 17 & $=$ & $>$ & $>$ & - & - & - & - & - & $>$ & $>$ & - & $>$ & - & - & - & - \\
\hline 18 & $<$ & $=$ & $>$ & - & - & $<$ & - & - & - & - & - & - & - & - & - & - \\
\hline 19 & $<$ & $<$ & - & - & - & $<$ & $<$ & - & - & $<$ & $<$ & $<$ & - & - & - & $<$ \\
\hline 20 & - & - & - & - & - & - & - & - & - & - & - & - & - & - & - & - \\
\hline 21 & - & - & - & - & - & - & - & - & $>$ & - & - & - & - & - & - & - \\
\hline & & & & & $(C o n t i n u e d$ & $0 n$ & $n e x t$ & $p a g e$ & & & & & & \\
\hline
\end{tabular}


Table 3 (Continued from previous page)

\begin{tabular}{|c|c|c|c|c|c|c|c|c|c|c|c|c|c|c|c|c|}
\hline & 17 & 18 & 19 & 20 & 21 & 22 & 23 & 24 & 25 & 26 & 27 & 28 & 29 & 30 & 31 & 32 \\
\hline 22 & - & $>$ & $>$ & - & - & $=$ & - & - & - & - & - & - & - & - & - & - \\
\hline 23 & - & - & $>$ & - & - & - & $=$ & - & - & - & - & - & - & - & - & - \\
\hline 24 & - & - & - & - & - & - & - & $=$ & - & - & - & - & - & - & $<$ & $<$ \\
\hline 25 & $<$ & - & - & - & $<$ & - & - & - & $=$ & - & - & $<$ & $<$ & - & - & - \\
\hline 26 & $<$ & - & $>$ & - & - & - & - & - & - & $=$ & $<$ & $<$ & - & - & - & $<$ \\
\hline 27 & - & - & $>$ & - & - & - & - & - & - & $>$ & $=$ & - & - & - & - & - \\
\hline 28 & $<$ & - & $>$ & - & - & - & - & - & $>$ & $>$ & - & $=$ & - & - & - & - \\
\hline 29 & - & - & - & - & - & - & - & - & $>$ & - & - & - & $=$ & - & - & - \\
\hline 30 & - & - & - & - & - & - & - & - & - & - & - & - & - & $=$ & - & - \\
\hline 31 & - & - & - & - & - & - & - & $>$ & - & - & - & - & - & - & $=$ & $<$ \\
\hline 32 & - & - & $>$ & - & - & - & - & $>$ & - & $>$ & - & - & - & - & $>$ & $=$ \\
\hline 33 & - & $>$ & $>$ & - & - & $>$ & - & - & $>$ & $>$ & - & $>$ & - & $>$ & - & - \\
\hline 34 & - & $>$ & $>$ & - & - & $>$ & - & $>$ & $>$ & $>$ & - & $>$ & - & $>$ & $>$ & $>$ \\
\hline 35 & - & - & - & - & - & - & - & - & - & - & - & - & - & - & - & - \\
\hline 36 & - & - & - & - & - & - & - & - & - & - & - & - & - & $<$ & - & - \\
\hline 37 & - & - & - & - & - & - & - & $>$ & - & - & - & - & - & - & - & $<$ \\
\hline 38 & - & - & - & - & - & - & - & - & - & - & - & - & - & - & - & - \\
\hline 39 & - & - & - & - & - & - & - & - & - & - & - & - & - & - & - & - \\
\hline 40 & - & - & - & - & - & - & - & $>$ & - & - & - & - & - & - & $<$ & $<$ \\
\hline 41 & - & - & $>$ & - & - & - & - & - & - & $>$ & - & - & - & - & - & $<$ \\
\hline 42 & - & - & $>$ & $>$ & - & - & $>$ & $>$ & - & $>$ & $>$ & - & - & - & $>$ & $>$ \\
\hline 43 & - & - & $>$ & - & - & - & - & - & $>$ & $>$ & - & $>$ & - & - & - & - \\
\hline 44 & - & - & $>$ & - & - & - & - & - & $>$ & $>$ & - & $>$ & - & - & - & - \\
\hline 45 & - & - & - & - & - & - & - & - & $>$ & - & - & - & $>$ & - & - & - \\
\hline 46 & $<$ & $<$ & - & - & - & $<$ & - & - & - & - & - & - & - & - & - & - \\
\hline 47 & - & - & - & $<$ & - & - & $<$ & - & - & - & $<$ & - & - & - & $<$ & $<$ \\
\hline 48 & - & - & - & $<$ & - & - & $<$ & - & - & - & $<$ & - & - & - & - & $<$ \\
\hline 49 & - & - & - & - & - & - & - & - & - & - & - & - & - & - & - & - \\
\hline 50 & - & - & - & - & - & - & - & - & - & - & - & - & - & - & - & - \\
\hline 51 & $<$ & $<$ & - & - & - & $<$ & - & - & - & - & - & - & - & - & - & - \\
\hline 52 & - & - & - & $<$ & - & - & $<$ & - & - & - & $<$ & - & - & - & - & $<$ \\
\hline 53 & - & - & - & - & - & - & - & - & - & - & - & - & - & - & - & - \\
\hline 54 & $<$ & $<$ & - & - & - & $<$ & - & - & - & - & - & - & - & - & - & - \\
\hline 55 & - & - & - & $<$ & - & - & $<$ & $<$ & - & - & $<$ & - & - & - & $<$ & $<$ \\
\hline 56 & $<$ & $<$ & $<$ & $<$ & - & $<$ & $<$ & - & - & $<$ & $<$ & $<$ & - & - & - & $<$ \\
\hline 57 & - & - & - & $<$ & - & - & $<$ & - & - & - & $<$ & - & - & - & - & - \\
\hline 58 & $<$ & $<$ & - & - & $z^{2}$ & $<$ & - & - & $<$ & - & - & $<$ & $<$ & - & - & - \\
\hline
\end{tabular}


Table 3 (Continued from previous page)

\begin{tabular}{|c|c|c|c|c|c|c|c|c|c|c|c|c|c|c|c|c|}
\hline & 17 & 18 & 19 & 20 & 21 & 22 & 23 & 24 & 25 & 26 & 27 & 28 & 29 & 30 & 31 & 32 \\
\hline 59 & $<$ & $<$ & - & - & - & $<$ & - & - & - & - & - & $<$ & - & - & - & - \\
\hline 60 & - & - & - & - & - & - & - & - & - & - & - & - & $<$ & - & - & - \\
\hline 61 & $<$ & - & - & - & - & - & - & - & - & - & - & - & - & - & - & - \\
\hline 62 & - & - & - & - & - & - & - & - & - & - & - & - & - & - & - & - \\
\hline 63 & - & - & - & - & - & - & - & - & - & - & - & - & - & - & - & - \\
\hline 64 & - & - & - & - & - & - & - & - & - & - & - & - & - & - & - & - \\
\hline 65 & - & - & - & - & - & - & - & $>$ & - & - & - & - & - & - & - & $<$ \\
\hline 66 & - & - & - & - & - & - & - & - & - & - & - & - & - & - & - & - \\
\hline 67 & $<$ & - & $>$ & - & - & - & - & - & - & - & - & - & - & - & - & - \\
\hline 68 & - & - & - & - & - & - & - & $>$ & - & - & - & - & - & - & - & - \\
\hline 69 & - & - & - & - & - & - & - & - & - & - & - & - & - & - & - & - \\
\hline 70 & - & - & - & - & - & - & - & - & - & - & - & - & - & - & - & - \\
\hline 71 & - & $>$ & $>$ & - & - & - & - & - & $>$ & $>$ & - & $>$ & - & - & - & - \\
\hline 72 & $<$ & - & - & - & - & - & - & - & - & - & - & - & - & - & - & - \\
\hline 73 & $<$ & - & - & - & $<$ & - & - & - & - & - & - & - & - & - & - & - \\
\hline 74 & - & - & - & - & - & - & - & - & - & - & - & - & - & - & - & - \\
\hline 75 & - & - & $>$ & - & - & - & - & - & $>$ & $>$ & - & $>$ & - & - & - & - \\
\hline 76 & - & - & - & - & - & - & - & - & - & - & - & - & - & - & - & - \\
\hline 77 & - & - & - & - & - & - & - & - & - & - & - & - & - & - & - & - \\
\hline 78 & $<$ & - & - & - & $<$ & - & - & - & - & - & - & - & - & - & - & - \\
\hline 79 & - & - & - & - & - & - & - & - & - & - & - & - & - & - & - & - \\
\hline 80 & $<$ & - & - & - & $<$ & - & - & - & $>$ & - & - & - & - & - & - & - \\
\hline 81 & - & - & - & - & - & - & - & - & $>$ & - & - & - & - & - & - & - \\
\hline 82 & - & - & - & - & - & - & - & - & - & - & - & - & - & - & - & - \\
\hline 83 & - & - & - & - & - & - & - & $>$ & - & - & - & - & - & - & - & - \\
\hline 84 & - & - & - & - & - & - & - & $>$ & - & - & - & - & - & - & - & - \\
\hline 85 & - & - & - & - & - & - & - & - & - & - & - & - & - & - & - & - \\
\hline 86 & - & - & $>$ & - & - & - & - & - & - & $>$ & - & - & - & - & - & - \\
\hline 87 & - & - & - & - & - & - & - & - & - & - & - & - & - & - & - & - \\
\hline 88 & - & - & - & - & - & - & - & $>$ & - & - & - & - & - & - & - & - \\
\hline 89 & - & - & - & - & - & - & - & - & $>$ & - & - & - & - & - & - & - \\
\hline 90 & - & - & - & - & - & - & - & - & $>$ & - & - & - & - & - & - & - \\
\hline 91 & $<$ & $<$ & - & - & - & $<$ & - & - & - & - & - & - & - & - & - & - \\
\hline 92 & - & - & - & - & - & - & - & - & - & - & - & - & - & - & - & - \\
\hline 93 & - & - & - & - & - & - & - & - & - & - & - & - & - & - & - & - \\
\hline 94 & - & - & - & - & - & - & - & - & - & - & - & - & - & - & - & - \\
\hline 95 & $<$ & $<$ & - & - & - & $<$ & - & - & - & - & - & - & - & - & - & - \\
\hline
\end{tabular}


Table 3 (Continued from previous page)

\begin{tabular}{|c|c|c|c|c|c|c|c|c|c|c|c|c|c|c|c|c|}
\hline & 17 & 18 & 19 & 20 & 21 & 22 & 23 & 24 & 25 & 26 & 27 & 28 & 29 & 30 & 31 & 32 \\
\hline 96 & $<$ & $<$ & - & - & $<$ & $<$ & - & - & - & - & - & - & - & - & - & - \\
\hline 97 & - & - & - & - & - & - & - & - & - & - & - & - & - & - & - & - \\
\hline 98 & $<$ & $<$ & - & - & $<$ & $<$ & - & - & - & - & - & - & - & - & - & - \\
\hline 99 & - & - & - & - & - & - & - & - & - & - & - & - & - & - & - & - \\
\hline 100 & - & - & - & - & - & - & - & - & - & - & - & - & - & - & - & - \\
\hline 101 & - & - & - & - & - & - & - & - & - & - & - & - & - & - & - & - \\
\hline 102 & - & - & - & - & - & - & - & - & - & - & - & - & - & - & - & - \\
\hline 103 & - & - & - & - & - & - & - & - & - & - & - & - & - & - & - & - \\
\hline 104 & - & - & - & - & - & - & - & - & - & - & - & - & - & - & - & - \\
\hline 105 & - & - & - & - & - & - & - & - & - & - & - & - & - & - & - & - \\
\hline 106 & - & - & - & - & - & - & - & - & - & - & - & - & - & - & - & - \\
\hline 107 & - & - & - & - & - & - & - & - & - & - & - & - & - & - & - & - \\
\hline 108 & - & - & - & - & - & - & - & - & - & - & - & - & - & - & - & - \\
\hline 109 & - & - & - & - & - & - & - & - & - & - & - & - & - & - & - & - \\
\hline 110 & $>$ & $>$ & $>$ & - & - & - & - & - & $>$ & $>$ & - & $>$ & - & - & - & - \\
\hline 111 & - & - & - & - & - & - & - & - & $>$ & - & - & - & - & - & - & - \\
\hline 112 & $>$ & $>$ & $>$ & - & - & - & - & - & $>$ & $>$ & - & $>$ & - & - & - & - \\
\hline 113 & - & - & - & - & - & - & - & - & - & - & - & - & - & - & - & - \\
\hline 114 & - & - & - & - & - & - & - & - & - & - & - & - & - & - & - & - \\
\hline 115 & - & $>$ & $>$ & - & - & $>$ & - & - & $>$ & $>$ & - & $>$ & - & $>$ & - & - \\
\hline 116 & - & - & - & - & - & - & - & - & $>$ & - & - & - & - & - & - & - \\
\hline 117 & - & - & - & - & - & - & - & - & - & - & - & - & - & - & - & - \\
\hline 118 & - & - & - & - & - & - & - & - & - & - & - & - & - & - & - & - \\
\hline 119 & - & - & - & - & - & - & - & - & - & - & - & - & - & - & - & - \\
\hline 120 & - & - & - & - & - & - & - & - & - & - & - & - & - & - & - & - \\
\hline 121 & - & - & - & - & - & - & - & - & - & - & - & - & - & - & - & - \\
\hline 122 & - & - & - & - & - & - & - & - & - & - & - & - & - & - & - & - \\
\hline 123 & - & - & - & - & - & - & - & - & - & - & - & - & - & - & - & - \\
\hline 124 & - & - & - & - & - & - & - & - & - & - & - & - & - & - & - & - \\
\hline 125 & - & $>$ & $>$ & - & - & - & - & - & $>$ & $>$ & - & $>$ & - & - & - & - \\
\hline 126 & - & - & $>$ & - & - & - & - & - & $>$ & $>$ & - & $>$ & - & - & - & - \\
\hline 127 & - & $>$ & $>$ & - & - & - & - & - & $>$ & $>$ & - & $>$ & - & - & - & - \\
\hline 128 & - & - & - & - & - & - & - & - & - & - & - & - & - & - & - & - \\
\hline 129 & - & - & - & - & - & - & - & - & - & - & - & - & - & - & - & - \\
\hline 130 & - & $>$ & $>$ & - & - & $>$ & - & - & $>$ & $>$ & - & $>$ & - & $>$ & - & - \\
\hline 131 & - & - & $>$ & - & - & - & - & - & $>$ & $>$ & - & $>$ & - & - & - & - \\
\hline 132 & - & - & - & - & - & - & - & - & - & - & - & - & - & - & - & - \\
\hline
\end{tabular}


Table 3 (Continued from previous page)

\begin{tabular}{|c|c|c|c|c|c|c|c|c|c|c|c|c|c|c|c|c|}
\hline & 17 & 18 & 19 & 20 & 21 & 22 & 23 & 24 & 25 & 26 & 27 & 28 & 29 & 30 & 31 & 32 \\
\hline 133 & - & - & - & - & - & - & - & - & - & - & - & - & - & - & - & - \\
\hline 134 & - & - & - & - & - & - & - & - & - & - & - & - & - & - & - & - \\
\hline 135 & - & - & - & - & - & - & - & - & - & - & - & - & - & - & - & - \\
\hline 136 & - & - & - & - & - & - & - & - & - & - & - & - & - & - & - & - \\
\hline 137 & - & - & - & - & - & - & - & - & - & - & - & - & - & - & - & - \\
\hline 138 & - & - & - & - & - & - & - & - & - & - & - & - & - & - & - & - \\
\hline 139 & $<$ & - & - & - & - & - & - & - & - & - & - & - & - & - & - & - \\
\hline 140 & $<$ & - & - & - & - & - & - & - & - & - & - & - & - & - & - & - \\
\hline 141 & $<$ & - & - & - & - & - & - & - & - & - & - & - & - & - & - & - \\
\hline 142 & - & - & - & - & - & - & - & - & - & - & - & - & - & - & - & - \\
\hline 143 & - & - & - & - & - & - & - & - & - & - & - & - & - & - & - & - \\
\hline 144 & - & - & - & - & - & $<$ & - & - & - & - & - & - & - & - & - & - \\
\hline 145 & $<$ & $<$ & - & - & - & $<$ & - & - & - & - & - & - & - & - & - & - \\
\hline 146 & - & - & - & - & - & - & - & - & - & - & - & - & - & - & - & - \\
\hline 147 & - & - & - & - & - & - & - & - & - & - & - & - & - & - & - & - \\
\hline 148 & - & - & - & - & - & $<$ & - & - & - & - & - & - & - & - & - & - \\
\hline 149 & $<$ & $<$ & - & - & - & $<$ & - & - & - & - & - & - & - & - & - & - \\
\hline 166 & $<$ & - & - & - & - & - & - & - & - & - & - & - & - & $<$ & - & - \\
\hline 167 & - & - & - & - & - & - & - & - & - & - & - & - & - & $<$ & - & - \\
\hline 168 & - & - & - & - & - & - & - & - & - & - & - & - & - & $<$ & - & - \\
\hline 169 & $<$ & $<$ & - & - & - & $<$ & - & - & - & - & - & - & - & - & - & - \\
\hline 170 & - & - & - & - & - & $<$ & - & - & - & - & - & - & - & - & - & - \\
\hline 171 & - & - & - & - & - & - & $<$ & - & - & - & - & - & - & - & $<$ & $<$ \\
\hline 172 & - & - & - & $<$ & - & - & - & - & - & - & - & - & - & - & $<$ & $<$ \\
\hline 173 & - & - & - & - & - & - & - & $>$ & - & - & - & - & - & - & $<$ & $<$ \\
\hline 174 & - & - & - & $<$ & - & - & $<$ & $<$ & - & - & $<$ & - & - & - & $<$ & $<$ \\
\hline 175 & - & - & - & - & - & - & $<$ & - & - & - & - & - & - & - & $<$ & $<$ \\
\hline 176 & - & $>$ & $>$ & - & - & - & - & - & $>$ & $>$ & - & $>$ & - & - & - & - \\
\hline 177 & - & - & - & - & - & - & - & - & - & - & - & - & - & - & - & - \\
\hline 178 & - & $>$ & $>$ & - & - & $>$ & - & $>$ & $>$ & $>$ & - & $>$ & - & $>$ & $>$ & $>$ \\
\hline 179 & - & - & - & - & - & - & - & - & - & - & - & - & - & - & - & - \\
\hline 180 & - & $>$ & $>$ & - & - & $>$ & - & - & - & - & - & - & - & - & - & - \\
\hline 181 & - & - & $>$ & - & - & - & - & - & - & $>$ & - & - & - & - & - & $<$ \\
\hline 182 & - & - & - & $<$ & - & - & - & - & - & - & - & - & - & - & - & $<$ \\
\hline 183 & - & - & $>$ & - & - & - & - & - & - & $>$ & - & - & - & - & - & $<$ \\
\hline 184 & - & - & $>$ & - & - & $<$ & $<$ & - & - & - & - & - & - & - & - & $<$ \\
\hline 185 & $<$ & $<$ & $<$ & $<$ & - & $<$ & $<$ & - & - & $<$ & $<$ & $<$ & - & - & - & $<$ \\
\hline
\end{tabular}


Table 3 (Continued from previous page)

\begin{tabular}{|l|cccccccccccccccc|}
\hline & 17 & 18 & 19 & 20 & 21 & 22 & 23 & 24 & 25 & 26 & 27 & 28 & 29 & 30 & 31 & 32 \\
\hline 186 & - & $>$ & $>$ & - & - & - & - & - & $>$ & $>$ & - & $>$ & - & - & - & - \\
\hline 187 & - & $>$ & $>$ & - & - & $>$ & - & - & - & - & - & - & - & - & - & - \\
\hline 188 & - & $>$ & $>$ & - & - & $>$ & - & $>$ & $>$ & $>$ & - & $>$ & - & $>$ & $>$ & $>$ \\
\hline 189 & - & - & - & - & - & - & - & - & - & - & - & - & - & - & - & - \\
\hline 190 & - & $>$ & $>$ & - & - & $>$ & - & - & - & - & - & - & - & - & - & - \\
\hline 191 & - & - & - & - & - & - & - & - & - & - & - & - & - & - & - & - \\
\hline 192 & $<$ & - & - & - & - & - & - & - & - & - & - & - & - & - & - & - \\
\hline 193 & $<$ & $<$ & - & - & - & $<$ & - & - & - & - & - & - & - & - & - & - \\
\hline 194 & - & - & - & - & - & - & - & - & - & - & - & - & - & - & - & - \\
\hline 195 & - & - & - & - & - & - & - & - & - & - & - & - & - & - & - & - \\
\hline 196 & - & - & - & - & - & - & - & - & - & - & - & - & - & - & - & - \\
\hline 197 & $<$ & $<$ & - & - & - & $<$ & - & - & - & - & - & - & - & - & - & - \\
\hline 198 & - & - & - & - & - & $<$ & - & - & - & - & - & - & - & - & - & - \\
\hline
\end{tabular}

Table 4. Relations between the intuitionistic fuzzy implications $\rightarrow_{33}, \ldots, \rightarrow_{47}$

\begin{tabular}{|c|c|c|c|c|c|c|c|c|c|c|c|c|c|c|c|}
\hline & 33 & 34 & 35 & 36 & 37 & 38 & 39 & 40 & 41 & 42 & 43 & 44 & 45 & 46 & 47 \\
\hline 1 & $<$ & $<$ & - & - & - & - & - & - & - & - & - & - & - & - & - \\
\hline 2 & - & $<$ & - & - & - & - & - & - & - & $<$ & - & - & - & - & $>$ \\
\hline 3 & - & $<$ & - & - & - & - & - & - & - & $<$ & - & - & - & - & $>$ \\
\hline 4 & $<$ & $<$ & - & - & - & - & - & - & - & - & - & - & - & $>$ & - \\
\hline 5 & - & $<$ & - & - & - & - & - & - & - & - & - & - & - & $>$ & $>$ \\
\hline 6 & - & $<$ & - & - & - & - & - & - & - & - & - & - & - & - & - \\
\hline 7 & $<$ & $<$ & - & - & - & - & - & - & - & - & - & - & - & - & - \\
\hline 8 & - & $<$ & - & - & $<$ & - & - & - & - & $<$ & - & - & - & - & - \\
\hline 9 & - & - & - & - & - & - & - & - & - & - & - & - & - & - & - \\
\hline 10 & $<$ & $<$ & - & - & - & - & $<$ & - & - & - & - & - & - & - & - \\
\hline 11 & - & $<$ & - & - & - & - & - & - & - & $<$ & - & - & - & - & $>$ \\
\hline 12 & $<$ & $<$ & - & - & - & - & - & - & - & - & - & - & - & $>$ & - \\
\hline 13 & - & $<$ & - & - & - & - & - & - & - & - & - & - & - & $>$ & - \\
\hline 14 & - & $<$ & - & - & - & - & - & - & - & $<$ & - & - & - & - & $>$ \\
\hline 15 & - & $<$ & - & - & $<$ & - & - & $<$ & - & $<$ & - & - & - & - & - \\
\hline 16 & $<$ & $<$ & - & - & - & - & - & - & $<$ & $<$ & $<$ & $<$ & - & - & - \\
\hline 17 & - & - & - & - & - & - & - & - & - & - & - & - & - & $>$ & - \\
\hline 18 & $<$ & $<$ & - & - & - & - & - & - & - & - & - & - & - & $>$ & - \\
\hline 19 & $<$ & $<$ & - & - & - & - & - & - & $<$ & $<$ & $<$ & $<$ & - & - & - \\
\hline 20 & - & - & - & - & - & - & - & - & - & $<$ & - & - & - & - & $>$ \\
\hline 21 & - & - & - & - & - & - & - & - & - & - & - & - & - & - & - \\
\hline
\end{tabular}


Table 4 (Continued from previous page)

\begin{tabular}{|c|c|c|c|c|c|c|c|c|c|c|c|c|c|c|c|}
\hline & 33 & 34 & 35 & 36 & 37 & 38 & 39 & 40 & 41 & 42 & 43 & 44 & 45 & 46 & 47 \\
\hline 22 & $<$ & $<$ & - & - & - & - & - & - & - & - & - & - & - & $>$ & - \\
\hline 23 & - & - & - & - & - & - & - & - & - & $<$ & - & - & - & - & $>$ \\
\hline 24 & - & $<$ & - & - & $<$ & - & - & $<$ & - & $<$ & - & - & - & - & - \\
\hline 25 & $<$ & $<$ & - & - & - & - & - & - & - & - & $<$ & $<$ & $<$ & - & - \\
\hline 26 & $<$ & $<$ & - & - & - & - & - & - & $<$ & $<$ & $<$ & $<$ & - & - & - \\
\hline 27 & - & - & - & - & - & - & - & - & - & $<$ & - & - & - & - & $>$ \\
\hline 28 & $<$ & $<$ & - & - & - & - & - & - & - & - & $<$ & $<$ & - & - & - \\
\hline 29 & - & - & - & - & - & - & - & - & - & - & - & - & $<$ & - & - \\
\hline 30 & $<$ & $<$ & - & $>$ & - & - & - & - & - & - & - & - & - & - & - \\
\hline 31 & - & $<$ & - & - & - & - & - & $>$ & - & $<$ & - & - & - & - & $>$ \\
\hline 32 & - & $<$ & - & - & $>$ & - & - & $>$ & $>$ & $<$ & - & - & - & - & $>$ \\
\hline 33 & $=$ & $<$ & - & $>$ & - & - & $>$ & - & $>$ & - & $>$ & $>$ & - & $>$ & - \\
\hline 34 & $>$ & $=$ & $>$ & $>$ & $>$ & $>$ & $>$ & $>$ & $>$ & - & $>$ & $>$ & - & $>$ & $>$ \\
\hline 35 & - & $<$ & $=$ & - & - & - & - & - & - & - & - & - & - & - & - \\
\hline 36 & $<$ & $<$ & - & $=$ & - & - & - & - & - & - & - & - & - & - & - \\
\hline 37 & - & $<$ & - & - & $=$ & - & - & $>$ & - & $<$ & - & - & - & - & - \\
\hline 38 & - & $<$ & - & - & - & $=$ & - & - & - & - & - & - & - & - & - \\
\hline 39 & $<$ & $<$ & - & - & - & - & $=$ & - & - & - & - & - & - & - & - \\
\hline 40 & - & $<$ & - & - & $<$ & - & - & $=$ & - & $<$ & - & - & - & - & - \\
\hline 41 & $<$ & $<$ & - & - & - & - & - & - & $=$ & $<$ & $<$ & $<$ & - & - & - \\
\hline 42 & - & - & - & - & $>$ & - & - & $>$ & $>$ & $=$ & - & - & - & - & $>$ \\
\hline 43 & $<$ & $<$ & - & - & - & - & - & - & $>$ & - & $=$ & $"="$ & - & - & - \\
\hline 44 & $<$ & $<$ & - & - & - & - & - & - & $>$ & - & $"="$ & $=$ & - & - & - \\
\hline 45 & - & - & - & - & - & - & - & - & - & - & - & - & $=$ & - & - \\
\hline 46 & $<$ & $<$ & - & - & - & - & - & - & - & - & - & - & - & $=$ & - \\
\hline 47 & - & $<$ & - & - & - & - & - & - & - & $<$ & - & - & - & - & $=$ \\
\hline 48 & - & $<$ & - & - & - & - & - & - & - & $<$ & - & - & - & - & $>$ \\
\hline 49 & - & $<$ & - & - & - & - & - & - & - & - & - & - & - & $>$ & $>$ \\
\hline 50 & - & $<$ & - & - & - & - & - & - & - & - & - & - & - & $>$ & - \\
\hline 51 & $<$ & $<$ & - & - & - & - & - & - & - & - & - & - & - & $<$ & - \\
\hline 52 & - & $<$ & - & - & $<$ & - & - & - & - & $<$ & - & - & - & - & - \\
\hline 53 & - & $<$ & - & - & - & - & - & - & - & - & - & - & - & - & - \\
\hline 54 & $<$ & $<$ & - & - & - & - & - & - & - & - & - & - & - & - & - \\
\hline 55 & - & $<$ & - & - & $<$ & - & - & $<$ & - & $<$ & - & - & - & - & $<$ \\
\hline 56 & $<$ & $<$ & - & - & - & - & - & - & $<$ & $<$ & $<$ & $<$ & - & - & - \\
\hline 57 & - & - & - & - & - & - & - & - & - & $<$ & - & - & - & - & $>$ \\
\hline 58 & $<$ & $<$ & - & - & - & - & - & - & - & - & $<$ & $<$ & $<$ & - & - \\
\hline
\end{tabular}


Table 4 (Continued from previous page)

\begin{tabular}{|c|c|c|c|c|c|c|c|c|c|c|c|c|c|c|c|}
\hline & 33 & 34 & 35 & 36 & 37 & 38 & 39 & 40 & 41 & 42 & 43 & 44 & 45 & 46 & 47 \\
\hline 59 & $<$ & $<$ & - & - & - & - & - & - & - & - & $<$ & $<$ & - & - & - \\
\hline 60 & - & - & - & - & - & - & - & - & - & - & - & - & $<$ & - & - \\
\hline 61 & $<$ & $<$ & - & - & - & - & - & - & - & - & - & - & - & - & - \\
\hline 62 & - & $<$ & - & - & - & - & - & - & - & - & - & - & - & - & - \\
\hline 63 & - & $<$ & - & - & - & - & - & - & - & - & - & - & - & - & - \\
\hline 64 & - & $<$ & - & - & - & - & - & - & - & - & - & - & - & - & - \\
\hline 65 & - & $<$ & - & - & $<$ & - & - & - & - & $<$ & - & - & - & - & - \\
\hline 66 & - & - & - & - & - & - & - & - & - & - & - & - & - & - & - \\
\hline 67 & $<$ & $<$ & - & - & - & - & - & - & - & - & - & - & - & - & - \\
\hline 68 & - & $<$ & - & - & - & - & - & - & - & - & - & - & - & - & - \\
\hline 69 & - & $<$ & - & - & - & - & - & - & - & - & - & - & - & - & - \\
\hline 70 & $<$ & $<$ & - & - & - & - & $<$ & - & - & - & - & - & - & - & - \\
\hline 71 & - & - & - & - & - & - & - & - & - & - & - & - & - & $>$ & - \\
\hline 72 & $<$ & $<$ & - & - & - & - & - & - & - & - & - & - & - & $>$ & - \\
\hline 73 & $<$ & $<$ & - & - & - & - & $<$ & - & - & - & - & - & - & - & - \\
\hline 74 & - & - & - & - & - & - & - & - & - & - & - & - & - & - & - \\
\hline 75 & - & - & - & - & - & - & - & - & - & - & - & - & - & - & - \\
\hline 76 & $<$ & $<$ & - & - & - & - & - & - & - & - & - & - & - & $>$ & - \\
\hline 77 & - & - & - & - & - & - & - & - & - & - & - & - & - & - & - \\
\hline 78 & $<$ & $<$ & - & - & - & - & $<$ & - & - & - & - & - & - & - & - \\
\hline 79 & - & - & - & - & - & - & - & - & - & - & - & - & - & - & - \\
\hline 80 & $<$ & $<$ & - & - & - & - & - & - & - & - & - & - & - & - & - \\
\hline 81 & - & - & - & - & - & - & - & - & - & - & - & - & - & - & - \\
\hline 82 & $<$ & $<$ & - & $>$ & - & - & - & - & - & - & - & - & - & - & - \\
\hline 83 & - & $<$ & - & - & - & - & - & $>$ & - & - & - & - & - & - & - \\
\hline 84 & - & $<$ & - & - & $>$ & - & - & $>$ & - & - & - & - & - & - & - \\
\hline 85 & - & $<$ & - & - & - & - & - & - & - & - & - & - & - & - & - \\
\hline 86 & $<$ & $<$ & - & - & - & - & - & - & $>$ & - & - & - & - & - & - \\
\hline 87 & $<$ & $<$ & - & - & - & - & $<$ & - & - & - & - & - & - & - & - \\
\hline 88 & - & - & - & - & $>$ & - & - & $>$ & - & - & - & - & - & - & - \\
\hline 89 & $<$ & $<$ & - & - & - & - & - & - & - & - & - & - & - & - & - \\
\hline 90 & - & - & - & - & - & - & - & - & - & - & - & - & - & - & - \\
\hline 91 & $<$ & $<$ & - & - & - & - & - & - & - & - & - & - & - & - & - \\
\hline 92 & - & $<$ & - & - & - & - & - & - & - & - & - & - & - & - & - \\
\hline 93 & - & $<$ & - & - & - & - & - & - & - & - & - & - & - & - & - \\
\hline 94 & - & $<$ & - & - & - & - & - & - & - & - & - & - & - & - & - \\
\hline 95 & $<$ & $<$ & - & - & - & - & - & - & - & - & - & - & - & - & - \\
\hline
\end{tabular}


Table 4 (Continued from previous page)

\begin{tabular}{|c|c|c|c|c|c|c|c|c|c|c|c|c|c|c|c|}
\hline & 33 & 34 & 35 & 36 & 37 & 38 & 39 & 40 & 41 & 42 & 43 & 44 & 45 & 46 & 47 \\
\hline 96 & $<$ & $<$ & - & - & - & - & $<$ & - & - & - & - & - & - & - & - \\
\hline 97 & - & - & - & - & - & - & - & - & - & - & - & - & - & - & - \\
\hline 98 & $<$ & $<$ & - & - & - & - & - & - & - & - & - & - & - & - & - \\
\hline 99 & - & - & - & - & - & - & - & - & - & - & - & - & - & - & - \\
\hline 100 & - & - & - & - & - & - & - & - & - & - & - & - & - & - & - \\
\hline 101 & - & - & - & - & - & - & - & - & - & - & - & - & - & - & - \\
\hline 102 & - & - & - & - & - & - & - & - & - & - & - & - & - & - & - \\
\hline 103 & - & - & - & - & - & - & - & - & - & - & - & - & - & - & - \\
\hline 104 & - & - & - & - & - & - & - & - & - & - & - & - & - & - & - \\
\hline 105 & - & - & - & - & - & - & - & - & - & - & - & - & - & - & - \\
\hline 106 & - & - & - & - & - & - & - & - & - & - & - & - & - & - & - \\
\hline 107 & - & - & - & - & - & - & - & - & - & - & - & - & - & - & - \\
\hline 108 & - & - & - & - & - & - & - & - & - & - & - & - & - & - & - \\
\hline 109 & - & - & - & - & - & - & - & - & - & - & - & - & - & - & - \\
\hline 110 & - & - & - & - & - & - & - & - & - & - & - & - & - & $>$ & - \\
\hline 111 & - & - & - & - & - & - & - & - & - & - & - & - & - & - & - \\
\hline 112 & - & - & - & - & - & - & - & - & - & - & - & - & - & $>$ & - \\
\hline 113 & - & - & - & - & - & - & - & - & - & - & - & - & - & - & - \\
\hline 114 & - & - & - & - & - & - & - & - & - & - & - & - & - & - & - \\
\hline 115 & $>$ & - & - & $>$ & - & - & $>$ & - & $>$ & - & $>$ & $>$ & - & $>$ & - \\
\hline 116 & - & - & $>$ & - & - & - & - & - & - & - & - & - & - & - & - \\
\hline 117 & - & - & - & - & - & - & - & - & - & - & - & - & - & - & - \\
\hline 118 & - & - & - & - & - & - & - & - & - & - & - & - & - & - & - \\
\hline 119 & - & - & - & - & - & - & - & - & - & - & - & - & - & - & - \\
\hline 120 & - & - & - & - & - & - & - & - & - & - & - & - & - & $>$ & - \\
\hline 121 & - & - & - & - & - & - & - & - & - & - & - & - & - & - & - \\
\hline 122 & - & - & - & - & - & - & - & - & - & - & - & - & - & $>$ & - \\
\hline 123 & - & - & - & - & - & - & - & - & - & - & - & - & - & - & - \\
\hline 124 & - & - & - & - & - & - & - & - & - & - & - & - & - & - & - \\
\hline 125 & - & - & - & - & - & - & - & - & - & - & - & - & - & $>$ & - \\
\hline 126 & - & - & - & - & - & - & - & - & - & - & - & - & - & - & - \\
\hline 127 & - & - & - & - & - & - & - & - & - & - & - & - & - & $>$ & - \\
\hline 128 & - & - & - & - & - & - & - & - & - & - & - & - & - & - & - \\
\hline 129 & - & - & - & - & - & - & - & - & - & - & - & - & - & - & - \\
\hline 130 & $>$ & - & - & $>$ & - & - & $>$ & - & $>$ & - & $>$ & $>$ & - & $>$ & - \\
\hline 131 & - & - & - & - & - & - & - & - & $>$ & - & $>$ & $>$ & - & - & - \\
\hline 132 & - & - & $>$ & - & - & - & - & - & - & - & - & - & - & - & - \\
\hline
\end{tabular}


Table 4 (Continued from previous page)

\begin{tabular}{|c|c|c|c|c|c|c|c|c|c|c|c|c|c|c|c|}
\hline & 33 & 34 & 35 & 36 & 37 & 38 & 39 & 40 & 41 & 42 & 43 & 44 & 45 & 46 & 47 \\
\hline 133 & - & - & - & - & - & - & - & - & - & - & - & - & - & - & - \\
\hline 134 & - & - & - & - & - & - & - & - & - & - & - & - & - & - & - \\
\hline 135 & - & - & - & - & - & - & - & - & - & - & - & - & - & $>$ & - \\
\hline 136 & - & - & - & - & - & - & - & - & - & - & - & - & - & - & - \\
\hline 137 & - & - & - & - & - & - & - & - & - & - & - & - & - & $>$ & - \\
\hline 138 & - & - & - & - & - & - & - & - & - & - & - & - & - & - & - \\
\hline 139 & $<$ & $<$ & - & - & - & - & - & - & - & - & - & - & - & - & - \\
\hline 140 & $<$ & $<$ & - & - & - & - & - & - & - & - & - & - & - & - & - \\
\hline 141 & $<$ & $<$ & - & - & - & - & - & - & - & - & - & - & - & - & - \\
\hline 142 & $<$ & $<$ & - & - & - & - & - & - & - & - & - & - & - & - & - \\
\hline 143 & $<$ & $<$ & - & - & - & - & - & - & - & - & - & - & - & - & - \\
\hline 144 & $<$ & $<$ & - & - & - & - & - & - & - & - & - & - & - & - & - \\
\hline 145 & $<$ & $<$ & - & - & - & - & - & - & - & - & - & - & - & - & - \\
\hline 146 & $<$ & $<$ & - & - & - & - & - & - & - & - & - & - & - & - & - \\
\hline 147 & $<$ & $<$ & - & - & - & - & - & - & - & - & - & - & - & - & - \\
\hline 148 & $<$ & $<$ & - & - & - & - & - & - & - & - & - & - & - & - & - \\
\hline 149 & $<$ & $<$ & - & - & - & - & - & - & - & - & - & - & - & - & - \\
\hline 166 & $<$ & $<$ & - & - & - & - & - & - & - & - & - & - & - & - & - \\
\hline 167 & $<$ & $<$ & - & - & - & - & - & - & - & - & - & - & - & - & - \\
\hline 168 & $<$ & $<$ & - & $>$ & - & - & - & - & - & - & - & - & - & - & - \\
\hline 169 & $<$ & $<$ & - & - & - & - & - & - & - & - & - & - & - & $<$ & - \\
\hline 170 & $<$ & $<$ & - & - & - & - & - & - & - & - & - & - & - & - & - \\
\hline 171 & - & $<$ & - & - & $<$ & - & - & $<$ & - & $<$ & - & - & - & - & - \\
\hline 172 & - & $<$ & - & - & $<$ & - & - & $<$ & - & $<$ & - & - & - & - & - \\
\hline 173 & - & $<$ & - & - & $<$ & - & - & $"="$ & - & $<$ & - & - & - & - & - \\
\hline 174 & - & $<$ & - & - & $<$ & - & - & $<$ & - & $<$ & - & - & - & - & $<$ \\
\hline 175 & - & $<$ & - & - & $<$ & - & - & $<$ & - & $<$ & - & - & - & - & - \\
\hline 176 & - & $<$ & - & - & - & - & - & - & - & - & - & - & - & $>$ & $>$ \\
\hline 177 & - & $<$ & - & - & - & - & - & - & - & - & - & - & - & $>$ & $>$ \\
\hline 178 & $>$ & $<$ & - & $>$ & $>$ & - & $>$ & $>$ & $>$ & - & $>$ & $>$ & - & $>$ & $>$ \\
\hline 179 & - & $<$ & - & - & - & - & - & - & - & - & - & - & - & $>$ & $>$ \\
\hline 180 & - & $<$ & - & - & - & - & - & - & - & - & - & - & - & $>$ & $>$ \\
\hline 181 & $<$ & $<$ & - & - & - & - & - & - & $<$ & $<$ & $<$ & $<$ & - & - & - \\
\hline 182 & $<$ & $<$ & - & - & - & - & - & - & $<$ & $<$ & $<$ & $<$ & - & - & - \\
\hline 183 & $<$ & $<$ & - & - & - & - & - & - & $"="$ & $<$ & $<$ & $<$ & - & - & - \\
\hline 184 & $<$ & $<$ & - & - & - & - & - & - & $<$ & $<$ & $<$ & $<$ & - & - & - \\
\hline 185 & $<$ & $<$ & - & - & - & - & - & - & $<$ & $<$ & $<$ & $<$ & - & - & - \\
\hline
\end{tabular}


Table 4 (Continued from previous page)

\begin{tabular}{|l|ccccccccccccccc|}
\hline & 33 & 34 & 35 & 36 & 37 & 38 & 39 & 40 & 41 & 42 & 43 & 44 & 45 & 46 & 47 \\
\hline 186 & - & $<$ & - & - & - & - & - & - & - & - & - & - & - & $>$ & $>$ \\
\hline 187 & - & $<$ & - & - & - & - & - & - & - & - & - & - & - & $>$ & $>$ \\
\hline 188 & $>$ & $<$ & - & $>$ & $>$ & - & $>$ & $>$ & $>$ & - & $>$ & $>$ & - & $>$ & $>$ \\
\hline 189 & - & $<$ & - & - & - & - & - & - & - & - & - & - & - & $>$ & $>$ \\
\hline 190 & - & $<$ & - & - & - & - & - & - & - & - & - & - & - & $>$ & $>$ \\
\hline 191 & - & $<$ & - & - & - & - & - & - & - & - & - & - & - & - & $>$ \\
\hline 192 & $<$ & $<$ & - & - & - & - & - & - & - & - & - & - & - & - & - \\
\hline 193 & $<$ & $<$ & - & - & - & - & - & - & - & - & - & - & - & - & - \\
\hline 194 & $<$ & $<$ & - & $>$ & - & - & - & - & - & - & - & - & - & - & - \\
\hline 195 & $<$ & $<$ & - & - & - & - & - & - & - & - & - & - & - & - & - \\
\hline 196 & $<$ & $<$ & - & $>$ & - & - & - & - & - & - & - & - & - & - & - \\
\hline 197 & $<$ & $<$ & - & - & - & - & - & - & - & - & - & - & - & - & - \\
\hline 198 & $<$ & $<$ & - & - & - & - & - & - & - & - & - & - & - & - & - \\
\hline
\end{tabular}

Table 5. Relations between the intuitionistic fuzzy implications $\rightarrow_{48}, \ldots, \rightarrow_{62}$

\begin{tabular}{|c|ccccccccccccccc|}
\hline & 48 & 49 & 50 & 51 & 52 & 53 & 54 & 55 & 56 & 57 & 58 & 59 & 60 & 61 & 62 \\
\hline 1 & - & - & - & - & - & - & - & - & - & - & - & - & - & - & - \\
\hline 2 & - & - & - & - & - & - & - & $>$ & - & - & - & - & - & - & - \\
\hline 3 & $>$ & - & - & - & $>$ & - & - & $>$ & $>$ & - & - & - & - & - & - \\
\hline 4 & - & - & - & $>$ & - & - & $>$ & - & $>$ & - & $>$ & $>$ & - & $>$ & - \\
\hline 5 & $>$ & $>$ & $>$ & $>$ & $>$ & $>$ & $>$ & $>$ & $>$ & - & $>$ & $>$ & - & $>$ & - \\
\hline 6 & - & - & - & - & - & - & - & - & - & - & - & - & - & - & - \\
\hline 7 & - & - & - & - & - & - & - & - & - & - & - & - & - & $<$ & - \\
\hline 8 & - & - & - & - & $>$ & - & - & $>$ & - & - & - & - & - & - & - \\
\hline 9 & - & - & - & - & - & - & - & - & - & - & - & - & - & - & - \\
\hline 10 & - & - & - & - & - & - & - & - & - & - & - & - & - & - & - \\
\hline 11 & $>$ & - & - & - & $>$ & - & - & $>$ & $>$ & - & - & - & - & - & - \\
\hline 12 & - & $<$ & $<$ & $>$ & - & - & $>$ & - & $>$ & - & $>$ & $>$ & - & - & - \\
\hline 13 & - & - & $>$ & $>$ & - & $>$ & $>$ & - & $>$ & - & $>$ & $>$ & - & $>$ & - \\
\hline 14 & $>$ & - & - & - & $>$ & - & - & $>$ & $>$ & - & - & - & - & - & - \\
\hline 15 & - & - & - & - & - & - & - & $>$ & - & - & - & - & - & - & - \\
\hline 16 & - & - & - & - & - & - & - & - & $>$ & - & - & - & - & - & - \\
\hline 17 & - & - & - & $>$ & - & - & $>$ & - & $>$ & - & $>$ & $>$ & - & $>$ & - \\
\hline 18 & - & - & - & $>$ & - & - & $>$ & - & $>$ & - & $>$ & $>$ & - & - & - \\
\hline 19 & - & - & - & - & - & - & - & - & $>$ & - & - & - & - & - & - \\
\hline 20 & $>$ & - & - & - & $>$ & - & - & $>$ & $>$ & $>$ & - & - & - & - & - \\
\hline 21 & - & - & - & - & - & - & - & - & - & - & $>$ & - & - & - & - \\
\hline & & & & & $($ Continued on next page $)$ & & & & \\
\hline
\end{tabular}


Table 5 (Continued from previous page)

\begin{tabular}{|c|c|c|c|c|c|c|c|c|c|c|c|c|c|c|c|}
\hline & 48 & 49 & 50 & 51 & 52 & 53 & 54 & 55 & 56 & 57 & 58 & 59 & 60 & 61 & 62 \\
\hline 22 & - & - & - & $>$ & - & - & $>$ & - & $>$ & - & $>$ & $>$ & - & - & - \\
\hline 23 & $>$ & - & - & - & $>$ & - & - & $>$ & $>$ & $>$ & - & - & - & - & - \\
\hline 24 & - & - & - & - & - & - & - & $>$ & - & - & - & - & - & - & - \\
\hline 25 & - & - & - & - & - & - & - & - & - & - & $>$ & - & - & - & - \\
\hline 26 & - & - & - & - & - & - & - & - & $>$ & - & - & - & - & - & - \\
\hline 27 & $>$ & - & - & - & $>$ & - & - & $>$ & $>$ & $>$ & - & - & - & - & - \\
\hline 28 & - & - & - & - & - & - & - & - & $>$ & - & $>$ & $>$ & - & - & - \\
\hline 29 & - & - & - & - & - & - & - & - & - & - & $>$ & - & $>$ & - & - \\
\hline 30 & - & - & - & - & - & - & - & - & - & - & - & - & - & - & - \\
\hline 31 & - & - & - & - & - & - & - & $>$ & - & - & - & - & - & - & - \\
\hline 32 & $>$ & - & - & - & $>$ & - & - & $>$ & $>$ & - & - & - & - & - & - \\
\hline 33 & - & - & - & $>$ & - & - & $>$ & - & $>$ & - & $>$ & $>$ & - & $>$ & - \\
\hline 34 & $>$ & $>$ & $>$ & $>$ & $>$ & $>$ & $>$ & $>$ & $>$ & - & $>$ & $>$ & - & $>$ & $>$ \\
\hline 35 & - & - & - & - & - & - & - & - & - & - & - & - & - & - & - \\
\hline 36 & - & - & - & - & - & - & - & - & - & - & - & - & - & - & - \\
\hline 37 & - & - & - & - & $>$ & - & - & $>$ & - & - & - & - & - & - & - \\
\hline 38 & - & - & - & - & - & - & - & - & - & - & - & - & - & - & - \\
\hline 39 & - & - & - & - & - & - & - & - & - & - & - & - & - & - & - \\
\hline 40 & - & - & - & - & - & - & - & $>$ & - & - & - & - & - & - & - \\
\hline 41 & - & - & - & - & - & - & - & - & $>$ & - & - & - & - & - & - \\
\hline 42 & $>$ & - & - & - & $>$ & - & - & $>$ & $>$ & $>$ & - & - & - & - & - \\
\hline 43 & - & - & - & - & - & - & - & - & $>$ & - & $>$ & $>$ & - & - & - \\
\hline 44 & - & - & - & - & - & - & - & - & $>$ & - & $>$ & $>$ & - & - & - \\
\hline 45 & - & - & - & - & - & - & - & - & - & - & $>$ & - & $>$ & - & - \\
\hline 46 & - & $<$ & $<$ & $>$ & - & - & - & - & - & - & - & - & - & - & - \\
\hline 47 & $<$ & $<$ & - & - & - & - & - & $>$ & - & $<$ & - & - & - & - & - \\
\hline 48 & $=$ & $<$ & - & - & $>$ & - & - & $>$ & $>$ & $<$ & - & - & - & - & - \\
\hline 49 & $>$ & $=$ & $>$ & $>$ & $>$ & $>$ & $>$ & $>$ & $>$ & - & $>$ & $>$ & - & - & - \\
\hline 50 & - & $<$ & $=$ & $>$ & - & $>$ & $>$ & - & $>$ & - & $>$ & $>$ & - & - & - \\
\hline 51 & - & $<$ & $<$ & $=$ & - & - & - & - & - & - & - & - & - & - & - \\
\hline 52 & $<$ & $<$ & - & - & $=$ & - & - & $>$ & - & $<$ & - & - & - & - & - \\
\hline 53 & - & $<$ & $<$ & - & - & $=$ & - & - & - & - & - & - & - & - & - \\
\hline 54 & - & $<$ & $<$ & - & - & - & $=$ & - & - & - & - & - & - & - & - \\
\hline 55 & $<$ & $<$ & - & - & $<$ & - & - & $=$ & - & $<$ & - & - & - & - & $<$ \\
\hline 56 & $<$ & $<$ & $<$ & - & - & - & - & - & $=$ & $<$ & - & $<$ & - & - & - \\
\hline 57 & $>$ & - & - & - & $>$ & - & - & $>$ & $>$ & $=$ & - & - & - & - & - \\
\hline 58 & - & $<$ & $<$ & - & - & - & - & - & - & - & $=$ & $<$ & $<$ & - & - \\
\hline
\end{tabular}


Table 5 (Continued from previous page)

\begin{tabular}{|c|c|c|c|c|c|c|c|c|c|c|c|c|c|c|c|}
\hline & 48 & 49 & 50 & 51 & 52 & 53 & 54 & 55 & 56 & 57 & 58 & 59 & 60 & 61 & 62 \\
\hline 59 & - & $<$ & $<$ & - & - & - & - & - & $>$ & - & $>$ & $=$ & - & - & - \\
\hline 60 & - & - & - & - & - & - & - & - & - & - & $>$ & - & $=$ & - & - \\
\hline 61 & - & - & - & - & - & - & - & - & - & - & - & - & - & $=$ & - \\
\hline 62 & - & - & - & - & - & - & - & $>$ & - & - & - & - & - & - & $=$ \\
\hline 63 & - & - & - & - & $>$ & - & - & $>$ & - & - & - & - & - & - & $>$ \\
\hline 64 & - & - & - & - & - & - & - & - & - & - & - & - & - & - & - \\
\hline 65 & - & - & - & - & $>$ & - & - & $>$ & - & - & - & - & - & - & - \\
\hline 66 & - & - & - & - & - & - & - & - & - & - & - & - & - & - & - \\
\hline 67 & - & - & - & - & - & - & - & - & $>$ & - & - & - & - & - & - \\
\hline 68 & - & - & - & - & $>$ & - & - & $>$ & - & - & - & - & - & - & $>$ \\
\hline 69 & - & - & - & - & $>$ & - & - & $>$ & - & - & - & - & - & - & - \\
\hline 70 & - & - & - & - & - & - & - & - & - & - & - & - & - & - & - \\
\hline 71 & - & - & - & $>$ & - & - & $>$ & - & $>$ & - & $>$ & $>$ & - & $>$ & - \\
\hline 72 & - & - & - & $>$ & - & - & $>$ & - & $>$ & - & $>$ & $>$ & - & - & - \\
\hline 73 & - & - & - & - & - & - & - & - & - & - & - & - & - & - & - \\
\hline 74 & - & - & - & - & $>$ & - & - & $>$ & - & - & - & - & - & - & - \\
\hline 75 & - & - & - & - & - & - & - & - & $>$ & - & $>$ & $>$ & - & - & - \\
\hline 76 & - & - & - & $>$ & - & - & $>$ & - & $>$ & - & $>$ & $>$ & - & - & - \\
\hline 77 & - & - & - & - & $>$ & - & - & $>$ & - & - & - & - & - & - & - \\
\hline 78 & - & - & - & - & - & - & - & - & - & - & - & - & - & - & - \\
\hline 79 & - & - & - & - & $>$ & - & - & $>$ & - & - & - & - & - & - & - \\
\hline 80 & - & - & - & - & - & - & - & - & - & - & $>$ & - & - & - & - \\
\hline 81 & - & - & - & - & - & - & - & - & - & - & $>$ & - & - & - & - \\
\hline 82 & - & - & - & - & - & - & - & - & - & - & - & - & - & $>$ & - \\
\hline 83 & - & - & - & - & - & - & - & $>$ & - & - & - & - & - & - & $>$ \\
\hline 84 & - & - & - & - & $>$ & - & - & $>$ & - & - & - & - & - & - & $>$ \\
\hline 85 & - & - & - & - & - & - & - & - & - & - & - & - & - & - & - \\
\hline 86 & - & - & - & - & - & - & - & - & $>$ & - & - & - & - & - & - \\
\hline 87 & - & - & - & - & - & - & - & - & - & - & - & - & - & - & - \\
\hline 88 & - & - & - & - & $>$ & - & - & $>$ & - & - & - & - & - & - & $>$ \\
\hline 89 & - & - & - & - & - & - & - & - & - & - & $>$ & - & - & - & - \\
\hline 90 & - & - & - & - & - & - & - & - & - & - & $>$ & - & - & - & - \\
\hline 91 & - & $<$ & $<$ & $>$ & - & - & - & - & - & - & - & - & - & - & - \\
\hline 92 & - & $<$ & - & - & - & - & - & $>$ & - & - & - & - & - & - & $<$ \\
\hline 93 & - & $<$ & - & - & $>$ & - & - & $>$ & - & - & - & - & - & - & - \\
\hline 94 & - & $<$ & $<$ & - & - & - & - & - & - & - & - & - & - & - & - \\
\hline 95 & - & $<$ & $<$ & - & - & - & - & - & $>$ & - & - & - & - & - & - \\
\hline
\end{tabular}


Table 5 (Continued from previous page)

\begin{tabular}{|c|c|c|c|c|c|c|c|c|c|c|c|c|c|c|c|}
\hline & 48 & 49 & 50 & 51 & 52 & 53 & 54 & 55 & 56 & 57 & 58 & 59 & 60 & 61 & 62 \\
\hline 96 & - & $<$ & $<$ & - & - & - & $<$ & - & - & - & - & - & - & - & - \\
\hline 97 & - & - & - & - & $>$ & - & - & $>$ & - & - & - & - & - & - & - \\
\hline 98 & - & $<$ & $<$ & - & - & - & - & - & - & - & $>$ & - & - & - & - \\
\hline 99 & - & - & - & - & - & - & - & - & - & - & $>$ & - & - & - & - \\
\hline 100 & - & - & - & - & - & - & - & - & - & - & - & - & - & - & - \\
\hline 101 & - & - & - & - & - & - & - & - & - & - & - & - & - & - & - \\
\hline 102 & - & - & - & - & - & - & - & - & - & - & - & - & - & - & - \\
\hline 103 & - & - & - & - & - & - & - & - & - & - & - & - & - & - & - \\
\hline 104 & - & - & - & - & - & - & - & - & - & - & - & - & - & - & - \\
\hline 105 & - & - & - & - & - & - & - & - & - & - & - & - & - & - & - \\
\hline 106 & - & - & - & - & - & - & - & - & - & - & - & - & - & - & - \\
\hline 107 & - & - & - & - & - & - & - & - & - & - & - & - & - & - & - \\
\hline 108 & - & - & - & - & - & - & - & - & - & - & - & - & - & - & - \\
\hline 109 & - & - & - & - & - & - & - & - & - & - & - & - & - & - & - \\
\hline 110 & - & - & - & $>$ & - & - & $>$ & - & $>$ & - & $>$ & $>$ & - & $>$ & - \\
\hline 111 & - & - & - & - & - & - & - & - & - & - & $>$ & - & - & - & - \\
\hline 112 & - & - & $>$ & $>$ & - & $>$ & $>$ & - & $>$ & - & $>$ & $>$ & - & $>$ & - \\
\hline 113 & - & - & - & - & - & - & - & - & - & - & - & - & - & - & - \\
\hline 114 & - & - & - & - & - & - & - & - & - & - & - & - & - & - & - \\
\hline 115 & - & - & - & $>$ & - & - & $>$ & - & $>$ & - & $>$ & $>$ & - & $>$ & - \\
\hline 116 & - & - & - & - & - & - & - & - & - & - & $>$ & - & - & - & - \\
\hline 117 & - & - & - & - & - & - & - & - & - & - & - & - & - & - & - \\
\hline 118 & - & - & - & - & - & - & - & - & - & - & - & - & - & - & - \\
\hline 119 & - & - & - & - & - & - & - & - & - & - & - & - & - & - & - \\
\hline 120 & - & - & - & $>$ & - & - & $>$ & - & $>$ & - & $>$ & $>$ & - & - & - \\
\hline 121 & - & - & - & - & - & - & - & - & - & - & $>$ & - & - & - & - \\
\hline 122 & - & - & $>$ & $>$ & - & $>$ & $>$ & - & $>$ & - & $>$ & $>$ & - & - & - \\
\hline 123 & - & - & - & - & - & - & - & - & - & - & - & - & - & - & - \\
\hline 124 & - & - & - & - & - & - & - & - & - & - & - & - & - & - & - \\
\hline 125 & - & - & - & $>$ & - & - & $>$ & - & $>$ & - & $>$ & $>$ & - & $>$ & - \\
\hline 126 & - & - & - & - & - & - & - & - & $>$ & - & $>$ & $>$ & - & - & - \\
\hline 127 & - & - & $>$ & $>$ & - & $>$ & $>$ & - & $>$ & - & $>$ & $>$ & - & $>$ & - \\
\hline 128 & - & - & - & - & - & - & - & - & - & - & - & - & - & - & - \\
\hline 129 & - & - & - & - & - & - & - & - & - & - & - & - & - & - & - \\
\hline 130 & - & - & - & $>$ & - & - & $>$ & - & $>$ & - & $>$ & $>$ & - & $>$ & - \\
\hline 131 & - & - & - & - & - & - & - & - & $>$ & - & $>$ & $>$ & - & - & - \\
\hline 132 & - & - & - & - & - & - & - & - & - & - & - & - & - & - & - \\
\hline
\end{tabular}


Table 5 (Continued from previous page)

\begin{tabular}{|c|c|c|c|c|c|c|c|c|c|c|c|c|c|c|c|}
\hline & 48 & 49 & 50 & 51 & 52 & 53 & 54 & 55 & 56 & 57 & 58 & 59 & 60 & 61 & 62 \\
\hline 133 & - & - & - & - & - & - & - & - & - & - & - & - & - & - & - \\
\hline 134 & - & - & - & - & - & - & - & - & - & - & - & - & - & - & - \\
\hline 135 & - & - & - & $>$ & - & - & $>$ & - & $>$ & - & $>$ & $>$ & - & - & - \\
\hline 136 & - & - & - & - & - & - & - & - & $>$ & - & $>$ & $>$ & - & - & - \\
\hline 137 & - & - & $>$ & $>$ & - & $>$ & $>$ & - & $>$ & - & $>$ & $>$ & - & - & - \\
\hline 138 & - & - & - & - & - & - & - & - & - & - & - & - & - & - & - \\
\hline 139 & - & - & - & - & - & - & - & - & - & - & - & - & - & - & - \\
\hline 140 & - & - & - & - & - & - & - & - & - & - & - & - & - & - & - \\
\hline 141 & - & - & - & - & - & - & - & - & - & - & - & - & - & - & - \\
\hline 142 & - & - & - & - & - & - & - & - & - & - & - & - & - & - & - \\
\hline 143 & - & - & - & - & - & - & - & - & - & - & - & - & - & - & - \\
\hline 144 & - & - & - & - & - & - & - & - & - & - & - & - & - & - & - \\
\hline 145 & - & $<$ & $<$ & - & - & - & - & - & - & - & - & - & - & - & - \\
\hline 146 & - & - & - & - & - & - & - & - & - & - & - & - & - & - & - \\
\hline 147 & - & - & - & - & - & - & - & - & - & - & - & - & - & - & - \\
\hline 148 & - & - & - & - & - & - & - & - & - & - & - & - & - & - & - \\
\hline 149 & - & $<$ & $<$ & - & - & - & - & - & - & - & - & - & - & - & - \\
\hline 166 & - & - & - & - & - & - & - & - & - & - & - & - & - & - & - \\
\hline 167 & - & - & - & - & - & - & - & - & - & - & - & - & - & - & - \\
\hline 168 & - & - & - & - & - & - & - & - & - & - & - & - & - & - & - \\
\hline 169 & - & $<$ & $<$ & $>$ & - & - & - & - & - & - & - & - & - & - & - \\
\hline 170 & - & - & - & $>$ & - & - & - & - & - & - & - & - & - & - & - \\
\hline 171 & - & - & - & - & - & - & - & $>$ & - & - & - & - & - & - & - \\
\hline 172 & - & - & - & - & - & - & - & $>$ & - & - & - & - & - & - & - \\
\hline 173 & - & - & - & - & - & - & - & $>$ & - & - & - & - & - & - & - \\
\hline 174 & $<$ & $<$ & - & - & $<$ & - & - & $"="$ & - & $<$ & - & - & - & - & $<$ \\
\hline 175 & - & - & - & - & - & - & - & $>$ & - & - & - & - & - & - & $<$ \\
\hline 176 & $>$ & - & - & $>$ & $>$ & - & $>$ & $>$ & $>$ & - & $>$ & $>$ & - & $>$ & - \\
\hline 177 & $>$ & - & - & $>$ & $>$ & - & $>$ & $>$ & $>$ & - & $>$ & $>$ & - & - & - \\
\hline 178 & $>$ & - & - & $>$ & $>$ & - & $>$ & $>$ & $>$ & - & $>$ & $>$ & - & $>$ & $>$ \\
\hline 179 & $>$ & $<$ & - & $>$ & $>$ & - & $>$ & $>$ & $>$ & - & $>$ & $>$ & - & - & - \\
\hline 180 & $>$ & - & - & $>$ & $>$ & - & $>$ & $>$ & $>$ & - & $>$ & $>$ & - & - & - \\
\hline 181 & - & - & - & - & - & - & - & - & $>$ & - & - & - & - & - & - \\
\hline 182 & - & - & - & - & - & - & - & - & $>$ & - & - & - & - & - & - \\
\hline 183 & - & - & - & - & - & - & - & - & $>$ & - & - & - & - & - & - \\
\hline 184 & - & - & - & - & - & - & - & - & $>$ & - & - & - & - & - & - \\
\hline 185 & $<$ & $<$ & $<$ & - & - & - & - & - & $"="$ & $<$ & - & $<$ & - & - & - \\
\hline
\end{tabular}


Table 5 (Continued from previous page)

\begin{tabular}{|l|ccccccccccccccc|}
\hline & 48 & 49 & 50 & 51 & 52 & 53 & 54 & 55 & 56 & 57 & 58 & 59 & 60 & 61 & 62 \\
\hline 186 & $>$ & - & - & $>$ & $>$ & - & $>$ & $>$ & $>$ & - & $>$ & $>$ & - & $>$ & $>$ \\
\hline 187 & $>$ & - & - & $>$ & $>$ & - & $>$ & $>$ & $>$ & - & $>$ & $>$ & - & - & - \\
\hline 188 & $>$ & - & - & $>$ & $>$ & - & $>$ & $>$ & $>$ & - & $>$ & $>$ & - & $>$ & $>$ \\
\hline 189 & $>$ & $<$ & - & $>$ & $>$ & - & $>$ & $>$ & $>$ & - & $>$ & $>$ & - & - & - \\
\hline 190 & $>$ & - & - & $>$ & $>$ & - & $>$ & $>$ & $>$ & - & $>$ & $>$ & - & - & - \\
\hline 191 & $>$ & - & - & - & $>$ & - & - & $>$ & $>$ & - & - & - & - & - & - \\
\hline 192 & - & - & - & - & - & - & - & - & - & - & - & - & - & $<$ & - \\
\hline 193 & - & $<$ & $<$ & - & - & - & - & - & - & - & - & - & - & $<$ & - \\
\hline 194 & - & - & - & $>$ & - & - & - & - & - & - & - & - & - & - & - \\
\hline 195 & - & - & - & $>$ & - & - & - & - & - & - & - & - & - & - & - \\
\hline 196 & - & - & - & - & - & - & - & - & - & - & - & - & - & - & - \\
\hline 197 & - & $<$ & $<$ & $>$ & - & - & - & - & - & - & - & - & - & - & - \\
\hline 198 & - & - & - & - & - & - & - & - & - & - & - & - & - & - & - \\
\hline
\end{tabular}

Table 6. Relations between the intuitionistic fuzzy implications $\rightarrow_{63}, \ldots, \rightarrow_{77}$

\begin{tabular}{|c|ccccccccccccccc|}
\hline & 63 & 64 & 65 & 66 & 67 & 68 & 69 & 70 & 71 & 72 & 73 & 74 & 75 & 76 & 77 \\
\hline 1 & - & - & - & - & - & - & - & - & $<$ & - & - & - & - & - & - \\
\hline 2 & - & - & - & - & - & - & - & - & - & - & - & - & - & - & - \\
\hline 3 & - & - & - & - & - & - & - & - & - & - & - & - & - & - & - \\
\hline 4 & - & - & - & - & $>$ & - & - & - & $<$ & $>$ & $>$ & - & - & - & - \\
\hline 5 & - & $>$ & - & - & $>$ & - & - & - & - & $>$ & $>$ & - & - & - & - \\
\hline 6 & - & - & - & - & - & - & - & - & - & - & - & - & - & - & - \\
\hline 7 & - & - & - & - & - & - & - & - & $<$ & - & - & - & - & - & - \\
\hline 8 & - & - & - & - & - & - & - & - & - & - & - & - & - & - & - \\
\hline 9 & - & - & - & - & - & - & - & - & - & - & - & - & - & - & - \\
\hline 10 & - & - & - & - & - & - & - & - & $<$ & - & $>$ & - & - & - & - \\
\hline 11 & - & - & - & - & - & - & - & - & - & - & - & - & - & - & - \\
\hline 12 & - & - & - & - & - & - & - & - & $<$ & $<$ & - & - & - & $<$ & - \\
\hline 13 & - & $>$ & - & - & $>$ & - & - & - & - & $>$ & $>$ & - & - & - & - \\
\hline 14 & - & - & - & - & - & - & - & - & - & - & - & - & - & - & - \\
\hline 15 & $<$ & - & $<$ & - & - & $<$ & $<$ & - & - & - & - & - & - & - & $<$ \\
\hline 16 & - & - & - & - & - & - & - & - & - & - & - & - & - & - & - \\
\hline 17 & - & - & - & - & $>$ & - & - & - & - & $>$ & $>$ & - & - & - & - \\
\hline 18 & - & - & - & - & - & - & - & - & $<$ & - & - & - & - & - & - \\
\hline 19 & - & - & - & - & $<$ & - & - & - & $<$ & - & - & - & $<$ & - & - \\
\hline 20 & - & - & - & - & - & - & - & - & - & - & - & - & - & - & - \\
\hline 21 & - & - & - & - & - & - & - & - & - & - & $>$ & - & - & - & - \\
\hline & & & & & - & - & - & - & - \\
\hline
\end{tabular}


Table 6 (Continued from previous page)

\begin{tabular}{|c|c|c|c|c|c|c|c|c|c|c|c|c|c|c|c|}
\hline & 63 & 64 & 65 & 66 & 67 & 68 & 69 & 70 & 71 & 72 & 73 & 74 & 75 & 76 & 77 \\
\hline 22 & - & - & - & - & - & - & - & - & - & - & - & - & - & - & - \\
\hline 23 & - & - & - & - & - & - & - & - & - & - & - & - & - & - & - \\
\hline 24 & - & - & $<$ & - & - & $<$ & - & - & - & - & - & - & - & - & - \\
\hline 25 & - & - & - & - & - & - & - & - & $<$ & - & - & - & $<$ & - & - \\
\hline 26 & - & - & - & - & - & - & - & - & $<$ & - & - & - & $<$ & - & - \\
\hline 27 & - & - & - & - & - & - & - & - & - & - & - & - & - & - & - \\
\hline 28 & - & - & - & - & - & - & - & - & $<$ & - & - & - & $<$ & - & - \\
\hline 29 & - & - & - & - & - & - & - & - & - & - & - & - & - & - & - \\
\hline 30 & - & - & - & - & - & - & - & - & - & - & - & - & - & - & - \\
\hline 31 & - & - & - & - & - & - & - & - & - & - & - & - & - & - & - \\
\hline 32 & - & - & $>$ & - & - & - & - & - & - & - & - & - & - & - & - \\
\hline 33 & - & - & - & - & $>$ & - & - & $>$ & - & $>$ & $>$ & - & - & $>$ & - \\
\hline 34 & $>$ & $>$ & $>$ & - & $>$ & $>$ & $>$ & $>$ & - & $>$ & $>$ & - & - & $>$ & - \\
\hline 35 & - & - & - & - & - & - & - & - & - & - & - & - & - & - & - \\
\hline 36 & - & - & - & - & - & - & - & - & - & - & - & - & - & - & - \\
\hline 37 & - & - & $>$ & - & - & - & - & - & - & - & - & - & - & - & - \\
\hline 38 & - & - & - & - & - & - & - & - & - & - & - & - & - & - & - \\
\hline 39 & - & - & - & - & - & - & - & $>$ & - & - & $>$ & - & - & - & - \\
\hline 40 & - & - & - & - & - & - & - & - & - & - & - & - & - & - & - \\
\hline 41 & - & - & - & - & - & - & - & - & - & - & - & - & - & - & - \\
\hline 42 & - & - & $>$ & - & - & - & - & - & - & - & - & - & - & - & - \\
\hline 43 & - & - & - & - & - & - & - & - & - & - & - & - & - & - & - \\
\hline 44 & - & - & - & - & - & - & - & - & - & - & - & - & - & - & - \\
\hline 45 & - & - & - & - & - & - & - & - & - & - & - & - & - & - & - \\
\hline 46 & - & - & - & - & - & - & - & - & $<$ & $<$ & - & - & - & $<$ & - \\
\hline 47 & - & - & - & - & - & - & - & - & - & - & - & - & - & - & - \\
\hline 48 & - & - & - & - & - & - & - & - & - & - & - & - & - & - & - \\
\hline 49 & - & - & - & - & - & - & - & - & - & - & - & - & - & - & - \\
\hline 50 & - & - & - & - & - & - & - & - & - & - & - & - & - & - & - \\
\hline 51 & - & - & - & - & - & - & - & - & $<$ & $<$ & - & - & - & $<$ & - \\
\hline 52 & $<$ & - & $<$ & - & - & $<$ & $<$ & - & - & - & - & $<$ & - & - & $<$ \\
\hline 53 & - & - & - & - & - & - & - & - & - & - & - & - & - & - & - \\
\hline 54 & - & - & - & - & - & - & - & - & $<$ & $<$ & - & - & - & $<$ & - \\
\hline 55 & $<$ & - & $<$ & - & - & $<$ & $<$ & - & - & - & - & $<$ & - & - & $<$ \\
\hline 56 & - & - & - & - & $<$ & - & - & - & $<$ & $<$ & - & - & $<$ & $<$ & - \\
\hline 57 & - & - & - & - & - & - & - & - & - & - & - & - & - & - & - \\
\hline
\end{tabular}


Table 6 (Continued from previous page)

\begin{tabular}{|c|c|c|c|c|c|c|c|c|c|c|c|c|c|c|c|}
\hline & 63 & 64 & 65 & 66 & 67 & 68 & 69 & 70 & 71 & 72 & 73 & 74 & 75 & 76 & 77 \\
\hline 58 & - & - & - & - & - & - & - & - & $<$ & $<$ & - & - & $<$ & $<$ & - \\
\hline 59 & - & - & - & - & - & - & - & - & $<$ & $<$ & - & - & $<$ & $<$ & - \\
\hline 60 & - & - & - & - & - & - & - & - & - & - & - & - & - & - & - \\
\hline 61 & - & - & - & - & - & - & - & - & $<$ & - & - & - & - & - & - \\
\hline 62 & $<$ & - & - & - & - & $<$ & - & - & - & - & - & - & - & - & - \\
\hline 63 & $=$ & - & - & - & - & $<$ & - & $>$ & - & - & $>$ & - & - & - & - \\
\hline 64 & - & $=$ & - & - & - & - & - & - & - & - & - & - & - & - & - \\
\hline 65 & - & - & $=$ & - & - & $<$ & - & - & - & - & - & - & - & - & - \\
\hline 66 & - & - & - & $=$ & - & - & - & - & - & - & - & - & - & - & - \\
\hline 67 & - & - & - & - & $=$ & - & - & - & $<$ & - & - & - & - & - & - \\
\hline 68 & $>$ & - & $>$ & - & - & $=$ & $>$ & $>$ & - & - & $>$ & - & - & - & - \\
\hline 69 & - & - & - & - & - & $<$ & $=$ & - & - & - & $>$ & - & - & - & $<$ \\
\hline 70 & $<$ & - & - & - & - & $<$ & - & $=$ & - & - & $>$ & - & - & - & - \\
\hline 71 & - & - & - & - & $>$ & - & - & - & $=$ & $>$ & $>$ & - & - & - & - \\
\hline 72 & - & - & - & - & - & - & - & - & $<$ & $=$ & $>$ & - & - & $<$ & - \\
\hline 73 & $<$ & - & - & - & - & $<$ & $<$ & $<$ & $<$ & $<$ & $=$ & - & - & $<$ & $<$ \\
\hline 74 & - & - & - & - & - & - & - & - & - & - & - & $=$ & - & - & - \\
\hline 75 & - & - & - & - & - & - & - & - & - & - & - & - & $=$ & - & - \\
\hline 76 & - & - & - & - & - & - & - & - & - & $>$ & $>$ & - & - & $=$ & - \\
\hline 77 & - & - & - & - & - & - & $>$ & - & - & - & $>$ & - & - & - & $=$ \\
\hline 78 & $<$ & - & - & - & - & $<$ & - & $<$ & $<$ & - & $>$ & - & - & - & - \\
\hline 79 & - & - & - & - & - & - & - & - & - & - & $>$ & - & - & - & - \\
\hline 80 & - & - & - & - & - & - & - & - & $<$ & - & $>$ & - & - & - & - \\
\hline 81 & - & - & - & - & - & - & - & - & - & - & - & - & - & - & - \\
\hline 82 & - & - & - & - & - & - & - & - & - & - & - & - & - & - & - \\
\hline 83 & - & - & - & - & - & - & - & - & - & - & - & - & - & - & - \\
\hline 84 & $>$ & - & $>$ & - & - & $>$ & $>$ & $>$ & - & - & $>$ & - & - & - & - \\
\hline 85 & - & - & - & - & - & - & - & - & - & - & - & - & - & - & - \\
\hline 86 & - & - & - & - & $>$ & - & - & - & - & - & - & - & - & - & - \\
\hline 87 & - & - & - & - & - & - & - & $>$ & - & - & $>$ & - & - & - & - \\
\hline 88 & $>$ & - & $>$ & - & - & $>$ & $>$ & $>$ & - & - & $>$ & $>$ & - & - & $>$ \\
\hline 89 & - & - & - & - & - & - & - & $>$ & - & - & $>$ & - & - & - & - \\
\hline 90 & - & - & - & - & - & - & - & - & - & - & - & - & - & - & - \\
\hline 91 & - & - & - & - & - & - & - & - & $<$ & $<$ & - & - & - & $<$ & - \\
\hline 92 & $<$ & - & - & - & - & $<$ & $<$ & - & - & - & - & $<$ & - & - & $<$ \\
\hline 93 & $<$ & - & - & - & - & $<$ & $<$ & - & - & - & - & $<$ & - & - & $<$ \\
\hline
\end{tabular}


Table 6 (Continued from previous page)

\begin{tabular}{|c|c|c|c|c|c|c|c|c|c|c|c|c|c|c|c|}
\hline & 63 & 64 & 65 & 66 & 67 & 68 & 69 & 70 & 71 & 72 & 73 & 74 & 75 & 76 & 77 \\
\hline 94 & - & - & - & - & - & - & - & - & - & - & - & - & - & - & - \\
\hline 95 & - & - & - & - & - & - & - & - & $<$ & $<$ & - & - & - & $<$ & - \\
\hline 96 & $<$ & - & - & - & - & $<$ & $<$ & $<$ & $<$ & $<$ & $<$ & $<$ & - & $<$ & $<$ \\
\hline 97 & - & - & - & - & - & - & - & - & - & - & - & $<$ & - & - & $<$ \\
\hline 98 & - & - & - & - & - & - & - & - & $<$ & $<$ & - & - & - & $<$ & - \\
\hline 99 & - & - & - & - & - & - & - & - & - & - & - & - & - & - & - \\
\hline 100 & - & - & - & - & - & - & - & - & - & - & - & - & - & - & - \\
\hline 101 & - & - & - & - & - & - & - & - & - & - & - & - & - & - & - \\
\hline 102 & - & - & - & - & - & - & - & - & - & - & - & - & - & - & - \\
\hline 103 & - & - & - & - & - & - & - & - & - & - & - & - & - & - & - \\
\hline 104 & - & - & - & - & - & - & - & - & - & - & - & - & - & - & - \\
\hline 105 & - & - & - & - & - & - & - & - & - & - & - & - & - & - & - \\
\hline 106 & - & - & - & - & - & - & - & - & - & - & - & - & - & - & - \\
\hline 107 & - & - & - & - & - & - & - & - & - & - & - & - & - & - & - \\
\hline 108 & - & - & - & - & - & - & - & - & - & - & - & - & - & - & - \\
\hline 109 & - & - & - & - & - & - & - & - & - & - & - & - & - & - & - \\
\hline 110 & - & - & - & - & $>$ & - & - & - & - & $>$ & $>$ & - & - & - & - \\
\hline 111 & - & - & - & - & - & - & - & - & - & - & $>$ & - & - & - & - \\
\hline 112 & - & $>$ & - & - & $>$ & - & - & - & - & $>$ & $>$ & - & - & - & - \\
\hline 113 & - & - & - & - & - & - & - & - & - & - & - & - & - & - & - \\
\hline 114 & - & - & - & - & - & - & - & - & - & - & - & - & - & - & - \\
\hline 115 & - & - & - & - & $>$ & - & - & $>$ & - & $>$ & $>$ & - & - & $>$ & - \\
\hline 116 & - & - & - & - & - & - & - & $>$ & - & - & $>$ & - & - & - & - \\
\hline 117 & - & - & - & - & - & - & - & - & - & - & - & - & - & - & - \\
\hline 118 & - & - & - & - & - & - & - & - & - & - & - & - & - & - & - \\
\hline 119 & - & - & - & - & - & - & - & - & - & - & - & - & - & - & - \\
\hline 120 & - & - & - & - & - & - & - & - & - & - & - & - & - & - & - \\
\hline 121 & - & - & - & - & - & - & - & - & - & - & - & - & - & - & - \\
\hline 122 & - & - & - & - & - & - & - & - & - & - & - & - & - & - & - \\
\hline 123 & - & - & - & - & - & - & - & - & - & - & - & - & - & - & - \\
\hline 124 & - & - & - & - & - & - & - & - & - & - & - & - & - & - & - \\
\hline 125 & - & - & - & - & $>$ & - & - & - & $>$ & $>$ & $>$ & - & - & - & - \\
\hline 126 & - & - & - & - & - & - & - & - & - & - & - & - & - & - & - \\
\hline 127 & - & $>$ & - & $>$ & $>$ & - & - & - & $>$ & $>$ & $>$ & - & - & - & - \\
\hline 128 & - & - & - & - & - & - & - & - & - & - & - & - & - & - & - \\
\hline 129 & - & - & - & - & - & - & - & - & - & - & - & - & - & - & - \\
\hline
\end{tabular}


Table 6 (Continued from previous page)

\begin{tabular}{|c|c|c|c|c|c|c|c|c|c|c|c|c|c|c|c|}
\hline & 63 & 64 & 65 & 66 & 67 & 68 & 69 & 70 & 71 & 72 & 73 & 74 & 75 & 76 & 77 \\
\hline 130 & - & - & - & - & $>$ & - & - & $>$ & - & $>$ & $>$ & - & - & $>$ & - \\
\hline 131 & - & - & - & - & - & - & - & - & - & - & - & - & - & - & - \\
\hline 132 & - & - & - & - & - & - & - & - & - & - & - & - & - & - & - \\
\hline 133 & - & - & - & - & - & - & - & - & - & - & - & - & - & - & - \\
\hline 134 & - & - & - & - & - & - & - & - & - & - & - & - & - & - & - \\
\hline 135 & - & - & - & - & - & - & - & - & - & - & - & - & - & - & - \\
\hline 136 & - & - & - & - & - & - & - & - & - & - & - & - & - & - & - \\
\hline 137 & - & - & - & - & - & - & - & - & - & - & - & - & - & - & - \\
\hline 138 & - & - & - & - & - & - & - & - & - & - & - & - & - & - & - \\
\hline 139 & - & - & - & - & - & - & - & - & $<$ & - & - & - & - & - & - \\
\hline 140 & - & - & - & - & - & - & - & - & $<$ & - & - & - & - & - & - \\
\hline 141 & - & - & - & - & - & - & - & - & $<$ & - & - & - & - & - & - \\
\hline 142 & - & - & - & - & - & - & - & - & - & - & - & - & - & - & - \\
\hline 143 & - & - & - & - & - & - & - & - & - & - & - & - & - & $<$ & - \\
\hline 144 & - & - & - & - & - & - & - & - & - & - & - & - & - & - & - \\
\hline 145 & - & - & - & - & - & - & - & - & $<$ & $<$ & - & - & - & $<$ & - \\
\hline 146 & - & - & - & - & - & - & - & - & - & - & - & - & - & - & - \\
\hline 147 & - & - & - & - & - & - & - & - & - & - & - & - & - & $<$ & - \\
\hline 148 & - & - & - & - & - & - & - & - & - & - & - & - & - & - & - \\
\hline 149 & - & - & - & - & - & - & - & - & $<$ & $<$ & - & - & - & $<$ & - \\
\hline 166 & - & - & - & - & - & - & - & - & $<$ & - & - & - & - & - & - \\
\hline 167 & - & - & - & - & - & - & - & - & - & - & - & - & - & $<$ & - \\
\hline 168 & - & - & - & - & - & - & - & - & - & - & - & - & - & - & - \\
\hline 169 & - & - & - & - & - & - & - & - & $<$ & $<$ & - & - & - & $<$ & - \\
\hline 170 & - & - & - & - & - & - & - & - & - & - & - & - & - & - & - \\
\hline 171 & - & - & - & - & - & - & - & - & - & - & - & - & - & - & $<$ \\
\hline 172 & - & - & - & - & - & - & - & - & - & - & - & - & - & - & $<$ \\
\hline 173 & - & - & - & - & - & - & - & - & - & - & - & - & - & - & - \\
\hline 174 & $<$ & - & $<$ & - & - & $<$ & $<$ & - & - & - & - & $<$ & - & - & $<$ \\
\hline 175 & $<$ & - & $<$ & - & - & $<$ & - & - & - & - & - & $<$ & - & - & - \\
\hline 176 & - & - & - & - & $>$ & - & - & - & - & $>$ & $>$ & - & - & - & - \\
\hline 177 & - & - & - & - & - & - & $>$ & - & - & $>$ & $>$ & - & - & $>$ & - \\
\hline 178 & $>$ & - & $>$ & - & $>$ & $>$ & $>$ & $>$ & - & $>$ & $>$ & - & - & $>$ & - \\
\hline 179 & - & - & - & - & - & - & - & - & - & - & - & - & - & - & - \\
\hline 180 & - & - & - & - & - & - & - & - & - & - & - & - & - & - & - \\
\hline 181 & - & - & - & - & - & - & - & - & - & - & - & - & - & - & - \\
\hline
\end{tabular}


Table 6 (Continued from previous page)

\begin{tabular}{|l|lllllllllllllll|}
\hline & 63 & 64 & 65 & 66 & 67 & 68 & 69 & 70 & 71 & 72 & 73 & 74 & 75 & 76 & 77 \\
\hline 182 & - & - & - & - & - & - & - & - & - & - & - & - & - & $<$ & - \\
\hline 183 & - & - & - & - & - & - & - & - & - & - & - & - & - & - & - \\
\hline 184 & - & - & - & - & - & - & - & - & - & - & - & - & - & - & - \\
\hline 185 & - & - & - & - & $<$ & - & - & - & $<$ & $<$ & - & - & $<$ & $<$ & - \\
\hline 186 & $>$ & - & - & - & $>$ & - & - & $>$ & - & $>$ & $>$ & - & - & - & - \\
\hline 187 & - & - & - & - & - & - & - & - & - & - & - & - & - & - & - \\
\hline 188 & $>$ & - & $>$ & - & $>$ & $>$ & $>$ & $>$ & - & $>$ & $>$ & - & - & $>$ & - \\
\hline 189 & - & - & - & - & - & - & - & - & - & - & - & - & - & - & - \\
\hline 190 & - & - & - & - & - & - & - & - & - & - & - & - & - & - & - \\
\hline 191 & - & - & - & - & - & - & - & - & - & - & - & - & - & - & - \\
\hline 192 & - & - & - & - & - & - & - & - & $<$ & - & - & - & - & - & - \\
\hline 193 & - & - & - & - & - & - & - & - & $<$ & $<$ & - & - & - & $<$ & - \\
\hline 194 & - & - & - & - & - & - & - & - & - & - & - & - & - & - & - \\
\hline 195 & - & - & - & - & - & - & - & - & - & - & - & - & - & $<$ & - \\
\hline 196 & - & - & - & - & - & - & - & - & - & - & - & - & - & - & - \\
\hline 197 & - & - & - & - & - & - & - & - & $<$ & $<$ & - & - & - & $<$ & - \\
\hline 198 & - & - & - & - & - & - & - & - & - & - & - & - & - & - & - \\
\hline
\end{tabular}

Table 7. Relations between the intuitionistic fuzzy implications $\rightarrow_{78}, \ldots, \rightarrow_{92}$

\begin{tabular}{|c|ccccccccccccccc|}
\hline & 78 & 79 & 80 & 81 & 82 & 83 & 84 & 85 & 86 & 87 & 88 & 89 & 90 & 91 & 92 \\
\hline 1 & - & - & - & - & - & - & - & - & - & - & - & - & - & - & - \\
\hline 2 & - & - & - & - & - & - & - & - & - & - & - & - & - & - & - \\
\hline 3 & - & - & - & - & - & - & - & - & - & - & - & - & - & - & - \\
\hline 4 & $>$ & - & $>$ & - & - & - & - & - & - & - & - & - & - & $>$ & - \\
\hline 5 & $>$ & - & $>$ & - & - & - & - & - & - & - & - & - & - & $>$ & $>$ \\
\hline 6 & - & - & - & - & - & - & - & - & - & - & - & - & - & - & - \\
\hline 7 & - & - & - & - & $<$ & - & - & - & - & - & - & - & - & - & - \\
\hline 8 & - & - & - & - & - & - & $<$ & - & - & - & $<$ & - & - & - & - \\
\hline 9 & - & - & - & - & - & - & - & - & - & - & - & - & - & - & - \\
\hline 10 & - & - & - & - & - & - & - & - & - & - & - & - & - & - & - \\
\hline 11 & - & - & - & - & - & - & - & - & - & - & - & - & - & - & - \\
\hline 12 & - & - & - & - & - & - & - & - & - & - & - & - & - & $>$ & - \\
\hline 13 & $>$ & - & $>$ & - & - & - & - & - & - & - & - & - & - & $>$ & - \\
\hline 14 & - & - & - & - & - & - & - & - & - & - & - & - & - & - & - \\
\hline 15 & - & - & - & - & - & $<$ & $<$ & - & - & - & $<$ & - & - & - & - \\
\hline 16 & - & - & - & - & - & - & - & - & $<$ & - & - & - & - & - & - \\
\hline & & & & & - & - & - \\
\hline
\end{tabular}


Table 7 (Continued from previous page)

\begin{tabular}{|c|c|c|c|c|c|c|c|c|c|c|c|c|c|c|c|}
\hline & 78 & 79 & 80 & 81 & 82 & 83 & 84 & 85 & 86 & 87 & 88 & 89 & 90 & 91 & 92 \\
\hline 17 & $>$ & - & $>$ & - & - & - & - & - & - & - & - & - & - & $>$ & - \\
\hline 18 & - & - & - & - & - & - & - & - & - & - & - & - & - & $>$ & - \\
\hline 19 & - & - & - & - & - & - & - & - & $<$ & - & - & - & - & - & - \\
\hline 20 & - & - & - & - & - & - & - & - & - & - & - & - & - & - & - \\
\hline 21 & $>$ & - & $>$ & - & - & - & - & - & - & - & - & - & - & - & - \\
\hline 22 & - & - & - & - & - & - & - & - & - & - & - & - & - & $>$ & - \\
\hline 23 & - & - & - & - & - & - & - & - & - & - & - & - & - & - & - \\
\hline 24 & - & - & - & - & - & $<$ & $<$ & - & - & - & $<$ & - & - & - & - \\
\hline 25 & - & - & $<$ & $<$ & - & - & - & - & - & - & - & $<$ & $<$ & - & - \\
\hline 26 & - & - & - & - & - & - & - & - & $<$ & - & - & - & - & - & - \\
\hline 27 & - & - & - & - & - & - & - & - & - & - & - & - & - & - & - \\
\hline 28 & - & - & - & - & - & - & - & - & - & - & - & - & - & - & - \\
\hline 29 & - & - & - & - & - & - & - & - & - & - & - & - & - & - & - \\
\hline 30 & - & - & - & - & - & - & - & - & - & - & - & - & - & - & - \\
\hline 31 & - & - & - & - & - & - & - & - & - & - & - & - & - & - & - \\
\hline 32 & - & - & - & - & - & - & - & - & - & - & - & - & - & - & - \\
\hline 33 & $>$ & - & $>$ & - & $>$ & - & - & - & $>$ & $>$ & - & $>$ & - & $>$ & - \\
\hline 34 & $>$ & - & $>$ & - & $>$ & $>$ & $>$ & $>$ & $>$ & $>$ & - & $>$ & - & $>$ & $>$ \\
\hline 35 & - & - & - & - & - & - & - & - & - & - & - & - & - & - & - \\
\hline 36 & - & - & - & - & $<$ & - & - & - & - & - & - & - & - & - & - \\
\hline 37 & - & - & - & - & - & - & $<$ & - & - & - & $<$ & - & - & - & - \\
\hline 38 & - & - & - & - & - & - & - & - & - & - & - & - & - & - & - \\
\hline 39 & $>$ & - & - & - & - & - & - & - & - & $>$ & - & - & - & - & - \\
\hline 40 & - & - & - & - & - & $<$ & $<$ & - & - & - & $<$ & - & - & - & - \\
\hline 41 & - & - & - & - & - & - & - & - & $<$ & - & - & - & - & - & - \\
\hline 42 & - & - & - & - & - & - & - & - & - & - & - & - & - & - & - \\
\hline 43 & - & - & - & - & - & - & - & - & - & - & - & - & - & - & - \\
\hline 44 & - & - & - & - & - & - & - & - & - & - & - & - & - & - & - \\
\hline 45 & - & - & - & - & - & - & - & - & - & - & - & - & - & - & - \\
\hline 46 & - & - & - & - & - & - & - & - & - & - & - & - & - & - & - \\
\hline 47 & - & - & - & - & - & - & - & - & - & - & - & - & - & - & - \\
\hline 48 & - & - & - & - & - & - & - & - & - & - & - & - & - & - & - \\
\hline 49 & - & - & - & - & - & - & - & - & - & - & - & - & - & $>$ & $>$ \\
\hline 50 & - & - & - & - & - & - & - & - & - & - & - & - & - & $>$ & - \\
\hline 51 & - & - & - & - & - & - & - & - & - & - & - & - & - & $<$ & - \\
\hline 52 & - & $<$ & - & - & - & - & $<$ & - & - & - & $<$ & - & - & - & - \\
\hline 53 & - & - & - & - & - & - & - & - & - & - & - & - & - & - & - \\
\hline
\end{tabular}


Table 7 (Continued from previous page)

\begin{tabular}{|c|c|c|c|c|c|c|c|c|c|c|c|c|c|c|c|}
\hline & 78 & 79 & 80 & 81 & 82 & 83 & 84 & 85 & 86 & 87 & 88 & 89 & 90 & 91 & 92 \\
\hline 54 & - & - & - & - & - & - & - & - & - & - & - & - & - & - & - \\
\hline 55 & - & $<$ & - & - & - & $<$ & $<$ & - & - & - & $<$ & - & - & - & $<$ \\
\hline 56 & - & - & - & - & - & - & - & - & $<$ & - & - & - & - & - & - \\
\hline 57 & - & - & - & - & - & - & - & - & - & - & - & - & - & - & - \\
\hline 58 & - & - & $<$ & $<$ & - & - & - & - & - & - & - & $<$ & $<$ & - & - \\
\hline 59 & - & - & - & - & - & - & - & - & - & - & - & - & - & - & - \\
\hline 60 & - & - & - & - & - & - & - & - & - & - & - & - & - & - & - \\
\hline 61 & - & - & - & - & $<$ & - & - & - & - & - & - & - & - & - & - \\
\hline 62 & - & - & - & - & - & $<$ & $<$ & - & - & - & $<$ & - & - & - & $>$ \\
\hline 63 & $>$ & - & - & - & - & - & $<$ & - & - & - & $<$ & - & - & - & $>$ \\
\hline 64 & - & - & - & - & - & - & - & - & - & - & - & - & - & - & - \\
\hline 65 & - & - & - & - & - & - & $<$ & - & - & - & $<$ & - & - & - & - \\
\hline 66 & - & - & - & - & - & - & - & - & - & - & - & - & - & - & - \\
\hline 67 & - & - & - & - & - & - & - & - & $<$ & - & - & - & - & - & - \\
\hline 68 & $>$ & - & - & - & - & - & $<$ & - & - & - & $<$ & - & - & - & $>$ \\
\hline 69 & - & - & - & - & - & - & $<$ & - & - & - & $<$ & - & - & - & $>$ \\
\hline 70 & $>$ & - & - & - & - & - & $<$ & - & - & $<$ & $<$ & $<$ & - & - & - \\
\hline 71 & $>$ & - & $>$ & - & - & - & - & - & - & - & - & - & - & $>$ & - \\
\hline 72 & - & - & - & - & - & - & - & - & - & - & - & - & - & $>$ & - \\
\hline 73 & $<$ & $<$ & $<$ & - & - & - & $<$ & - & - & $<$ & $<$ & $<$ & - & - & - \\
\hline 74 & - & - & - & - & - & - & - & - & - & - & $<$ & - & - & - & $>$ \\
\hline 75 & - & - & - & - & - & - & - & - & - & - & - & - & - & - & - \\
\hline 76 & - & - & - & - & - & - & - & - & - & - & - & - & - & $>$ & - \\
\hline 77 & - & - & - & - & - & - & - & - & - & - & $<$ & - & - & - & $>$ \\
\hline 78 & $=$ & $<$ & $<$ & - & - & - & $<$ & - & - & $<$ & $<$ & $<$ & - & - & - \\
\hline 79 & $>$ & $=$ & - & - & - & - & - & - & - & - & $<$ & - & - & - & $>$ \\
\hline 80 & $>$ & - & $=$ & - & - & - & - & - & - & - & - & $<$ & - & - & - \\
\hline 81 & - & - & - & $=$ & - & - & - & - & - & - & - & - & $<$ & - & - \\
\hline 82 & - & - & - & - & $=$ & - & - & - & - & - & - & - & - & - & - \\
\hline 83 & - & - & - & - & - & $=$ & $<$ & - & - & - & $<$ & - & - & - & $>$ \\
\hline 84 & $>$ & - & - & - & - & $>$ & $=$ & - & - & $>$ & $<$ & - & - & - & $>$ \\
\hline 85 & - & - & - & - & - & - & - & $=$ & - & - & - & - & - & - & - \\
\hline 86 & - & - & - & - & - & - & - & - & $=$ & - & - & - & - & - & - \\
\hline 87 & $>$ & - & - & - & - & - & $<$ & - & - & $=$ & $<$ & $<$ & - & - & - \\
\hline 88 & $>$ & $>$ & - & - & - & $>$ & $>$ & - & - & $>$ & $=$ & - & - & - & $>$ \\
\hline 89 & $>$ & - & $>$ & - & - & - & - & - & - & $>$ & - & $=$ & - & - & - \\
\hline 90 & - & - & - & $>$ & - & - & - & - & - & - & - & - & $=$ & - & - \\
\hline
\end{tabular}


Table 7 (Continued from previous page)

\begin{tabular}{|c|c|c|c|c|c|c|c|c|c|c|c|c|c|c|c|}
\hline & 78 & 79 & 80 & 81 & 82 & 83 & 84 & 85 & 86 & 87 & 88 & 89 & 90 & 91 & 92 \\
\hline 91 & - & - & - & - & - & - & - & - & - & - & - & - & - & $=$ & - \\
\hline 92 & - & $<$ & - & - & - & $<$ & $<$ & - & - & - & $<$ & - & - & - & $=$ \\
\hline 93 & - & $<$ & - & - & - & - & $<$ & - & - & - & $<$ & - & - & - & $>$ \\
\hline 94 & - & - & - & - & - & - & - & - & - & - & - & - & - & - & - \\
\hline 95 & - & - & - & - & - & - & - & - & - & - & - & - & - & - & - \\
\hline 96 & $<$ & $<$ & $<$ & - & - & - & $<$ & - & - & $<$ & $<$ & $<$ & - & - & - \\
\hline 97 & - & $<$ & - & - & - & - & - & - & - & - & $<$ & - & - & - & $>$ \\
\hline 98 & - & - & $<$ & - & - & - & - & - & - & - & - & $<$ & - & - & - \\
\hline 99 & - & - & - & $<$ & - & - & - & - & - & - & - & - & $<$ & - & - \\
\hline 100 & - & - & - & - & - & - & - & - & - & - & - & - & - & - & - \\
\hline 101 & - & - & - & - & - & - & - & - & - & - & - & - & - & - & - \\
\hline 102 & - & - & - & - & - & - & - & - & - & - & - & - & - & - & - \\
\hline 103 & - & - & - & - & - & - & - & - & - & - & - & - & - & - & - \\
\hline 104 & - & - & - & - & - & - & - & - & - & - & - & - & - & - & - \\
\hline 105 & - & - & - & - & - & - & - & - & - & - & - & - & - & - & - \\
\hline 106 & - & - & - & - & - & - & - & - & - & - & - & - & - & - & - \\
\hline 107 & - & - & - & - & - & - & - & - & - & - & - & - & - & - & - \\
\hline 108 & - & - & - & - & - & - & - & - & - & - & - & - & - & - & - \\
\hline 109 & - & - & - & - & - & - & - & - & - & - & - & - & - & - & - \\
\hline 110 & $>$ & - & $>$ & - & - & - & - & - & - & - & - & - & - & $>$ & - \\
\hline 111 & $>$ & - & $>$ & - & - & - & - & - & - & - & - & - & - & - & - \\
\hline 112 & $>$ & - & $>$ & - & - & - & - & - & - & - & - & - & - & $>$ & - \\
\hline 113 & - & - & - & - & - & - & - & - & - & - & - & - & - & - & - \\
\hline 114 & - & - & - & - & - & - & - & - & - & - & - & - & - & - & - \\
\hline 115 & $>$ & - & $>$ & - & $>$ & - & - & - & $>$ & $>$ & - & $>$ & - & $>$ & - \\
\hline 116 & $>$ & - & $>$ & - & - & - & - & - & - & $>$ & - & $>$ & - & - & - \\
\hline 117 & - & - & - & - & - & - & - & - & - & - & - & - & - & - & - \\
\hline 118 & - & - & - & - & - & - & - & - & - & - & - & - & - & - & - \\
\hline 119 & - & - & - & - & - & - & - & - & - & - & - & - & - & - & - \\
\hline 120 & - & - & - & - & - & - & - & - & - & - & - & - & - & $>$ & - \\
\hline 121 & - & - & - & - & - & - & - & - & - & - & - & - & - & - & - \\
\hline 122 & - & - & - & - & - & - & - & - & - & - & - & - & - & $>$ & - \\
\hline 123 & - & - & - & - & - & - & - & - & - & - & - & - & - & - & - \\
\hline 124 & - & - & - & - & - & - & - & - & - & - & - & - & - & - & - \\
\hline 125 & $>$ & - & $>$ & - & - & - & - & - & - & - & - & - & - & $>$ & - \\
\hline 126 & - & - & - & - & - & - & - & - & - & - & - & - & - & - & - \\
\hline 127 & $>$ & - & $>$ & - & - & - & - & - & - & - & - & - & - & $>$ & - \\
\hline \multicolumn{16}{|c|}{ (Continued on next page) } \\
\hline
\end{tabular}


Table 7 (Continued from previous page)

\begin{tabular}{|c|c|c|c|c|c|c|c|c|c|c|c|c|c|c|c|}
\hline & 78 & 79 & 80 & 81 & 82 & 83 & 84 & 85 & 86 & 87 & 88 & 89 & 90 & 91 & 92 \\
\hline 128 & - & - & - & - & - & - & - & - & - & - & - & - & - & - & - \\
\hline 129 & - & - & - & - & - & - & - & - & - & - & - & - & - & - & - \\
\hline 130 & $>$ & - & $>$ & - & $>$ & - & - & - & $>$ & $>$ & - & $>$ & - & $>$ & - \\
\hline 131 & - & - & - & - & - & - & - & - & - & - & - & - & - & - & - \\
\hline 132 & - & - & - & - & - & - & - & - & - & - & - & - & - & - & - \\
\hline 133 & - & - & - & - & - & - & - & - & - & - & - & - & - & - & - \\
\hline 134 & - & - & - & - & - & - & - & - & - & - & - & - & - & - & - \\
\hline 135 & - & - & - & - & - & - & - & - & - & - & - & - & - & $>$ & - \\
\hline 136 & - & - & - & - & - & - & - & - & - & - & - & - & - & - & - \\
\hline 137 & - & - & - & - & - & - & - & - & - & - & - & - & - & $>$ & - \\
\hline 138 & - & - & - & - & - & - & - & - & - & - & - & - & - & - & - \\
\hline 139 & - & - & - & - & - & - & - & - & - & - & - & - & - & - & - \\
\hline 140 & - & - & - & - & - & - & - & - & - & - & - & - & - & - & - \\
\hline 141 & - & - & - & - & - & - & - & - & - & - & - & - & - & - & - \\
\hline 142 & - & - & - & - & - & - & - & - & - & - & - & - & - & - & - \\
\hline 143 & - & - & - & - & - & - & - & - & - & - & - & - & - & - & - \\
\hline 144 & - & - & - & - & - & - & - & - & - & - & - & - & - & - & - \\
\hline 145 & - & - & - & - & - & - & - & - & - & - & - & - & - & - & - \\
\hline 146 & - & - & - & - & - & - & - & - & - & - & - & - & - & - & - \\
\hline 147 & - & - & - & - & - & - & - & - & - & - & - & - & - & - & - \\
\hline 148 & - & - & - & - & - & - & - & - & - & - & - & - & - & - & - \\
\hline 149 & - & - & - & - & - & - & - & - & - & - & - & - & - & - & - \\
\hline 166 & - & - & - & - & - & - & - & - & - & - & - & - & - & - & - \\
\hline 167 & - & - & - & - & - & - & - & - & - & - & - & - & - & - & - \\
\hline 168 & - & - & - & - & - & - & - & - & - & - & - & - & - & - & - \\
\hline 169 & - & - & - & - & - & - & - & - & - & - & - & - & - & - & - \\
\hline 170 & - & - & - & - & - & - & - & - & - & - & - & - & - & - & - \\
\hline 171 & - & - & - & - & - & $<$ & $<$ & - & - & - & $<$ & - & - & - & - \\
\hline 172 & - & - & - & - & - & $<$ & $<$ & - & - & - & $<$ & - & - & - & - \\
\hline 173 & - & - & - & - & - & $<$ & $<$ & - & - & - & $<$ & - & - & - & - \\
\hline 174 & - & $<$ & - & - & - & $<$ & $<$ & - & - & - & $<$ & - & - & - & $<$ \\
\hline 175 & - & - & - & - & - & $<$ & $<$ & - & - & - & $<$ & - & - & - & - \\
\hline 176 & $>$ & - & $>$ & - & - & - & - & - & - & - & - & - & - & $>$ & $>$ \\
\hline 177 & - & - & - & - & - & - & - & - & - & - & - & - & - & $>$ & $>$ \\
\hline 178 & $>$ & - & $>$ & - & $>$ & $>$ & $>$ & - & $>$ & $>$ & - & $>$ & - & $>$ & $>$ \\
\hline 179 & - & - & - & - & - & - & - & - & - & - & - & - & - & $>$ & $>$ \\
\hline 180 & - & - & - & - & - & - & - & - & - & - & - & - & - & $>$ & $>$ \\
\hline \multicolumn{16}{|c|}{ (Continued on next page) } \\
\hline
\end{tabular}


Table 7 (Continued from previous page)

\begin{tabular}{|c|ccccccccccccccc|}
\hline & 78 & 79 & 80 & 81 & 82 & 83 & 84 & 85 & 86 & 87 & 88 & 89 & 90 & 91 & 92 \\
\hline 181 & - & - & - & - & - & - & - & - & $<$ & - & - & - & - & - & - \\
\hline 182 & - & - & - & - & - & - & - & - & $<$ & - & - & - & - & - & - \\
\hline 183 & - & - & - & - & - & - & - & - & $<$ & - & - & - & - & - & - \\
\hline 184 & - & - & - & - & - & - & - & - & $<$ & - & - & - & - & - & - \\
\hline 185 & - & - & - & - & - & - & - & - & $<$ & - & - & - & - & - & - \\
\hline 186 & $>$ & - & $>$ & - & - & - & - & - & - & - & - & - & - & $>$ & $>$ \\
\hline 187 & - & - & - & - & - & - & - & - & - & - & - & - & - & $>$ & $>$ \\
\hline 188 & $>$ & - & $>$ & - & $>$ & $>$ & $>$ & - & $>$ & $>$ & - & $>$ & - & $>$ & $>$ \\
\hline 189 & - & - & - & - & - & - & - & - & - & - & - & - & - & $>$ & $>$ \\
\hline 190 & - & - & - & - & - & - & - & - & - & - & - & - & - & $>$ & $>$ \\
\hline 191 & - & - & - & - & - & - & - & - & - & - & - & - & - & - & - \\
\hline 192 & - & - & - & - & $<$ & - & - & - & - & - & - & - & - & - & - \\
\hline 193 & - & - & - & - & $<$ & - & - & - & - & - & - & - & - & $<$ & - \\
\hline 194 & - & - & - & - & - & - & - & - & - & - & - & - & - & - & - \\
\hline 195 & - & - & - & - & - & - & - & - & - & - & - & - & - & - & - \\
\hline 196 & - & - & - & - & $<$ & - & - & - & - & - & - & - & - & - & - \\
\hline 197 & - & - & - & - & - & - & - & - & - & - & - & - & - & $<$ & - \\
\hline 198 & - & - & - & - & $<$ & - & - & - & - & - & - & - & - & - & - \\
\hline
\end{tabular}

Table 8. Relations between the intuitionistic fuzzy implications $\rightarrow_{93}, \ldots, \rightarrow_{107}$

\begin{tabular}{|l|ccccccccccccccc|}
\hline & 93 & 94 & 95 & 96 & 97 & 98 & 99 & 100 & 101 & 102 & 103 & 104 & 105 & 106 & 107 \\
\hline 1 & - & - & - & - & - & - & - & - & - & - & - & - & - & - & - \\
\hline 2 & - & - & - & - & - & - & - & - & - & - & - & - & - & - & - \\
\hline 3 & - & - & - & - & - & - & - & - & - & - & - & - & - & - & - \\
\hline 4 & - & - & $>$ & $>$ & - & $>$ & - & - & - & - & - & - & - & - & - \\
\hline 5 & $>$ & $>$ & $>$ & $>$ & - & $>$ & - & - & - & - & - & - & - & - & - \\
\hline 6 & - & - & - & - & - & - & - & - & - & - & - & - & - & - & - \\
\hline 7 & - & - & - & - & - & - & - & - & - & - & - & - & - & - & - \\
\hline 8 & - & - & - & - & - & - & - & - & - & - & - & - & - & - & - \\
\hline 9 & - & - & - & - & - & - & - & - & - & - & - & - & - & - & - \\
\hline 10 & - & - & - & $>$ & - & - & - & - & - & - & - & - & - & - & - \\
\hline 11 & - & - & - & - & - & - & - & - & - & - & - & - & - & - & - \\
\hline 12 & - & - & $>$ & $>$ & - & $>$ & - & - & - & - & - & - & - & - & - \\
\hline 13 & - & $>$ & $>$ & $>$ & - & $>$ & - & - & - & - & - & - & - & - & - \\
\hline 14 & - & - & - & - & - & - & - & - & - & - & - & - & - & - & - \\
\hline 15 & - & - & - & - & - & - & - & - & - & - & - & - & - & - & - \\
\hline 16 & - & - & - & - & - & - & - & - & - & - & - & - & - & - & - \\
\hline
\end{tabular}

(Continued on next page) 
Table 8 (Continued from previous page)

\begin{tabular}{|c|c|c|c|c|c|c|c|c|c|c|c|c|c|c|c|}
\hline & 93 & 94 & 95 & 96 & 97 & 98 & 99 & 100 & 101 & 102 & 103 & 104 & 105 & 106 & 107 \\
\hline 17 & - & - & $>$ & $>$ & - & $>$ & - & - & - & - & - & - & - & - & - \\
\hline 18 & - & - & $>$ & $>$ & - & $>$ & - & - & - & - & - & - & - & - & - \\
\hline 19 & - & - & - & - & - & - & - & - & - & - & - & - & - & - & - \\
\hline 20 & - & - & - & - & - & - & - & - & - & - & - & - & - & - & - \\
\hline 21 & - & - & - & $>$ & - & $>$ & - & - & - & - & - & - & - & - & - \\
\hline 22 & - & - & $>$ & $>$ & - & $>$ & - & - & - & - & - & - & - & - & - \\
\hline 23 & - & - & - & - & - & - & - & - & - & - & - & - & - & - & - \\
\hline 24 & - & - & - & - & - & - & - & - & - & - & - & - & - & - & - \\
\hline 25 & - & - & - & - & - & - & - & - & - & - & - & - & - & - & - \\
\hline 26 & - & - & - & - & - & - & - & - & - & - & - & - & - & - & - \\
\hline 27 & - & - & - & - & - & - & - & - & - & - & - & - & - & - & - \\
\hline 28 & - & - & - & - & - & - & - & - & - & - & - & - & - & - & - \\
\hline 29 & - & - & - & - & - & - & - & - & - & - & - & - & - & - & - \\
\hline 30 & - & - & - & - & - & - & - & - & - & - & - & - & - & - & - \\
\hline 31 & - & - & - & - & - & - & - & - & - & - & - & - & - & - & - \\
\hline 32 & - & - & - & - & - & - & - & - & - & - & - & - & - & - & - \\
\hline 33 & - & - & $>$ & $>$ & - & $>$ & - & - & - & - & - & - & - & - & - \\
\hline 34 & $>$ & $>$ & $>$ & $>$ & - & $>$ & - & - & - & - & - & - & - & - & - \\
\hline 35 & - & - & - & - & - & - & - & - & - & - & - & - & - & - & - \\
\hline 36 & - & - & - & - & - & - & - & - & - & - & - & - & - & - & - \\
\hline 37 & - & - & - & - & - & - & - & - & - & - & - & - & - & - & - \\
\hline 38 & - & - & - & - & - & - & - & - & - & - & - & - & - & - & - \\
\hline 39 & - & - & - & $>$ & - & - & - & - & - & - & - & - & - & - & - \\
\hline 40 & - & - & - & - & - & - & - & - & - & - & - & - & - & - & - \\
\hline 41 & - & - & - & - & - & - & - & - & - & - & - & - & - & - & - \\
\hline 42 & - & - & - & - & - & - & - & - & - & - & - & - & - & - & - \\
\hline 43 & - & - & - & - & - & - & - & - & - & - & - & - & - & - & - \\
\hline 44 & - & - & - & - & - & - & - & - & - & - & - & - & - & - & - \\
\hline 45 & - & - & - & - & - & - & - & - & - & - & - & - & - & - & - \\
\hline 46 & - & - & - & - & - & - & - & - & - & - & - & - & - & - & - \\
\hline 47 & - & - & - & - & - & - & - & - & - & - & - & - & - & - & - \\
\hline 48 & - & - & - & - & - & - & - & - & - & - & - & - & - & - & - \\
\hline 49 & $>$ & $>$ & $>$ & $>$ & - & $>$ & - & - & - & - & - & - & - & - & - \\
\hline 50 & - & $>$ & $>$ & $>$ & - & $>$ & - & - & - & - & - & - & - & - & - \\
\hline 51 & - & - & - & - & - & - & - & - & - & - & - & - & - & - & - \\
\hline 52 & $<$ & - & - & - & $<$ & - & - & - & - & - & - & - & - & - & - \\
\hline 53 & - & - & - & - & - & - & - & - & - & - & - & - & - & - & - \\
\hline
\end{tabular}

(Continued on next page) 
Table 8 (Continued from previous page)

\begin{tabular}{|c|c|c|c|c|c|c|c|c|c|c|c|c|c|c|c|}
\hline & 93 & 94 & 95 & 96 & 97 & 98 & 99 & 100 & 101 & 102 & 103 & 104 & 105 & 106 & 107 \\
\hline 54 & - & - & - & $>$ & - & - & - & - & - & - & - & - & - & - & - \\
\hline 55 & $<$ & - & - & - & $<$ & - & - & - & - & - & - & - & - & - & - \\
\hline 56 & - & - & $<$ & - & - & - & - & - & - & - & - & - & - & - & - \\
\hline 57 & - & - & - & - & - & - & - & - & - & - & - & - & - & - & - \\
\hline 58 & - & - & - & - & - & $<$ & $<$ & - & - & - & - & - & - & - & - \\
\hline 59 & - & - & - & - & - & - & - & - & - & - & - & - & - & - & - \\
\hline 60 & - & - & - & - & - & - & - & - & - & - & - & - & - & - & - \\
\hline 61 & - & - & - & - & - & - & - & - & - & - & - & - & - & - & - \\
\hline 62 & - & - & - & - & - & - & - & - & - & - & - & - & - & - & - \\
\hline 63 & $>$ & - & - & $>$ & - & - & - & - & - & - & - & - & - & - & - \\
\hline 64 & - & - & - & - & - & - & - & - & - & - & - & - & - & - & - \\
\hline 65 & - & - & - & - & - & - & - & - & - & - & - & - & - & - & - \\
\hline 66 & - & - & - & - & - & - & - & - & - & - & - & - & - & - & - \\
\hline 67 & - & - & - & - & - & - & - & - & - & - & - & - & - & - & - \\
\hline 68 & $>$ & - & - & $>$ & - & - & - & - & - & - & - & - & - & - & - \\
\hline 69 & $>$ & - & - & $>$ & - & - & - & - & - & - & - & - & - & - & - \\
\hline 70 & - & - & - & $>$ & - & - & - & - & - & - & - & - & - & - & - \\
\hline 71 & - & - & $>$ & $>$ & - & $>$ & - & - & - & - & - & - & - & - & - \\
\hline 72 & - & - & $>$ & $>$ & - & $>$ & - & - & - & - & - & - & - & - & - \\
\hline 73 & - & - & - & $>$ & - & - & - & - & - & - & - & - & - & - & - \\
\hline 74 & $>$ & - & - & $>$ & $>$ & - & - & - & - & - & - & - & - & - & - \\
\hline 75 & - & - & - & - & - & - & - & - & - & - & - & - & - & - & - \\
\hline 76 & - & - & $>$ & $>$ & - & $>$ & - & - & - & - & - & - & - & - & - \\
\hline 77 & $>$ & - & - & $>$ & $>$ & - & - & - & - & - & - & - & - & - & - \\
\hline 78 & - & - & - & $>$ & - & - & - & - & - & - & - & - & - & - & - \\
\hline 79 & $>$ & - & - & $>$ & $>$ & - & - & - & - & - & - & - & - & - & - \\
\hline 80 & - & - & - & $>$ & - & $>$ & - & - & - & - & - & - & - & - & - \\
\hline 81 & - & - & - & - & - & - & $>$ & - & - & - & - & - & - & - & - \\
\hline 82 & - & - & - & - & - & - & - & - & - & - & - & - & - & - & - \\
\hline 83 & - & - & - & - & - & - & - & - & - & - & - & - & - & - & - \\
\hline 84 & $>$ & - & - & $>$ & - & - & - & - & - & - & - & - & - & - & - \\
\hline 85 & - & - & - & - & - & - & - & - & - & - & - & - & - & - & - \\
\hline 86 & - & - & - & - & - & - & - & - & - & - & - & - & - & - & - \\
\hline 87 & - & - & - & $>$ & - & - & - & - & - & - & - & - & - & - & - \\
\hline 88 & $>$ & - & - & $>$ & $>$ & - & - & - & - & - & - & - & - & - & - \\
\hline 89 & - & - & - & $>$ & - & $>$ & - & - & - & - & - & - & - & - & - \\
\hline 90 & - & - & - & - & - & - & $>$ & - & - & - & - & - & - & - & - \\
\hline
\end{tabular}

(Continued on next page) 
Table 8 (Continued from previous page)

\begin{tabular}{|c|c|c|c|c|c|c|c|c|c|c|c|c|c|c|c|}
\hline & 93 & 94 & 95 & 96 & 97 & 98 & 99 & 100 & 101 & 102 & 103 & 104 & 105 & 106 & 107 \\
\hline 91 & - & - & - & - & - & - & - & - & - & - & - & - & - & - & - \\
\hline 92 & $<$ & - & - & - & $<$ & - & - & - & - & - & - & - & - & - & - \\
\hline 93 & $=$ & - & - & $>$ & $<$ & - & - & - & - & - & - & - & - & - & - \\
\hline 94 & - & $=$ & - & - & - & - & - & - & - & - & - & - & - & - & - \\
\hline 95 & - & - & $=$ & - & - & - & - & - & - & - & - & - & - & - & - \\
\hline 96 & $<$ & - & - & $=$ & $<$ & $<$ & - & - & - & - & - & - & - & - & - \\
\hline 97 & $>$ & - & - & $>$ & $=$ & - & - & - & - & - & - & - & - & - & - \\
\hline 98 & - & - & - & $>$ & - & $=$ & - & - & - & - & - & - & - & - & - \\
\hline 99 & - & - & - & - & - & - & $=$ & - & - & - & - & - & - & - & - \\
\hline 100 & - & - & - & - & - & - & - & $=$ & - & - & - & - & - & - & - \\
\hline 101 & - & - & - & - & - & - & - & - & $=$ & - & - & - & - & - & - \\
\hline 102 & - & - & - & - & - & - & - & - & - & $=$ & - & - & - & - & - \\
\hline 103 & - & - & - & - & - & - & - & - & - & - & $=$ & - & - & - & - \\
\hline 104 & - & - & - & - & - & - & - & - & - & - & - & $=$ & - & - & - \\
\hline 105 & - & - & - & - & - & - & - & - & - & - & - & - & $=$ & - & - \\
\hline 106 & - & - & - & - & - & - & - & - & - & - & - & - & - & $=$ & - \\
\hline 107 & - & - & - & - & - & - & - & - & - & - & - & - & - & - & $=$ \\
\hline 108 & - & - & - & - & - & - & - & - & - & - & - & - & - & - & - \\
\hline 109 & - & - & - & - & - & - & - & - & - & - & - & - & - & - & - \\
\hline 110 & - & - & $>$ & $>$ & - & $>$ & - & - & - & - & - & - & - & - & - \\
\hline 111 & - & - & - & $>$ & - & $>$ & - & - & - & - & - & - & - & - & - \\
\hline 112 & - & $>$ & $>$ & $>$ & - & $>$ & - & - & - & - & - & - & - & - & - \\
\hline 113 & - & - & - & - & - & - & - & - & - & - & - & - & - & - & - \\
\hline 114 & - & - & - & - & - & - & - & - & - & - & - & - & - & - & - \\
\hline 115 & - & - & $>$ & $>$ & - & $>$ & - & - & - & - & - & - & - & - & - \\
\hline 116 & - & - & - & $>$ & - & $>$ & - & - & - & - & - & - & - & - & - \\
\hline 117 & - & - & - & - & - & - & - & - & - & - & - & - & - & - & - \\
\hline 118 & - & - & - & - & - & - & - & - & - & - & - & - & - & - & - \\
\hline 119 & - & - & - & - & - & - & - & - & - & - & - & - & - & - & - \\
\hline 120 & - & - & $>$ & $>$ & - & $>$ & - & - & - & - & - & - & - & - & - \\
\hline 121 & - & - & - & $>$ & - & $>$ & - & - & - & - & - & - & - & - & - \\
\hline 122 & - & $>$ & $>$ & $>$ & - & $>$ & - & - & - & - & - & - & - & - & - \\
\hline 123 & - & - & - & - & - & - & - & - & - & - & - & - & - & - & - \\
\hline 124 & - & - & - & - & - & - & - & - & - & - & - & - & - & - & - \\
\hline 125 & - & - & $>$ & $>$ & - & $>$ & - & - & - & - & - & - & - & - & - \\
\hline 126 & - & - & - & - & - & - & - & - & - & - & - & - & - & - & - \\
\hline 127 & - & $>$ & $>$ & $>$ & - & $>$ & - & - & - & - & - & - & - & - & - \\
\hline
\end{tabular}

(Continued on next page) 
Table 8 (Continued from previous page)

\begin{tabular}{|c|c|c|c|c|c|c|c|c|c|c|c|c|c|c|c|}
\hline & 93 & 94 & 95 & 96 & 97 & 98 & 99 & 100 & 101 & 102 & 103 & 104 & 105 & 106 & 107 \\
\hline 128 & - & - & - & - & - & - & - & - & - & - & - & - & - & - & - \\
\hline 129 & - & - & - & - & - & - & - & - & - & - & - & - & - & - & - \\
\hline 130 & - & - & $>$ & $>$ & - & $>$ & - & - & - & - & - & - & - & - & - \\
\hline 131 & - & - & - & - & - & - & - & - & - & - & - & - & - & - & - \\
\hline 132 & - & - & - & - & - & - & - & - & - & - & - & - & - & - & - \\
\hline 133 & - & - & - & - & - & - & - & - & - & - & - & - & - & - & - \\
\hline 134 & - & - & - & - & - & - & - & - & - & - & - & - & - & - & - \\
\hline 135 & - & - & $>$ & $>$ & - & $>$ & - & - & - & - & - & - & - & - & - \\
\hline 136 & - & - & - & - & - & - & - & - & - & - & - & - & - & - & - \\
\hline 137 & - & $>$ & $>$ & $>$ & - & $>$ & - & - & - & - & - & - & - & - & - \\
\hline 138 & - & - & - & - & - & - & - & - & - & - & - & - & - & - & - \\
\hline 139 & - & - & - & - & - & - & - & - & - & - & - & - & - & - & - \\
\hline 140 & - & - & - & - & - & - & - & - & - & - & - & - & - & - & - \\
\hline 141 & - & - & - & - & - & - & - & - & - & - & - & - & - & - & - \\
\hline 142 & - & - & - & - & - & - & - & - & - & - & - & - & - & - & - \\
\hline 143 & - & - & - & - & - & - & - & - & - & - & - & - & - & - & - \\
\hline 144 & - & - & - & - & - & - & - & - & - & - & - & - & - & - & - \\
\hline 145 & - & - & - & - & - & - & - & - & - & - & - & - & - & - & - \\
\hline 146 & - & - & - & - & - & - & - & - & - & - & - & - & - & - & - \\
\hline 147 & - & - & - & - & - & - & - & - & - & - & - & - & - & - & - \\
\hline 148 & - & - & - & - & - & - & - & - & - & - & - & - & - & - & - \\
\hline 149 & - & - & - & - & - & - & - & - & - & - & - & - & - & - & - \\
\hline 166 & - & - & - & - & - & - & - & - & - & - & - & - & - & - & - \\
\hline 167 & - & - & - & - & - & - & - & - & - & - & - & - & - & - & - \\
\hline 168 & - & - & - & - & - & - & - & - & - & - & - & - & - & - & - \\
\hline 169 & - & - & - & - & - & - & - & - & - & - & - & - & - & - & - \\
\hline 170 & - & - & - & - & - & - & - & - & - & - & - & - & - & - & - \\
\hline 171 & - & - & - & - & - & - & - & - & - & - & - & - & - & - & - \\
\hline 172 & - & - & - & - & - & - & - & - & - & - & - & - & - & - & - \\
\hline 173 & - & - & - & - & - & - & - & - & - & - & - & - & - & - & - \\
\hline 174 & $<$ & - & - & - & $<$ & - & - & - & - & - & - & - & - & - & - \\
\hline 175 & - & - & - & - & - & - & - & - & - & - & - & - & - & - & - \\
\hline 176 & $>$ & - & $>$ & $>$ & - & $>$ & - & - & - & - & - & - & - & - & - \\
\hline 177 & $>$ & - & $>$ & $>$ & - & $>$ & - & - & - & - & - & - & - & - & - \\
\hline 178 & $>$ & - & $>$ & $>$ & - & $>$ & - & - & - & - & - & - & - & - & - \\
\hline 179 & $>$ & - & $>$ & $>$ & - & $>$ & - & - & - & - & - & - & - & - & - \\
\hline 180 & $>$ & - & $>$ & $>$ & - & $>$ & - & - & - & - & - & - & - & - & - \\
\hline
\end{tabular}

(Continued on next page) 
Table 8 (Continued from previous page)

\begin{tabular}{|l|ccccccccccccccc|}
\hline & 93 & 94 & 95 & 96 & 97 & 98 & 99 & 100 & 101 & 102 & 103 & 104 & 105 & 106 & 107 \\
\hline 181 & - & - & - & - & - & - & - & - & - & - & - & - & - & - & - \\
\hline 182 & - & - & - & - & - & - & - & - & - & - & - & - & - & - & - \\
\hline 183 & - & - & - & - & - & - & - & - & - & - & - & - & - & - & - \\
\hline 184 & - & - & - & - & - & - & - & - & - & - & - & - & - & - & - \\
\hline 185 & - & - & $<$ & - & - & - & - & - & - & - & - & - & - & - & - \\
\hline 186 & $>$ & - & $>$ & $>$ & - & $>$ & - & - & - & - & - & - & - & - & - \\
\hline 187 & $>$ & - & $>$ & $>$ & - & $>$ & - & - & - & - & - & - & - & - & - \\
\hline 188 & $>$ & - & $>$ & $>$ & - & $>$ & - & - & - & - & - & - & - & - & - \\
\hline 189 & $>$ & - & $>$ & $>$ & - & $>$ & - & - & - & - & - & - & - & - & - \\
\hline 190 & $>$ & - & $>$ & $>$ & - & $>$ & - & - & - & - & - & - & - & - & - \\
\hline 191 & - & - & - & - & - & - & - & - & - & - & - & - & - & - & - \\
\hline 192 & - & - & - & - & - & - & - & - & - & - & - & - & - & - & - \\
\hline 193 & - & - & - & - & - & - & - & - & - & - & - & - & - & - & - \\
\hline 194 & - & - & - & - & - & - & - & - & - & - & - & - & - & - & - \\
\hline 195 & - & - & - & - & - & - & - & - & - & - & - & - & - & - & - \\
\hline 196 & - & - & - & - & - & - & - & - & - & - & - & - & - & - & - \\
\hline 197 & - & - & - & - & - & - & - & - & - & - & - & - & - & - & - \\
\hline 198 & - & - & - & - & - & - & - & - & - & - & - & - & - & - & - \\
\hline
\end{tabular}

Table 9. Relations between the intuitionistic fuzzy implications $\rightarrow_{108}, \ldots, \rightarrow_{122}$

\begin{tabular}{|c|ccccccccccccccc|}
\hline & 108 & 109 & 110 & 111 & 112 & 113 & 114 & 115 & 116 & 117 & 118 & 119 & 120 & 121 & 122 \\
\hline 1 & - & - & $<$ & - & $<$ & - & - & $<$ & - & - & - & - & - & - & - \\
\hline 2 & - & - & - & - & - & - & - & - & - & - & - & - & - & - & - \\
\hline 3 & - & - & - & - & - & - & - & - & - & - & - & - & - & - & - \\
\hline 4 & - & - & $<$ & - & $<$ & - & - & $<$ & - & - & - & - & - & - & - \\
\hline 5 & - & - & - & - & - & - & - & - & - & - & - & - & - & - & - \\
\hline 6 & - & - & - & - & $<$ & - & - & - & - & - & - & - & - & - & - \\
\hline 7 & - & - & $<$ & - & $<$ & - & - & $<$ & - & - & - & - & - & - & - \\
\hline 8 & - & - & - & - & - & - & - & - & - & - & - & - & - & - & - \\
\hline 9 & - & - & - & - & $<$ & - & - & - & - & - & - & - & - & - & - \\
\hline 10 & - & - & $<$ & - & $<$ & - & - & $<$ & - & - & - & - & - & - & - \\
\hline 11 & - & - & - & - & - & - & - & - & - & - & - & - & - & - & - \\
\hline 12 & - & - & $<$ & - & $<$ & - & - & $<$ & - & - & - & - & $<$ & - & $<$ \\
\hline 13 & - & - & - & - & $<$ & - & - & - & - & - & - & - & - & - & - \\
\hline 14 & - & - & - & - & - & - & - & - & - & - & - & - & - & - & - \\
\hline 15 & - & - & - & - & - & - & - & - & - & - & - & - & - & - & - \\
\hline 16 & - & - & - & - & - & - & - & $<$ & - & - & - & - & - & - & - \\
\hline
\end{tabular}

(Continued on next page) 
Table 9 (Continued from previous page)

\begin{tabular}{|c|c|c|c|c|c|c|c|c|c|c|c|c|c|c|c|}
\hline & 108 & 109 & 110 & 111 & 112 & 113 & 114 & 115 & 116 & 117 & 118 & 119 & 120 & 121 & 122 \\
\hline 17 & - & - & $<$ & - & $<$ & - & - & - & - & - & - & - & - & - & - \\
\hline 18 & - & - & $<$ & - & $<$ & - & - & $<$ & - & - & - & - & - & - & - \\
\hline 19 & - & - & $<$ & - & $<$ & - & - & $<$ & - & - & - & - & - & - & - \\
\hline 20 & - & - & - & - & - & - & - & - & - & - & - & - & - & - & - \\
\hline 21 & - & - & - & - & - & - & - & - & - & - & - & - & - & - & - \\
\hline 22 & - & - & - & - & - & - & - & $<$ & - & - & - & - & - & - & - \\
\hline 23 & - & - & - & - & - & - & - & - & - & - & - & - & - & - & - \\
\hline 24 & - & - & - & - & - & - & - & - & - & - & - & - & - & - & - \\
\hline 25 & - & - & $<$ & $<$ & $<$ & - & - & $<$ & $<$ & - & - & - & - & - & - \\
\hline 26 & - & - & $<$ & - & $<$ & - & - & $<$ & - & - & - & - & - & - & - \\
\hline 27 & - & - & - & - & - & - & - & - & - & - & - & - & - & - & - \\
\hline 28 & - & - & $<$ & - & $<$ & - & - & $<$ & - & - & - & - & - & - & - \\
\hline 29 & - & - & - & - & - & - & - & - & - & - & - & - & - & - & - \\
\hline 30 & - & - & - & - & - & - & - & $<$ & - & - & - & - & - & - & - \\
\hline 31 & - & - & - & - & - & - & - & - & - & - & - & - & - & - & - \\
\hline 32 & - & - & - & - & - & - & - & - & - & - & - & - & - & - & - \\
\hline 33 & - & - & - & - & - & - & - & $<$ & - & - & - & - & - & - & - \\
\hline 34 & - & - & - & - & - & - & - & - & - & - & - & - & - & - & - \\
\hline 35 & - & - & - & - & - & - & - & - & $<$ & - & - & - & - & - & - \\
\hline 36 & - & - & - & - & - & - & - & $<$ & - & - & - & - & - & - & - \\
\hline 37 & - & - & - & - & - & - & - & - & - & - & - & - & - & - & - \\
\hline 38 & - & - & - & - & - & - & - & - & - & - & - & - & - & - & - \\
\hline 39 & - & - & - & - & - & - & - & $<$ & - & - & - & - & - & - & - \\
\hline 40 & - & - & - & - & - & - & - & - & - & - & - & - & - & - & - \\
\hline 41 & - & - & - & - & - & - & - & $<$ & - & - & - & - & - & - & - \\
\hline 42 & - & - & - & - & - & - & - & - & - & - & - & - & - & - & - \\
\hline 43 & - & - & - & - & - & - & - & $<$ & - & - & - & - & - & - & - \\
\hline 44 & - & - & - & - & - & - & - & $<$ & - & - & - & - & - & - & - \\
\hline 45 & - & - & - & - & - & - & - & - & - & - & - & - & - & - & - \\
\hline 46 & - & - & $<$ & - & $<$ & - & - & $<$ & - & - & - & - & $<$ & - & $<$ \\
\hline 47 & - & - & - & - & - & - & - & - & - & - & - & - & - & - & - \\
\hline 48 & - & - & - & - & - & - & - & - & - & - & - & - & - & - & - \\
\hline 49 & - & - & - & - & - & - & - & - & - & - & - & - & - & - & - \\
\hline 50 & - & - & - & - & $<$ & - & - & - & - & - & - & - & - & - & $<$ \\
\hline 51 & - & - & $<$ & - & $<$ & - & - & $<$ & - & - & - & - & $<$ & - & $<$ \\
\hline 52 & - & - & - & - & - & - & - & - & - & - & - & - & - & - & - \\
\hline 53 & - & - & - & - & $<$ & - & - & - & - & - & - & - & - & - & $<$ \\
\hline
\end{tabular}

(Continued on next page) 
Table 9 (Continued from previous page)

\begin{tabular}{|c|c|c|c|c|c|c|c|c|c|c|c|c|c|c|c|}
\hline & 108 & 109 & 110 & 111 & 112 & 113 & 114 & 115 & 116 & 117 & 118 & 119 & 120 & 121 & 122 \\
\hline 54 & - & - & $<$ & - & $<$ & - & - & $<$ & - & - & - & - & $<$ & - & $<$ \\
\hline 55 & - & - & - & - & - & - & - & - & - & - & - & - & - & - & - \\
\hline 56 & - & - & $<$ & - & $<$ & - & - & $<$ & - & - & - & - & $<$ & - & $<$ \\
\hline 57 & - & - & - & - & - & - & - & - & - & - & - & - & - & - & - \\
\hline 58 & - & - & $<$ & $<$ & $<$ & - & - & $<$ & $<$ & - & - & - & $<$ & $<$ & $<$ \\
\hline 59 & - & - & $<$ & - & $<$ & - & - & $<$ & - & - & - & - & $<$ & - & $<$ \\
\hline 60 & - & - & - & - & - & - & - & - & - & - & - & - & - & - & - \\
\hline 61 & - & - & $<$ & - & $<$ & - & - & $<$ & - & - & - & - & - & - & - \\
\hline 62 & - & - & - & - & - & - & - & - & - & - & - & - & - & - & - \\
\hline 63 & - & - & - & - & - & - & - & - & - & - & - & - & - & - & - \\
\hline 64 & - & - & - & - & $<$ & - & - & - & - & - & - & - & - & - & - \\
\hline 65 & - & - & - & - & - & - & - & - & - & - & - & - & - & - & - \\
\hline 66 & - & - & - & - & - & - & - & - & - & - & - & - & - & - & - \\
\hline 67 & - & - & $<$ & - & $<$ & - & - & $<$ & - & - & - & - & - & - & - \\
\hline 68 & - & - & - & - & - & - & - & - & - & - & - & - & - & - & - \\
\hline 69 & - & - & - & - & - & - & - & - & - & - & - & - & - & - & - \\
\hline 70 & - & - & - & - & - & - & - & $<$ & $<$ & - & - & - & - & - & - \\
\hline 71 & - & - & - & - & - & - & - & - & - & - & - & - & - & - & - \\
\hline 72 & - & - & $<$ & - & $<$ & - & - & $<$ & - & - & - & - & - & - & - \\
\hline 73 & - & - & $<$ & $<$ & $<$ & - & - & $<$ & $<$ & - & - & - & - & - & - \\
\hline 74 & - & - & - & - & - & - & - & - & - & - & - & - & - & - & - \\
\hline 75 & - & - & - & - & - & - & - & - & - & - & - & - & - & - & - \\
\hline 76 & - & - & - & - & - & - & - & $<$ & - & - & - & - & - & - & - \\
\hline 77 & - & - & - & - & - & - & - & - & - & - & - & - & - & - & - \\
\hline 78 & - & - & $<$ & $<$ & $<$ & - & - & $<$ & $<$ & - & - & - & - & - & - \\
\hline 79 & - & - & - & - & - & - & - & - & - & - & - & - & - & - & - \\
\hline 80 & - & - & $<$ & $<$ & $<$ & - & - & $<$ & $<$ & - & - & - & - & - & - \\
\hline 81 & - & - & - & - & - & - & - & - & - & - & - & - & - & - & - \\
\hline 82 & - & - & - & - & - & - & - & $<$ & - & - & - & - & - & - & - \\
\hline 83 & - & - & - & - & - & - & - & - & - & - & - & - & - & - & - \\
\hline 84 & - & - & - & - & - & - & - & - & - & - & - & - & - & - & - \\
\hline 85 & - & - & - & - & - & - & - & - & - & - & - & - & - & - & - \\
\hline 86 & - & - & - & - & - & - & - & $<$ & - & - & - & - & - & - & - \\
\hline 87 & - & - & - & - & - & - & - & $<$ & $<$ & - & - & - & - & - & - \\
\hline 88 & - & - & - & - & - & - & - & - & - & - & - & - & - & - & - \\
\hline 89 & - & - & - & - & - & - & - & $<$ & $<$ & - & - & - & - & - & - \\
\hline 90 & - & - & - & - & - & - & - & - & - & - & - & - & - & - & - \\
\hline
\end{tabular}

(Continued on next page) 
Table 9 (Continued from previous page)

\begin{tabular}{|c|c|c|c|c|c|c|c|c|c|c|c|c|c|c|c|}
\hline & 108 & 109 & 110 & 111 & 112 & 113 & 114 & 115 & 116 & 117 & 118 & 119 & 120 & 121 & 122 \\
\hline 91 & - & - & $<$ & - & $<$ & - & - & $<$ & - & - & - & - & $<$ & - & $<$ \\
\hline 92 & - & - & - & - & - & - & - & - & - & - & - & - & - & - & - \\
\hline 93 & - & - & - & - & - & - & - & - & - & - & - & - & - & - & - \\
\hline 94 & - & - & - & - & $<$ & - & - & - & - & - & - & - & - & - & $<$ \\
\hline 95 & - & - & $<$ & - & $<$ & - & - & $<$ & - & - & - & - & $<$ & - & $<$ \\
\hline 96 & - & - & $<$ & $<$ & $<$ & - & - & $<$ & $<$ & - & - & - & $<$ & $<$ & $<$ \\
\hline 97 & - & - & - & - & - & - & - & - & - & - & - & - & - & - & - \\
\hline 98 & - & - & $<$ & $<$ & $<$ & - & - & $<$ & $<$ & - & - & - & $<$ & $<$ & $<$ \\
\hline 99 & - & - & - & - & - & - & - & - & - & - & - & - & - & - & - \\
\hline 100 & - & - & - & - & - & - & - & - & - & - & - & - & - & - & - \\
\hline 101 & - & - & - & - & - & - & - & - & - & - & - & - & - & - & - \\
\hline 102 & - & - & - & - & - & - & - & - & - & - & - & - & - & - & - \\
\hline 103 & - & - & - & - & - & - & - & - & - & - & - & - & - & - & - \\
\hline 104 & - & - & - & - & - & - & - & - & - & - & - & - & - & - & - \\
\hline 105 & - & - & - & - & - & - & - & - & - & - & - & - & - & - & - \\
\hline 106 & - & - & - & - & - & - & - & - & - & - & - & - & - & - & - \\
\hline 107 & - & - & - & - & - & - & - & - & - & - & - & - & - & - & - \\
\hline 108 & $=$ & - & - & - & - & - & - & - & - & - & - & - & - & - & - \\
\hline 109 & - & $=$ & $<$ & - & $<$ & - & - & - & - & - & - & - & - & - & - \\
\hline 110 & - & $>$ & $=$ & - & $<$ & - & - & - & - & - & - & $>$ & $>$ & - & - \\
\hline 111 & - & - & - & $=$ & - & - & - & - & - & - & - & - & - & - & - \\
\hline 112 & - & $>$ & $>$ & - & $=$ & - & - & - & - & - & - & $>$ & $>$ & - & $>$ \\
\hline 113 & - & - & - & - & - & $=$ & - & - & - & - & - & - & - & - & - \\
\hline 114 & - & - & - & - & - & - & $=$ & $<$ & - & - & - & - & - & - & - \\
\hline 115 & - & - & - & - & - & - & $>$ & $=$ & - & - & - & - & - & - & - \\
\hline 116 & - & - & - & - & - & - & - & - & $=$ & - & - & - & - & - & - \\
\hline 117 & - & - & - & - & - & - & - & - & - & $=$ & - & - & - & - & - \\
\hline 118 & - & - & - & - & - & - & - & - & - & - & $=$ & - & - & - & - \\
\hline 119 & - & - & $<$ & - & $<$ & - & - & - & - & - & - & $=$ & $<$ & - & $<$ \\
\hline 120 & - & - & $<$ & - & $<$ & - & - & - & - & - & - & $>$ & $=$ & - & $<$ \\
\hline 121 & - & - & - & - & - & - & - & - & - & - & - & - & - & $=$ & - \\
\hline 122 & - & - & - & - & $<$ & - & - & - & - & - & - & $>$ & $>$ & - & $=$ \\
\hline 123 & - & - & - & - & - & - & - & - & - & - & - & - & - & - & - \\
\hline 124 & - & - & - & - & - & - & - & - & - & - & - & - & - & - & - \\
\hline 125 & - & - & - & - & - & - & - & - & - & - & - & - & - & - & - \\
\hline 126 & - & - & - & - & - & - & - & - & - & - & - & - & - & - & - \\
\hline 127 & - & - & - & - & - & - & - & - & - & - & - & - & - & - & - \\
\hline
\end{tabular}

(Continued on next page) 
Table 9 (Continued from previous page)

\begin{tabular}{|c|c|c|c|c|c|c|c|c|c|c|c|c|c|c|c|}
\hline & 108 & 109 & 110 & 111 & 112 & 113 & 114 & 115 & 116 & 117 & 118 & 119 & 120 & 121 & 122 \\
\hline 128 & - & - & - & - & - & - & - & - & - & - & - & - & - & - & - \\
\hline 129 & - & - & - & - & - & - & - & - & - & - & - & - & - & - & - \\
\hline 130 & - & - & - & - & - & - & - & - & - & - & - & - & - & - & - \\
\hline 131 & - & - & - & - & - & - & - & - & - & - & - & - & - & - & - \\
\hline 132 & - & - & - & - & - & - & - & - & - & - & - & - & - & - & - \\
\hline 133 & - & - & - & - & - & - & - & - & - & - & - & - & - & - & - \\
\hline 134 & - & - & - & - & - & - & - & - & - & - & - & - & - & - & - \\
\hline 135 & - & - & - & - & - & - & - & - & - & - & - & - & - & - & - \\
\hline 136 & - & - & - & - & - & - & - & - & - & - & - & - & - & - & - \\
\hline 137 & - & - & - & - & - & - & - & - & - & - & - & - & - & - & - \\
\hline 138 & - & - & - & - & - & - & - & - & - & - & - & - & - & - & - \\
\hline 139 & - & - & $<$ & - & $<$ & - & - & $<$ & - & - & - & - & - & - & - \\
\hline 140 & - & - & $<$ & - & $<$ & - & - & $<$ & - & - & - & - & - & - & - \\
\hline 141 & - & - & $<$ & - & $<$ & - & - & $<$ & - & - & - & - & - & - & - \\
\hline 142 & - & - & - & - & - & - & - & $<$ & - & - & - & - & - & - & - \\
\hline 143 & - & - & - & - & - & - & - & $<$ & - & - & - & - & - & - & - \\
\hline 144 & - & - & - & - & - & - & - & $<$ & - & - & - & - & - & - & - \\
\hline 145 & - & - & $<$ & - & $<$ & - & - & $<$ & - & - & - & - & $<$ & - & $<$ \\
\hline 146 & - & - & - & - & - & - & - & $<$ & - & - & - & - & - & - & - \\
\hline 147 & - & - & - & - & - & - & - & $<$ & - & - & - & - & - & - & - \\
\hline 148 & - & - & - & - & - & - & - & $<$ & - & - & - & - & - & - & - \\
\hline 149 & - & - & $<$ & - & $<$ & - & - & $<$ & - & - & - & - & $<$ & - & $<$ \\
\hline 166 & - & - & $<$ & - & $<$ & - & - & $<$ & - & - & - & - & - & - & - \\
\hline 167 & - & - & - & - & - & - & - & $<$ & - & - & - & - & - & - & - \\
\hline 168 & - & - & - & - & - & - & - & $<$ & - & - & - & - & - & - & - \\
\hline 169 & - & - & $<$ & - & $<$ & - & - & $<$ & - & - & - & - & $<$ & - & $<$ \\
\hline 170 & - & - & - & - & - & - & - & $<$ & - & - & - & - & - & - & - \\
\hline 171 & - & - & - & - & - & - & - & - & - & - & - & - & - & - & - \\
\hline 172 & - & - & - & - & - & - & - & - & - & - & - & - & - & - & - \\
\hline 173 & - & - & - & - & - & - & - & - & - & - & - & - & - & - & - \\
\hline 174 & - & - & - & - & - & - & - & - & - & - & - & - & - & - & - \\
\hline 175 & - & - & - & - & - & - & - & - & - & - & - & - & - & - & - \\
\hline 176 & - & - & - & - & - & - & - & - & - & - & - & - & - & - & - \\
\hline 177 & - & - & - & - & - & - & - & - & - & - & - & - & - & - & - \\
\hline 178 & - & - & - & - & - & - & - & - & - & - & - & - & - & - & - \\
\hline 179 & - & - & - & - & - & - & - & - & - & - & - & - & - & - & - \\
\hline 180 & - & - & - & - & - & - & - & - & - & - & - & - & - & - & - \\
\hline
\end{tabular}

(Continued on next page) 
Table 9 (Continued from previous page)

\begin{tabular}{|c|ccccccccccccccc|}
\hline & 108 & 109 & 110 & 111 & 112 & 113 & 114 & 115 & 116 & 117 & 118 & 119 & 120 & 121 & 122 \\
\hline 181 & - & - & - & - & - & - & - & $<$ & - & - & - & - & - & - & - \\
\hline 182 & - & - & - & - & - & - & - & $<$ & - & - & - & - & - & - & - \\
\hline 183 & - & - & - & - & - & - & - & $<$ & - & - & - & - & - & - & - \\
\hline 184 & - & - & - & - & - & - & - & $<$ & - & - & - & - & - & - & - \\
\hline 185 & - & - & $<$ & - & $<$ & - & - & $<$ & - & - & - & - & $<$ & - & $<$ \\
\hline 186 & - & - & - & - & - & - & - & - & - & - & - & - & - & - & - \\
\hline 187 & - & - & - & - & - & - & - & - & - & - & - & - & - & - & - \\
\hline 188 & - & - & - & - & - & - & - & - & - & - & - & - & - & - & - \\
\hline 189 & - & - & - & - & - & - & - & - & - & - & - & - & - & - & - \\
\hline 190 & - & - & - & - & - & - & - & - & - & - & - & - & - & - & - \\
\hline 191 & - & - & - & - & - & - & - & - & - & - & - & - & - & - & - \\
\hline 192 & - & - & $<$ & - & $<$ & - & - & $<$ & - & - & - & - & - & - & - \\
\hline 193 & - & - & $<$ & - & $<$ & - & - & $<$ & - & - & - & - & $<$ & - & $<$ \\
\hline 194 & - & - & - & - & - & - & - & $<$ & - & - & - & - & - & - & - \\
\hline 195 & - & - & - & - & - & - & - & $<$ & - & - & - & - & - & - & - \\
\hline 196 & - & - & - & - & - & - & - & $<$ & - & - & - & - & - & - & - \\
\hline 197 & - & - & $<$ & - & $<$ & - & - & $<$ & - & - & - & - & $<$ & - & $<$ \\
\hline 198 & - & - & - & - & - & - & - & $<$ & - & - & - & - & - & - & - \\
\hline
\end{tabular}

Table 10. Relations between the intuitionistic fuzzy implications $\rightarrow_{123}, \ldots, \rightarrow_{137}$

\begin{tabular}{|c|ccccccccccccccc|}
\hline & 123 & 124 & 125 & 126 & 127 & 128 & 129 & 130 & 131 & 132 & 133 & 134 & 135 & 136 & 137 \\
\hline 1 & - & - & $<$ & - & $<$ & - & - & $<$ & - & - & - & - & - & - & - \\
\hline 2 & - & - & - & - & - & - & - & - & - & - & - & - & - & - & - \\
\hline 3 & - & - & - & - & - & - & - & - & - & - & - & - & - & - & - \\
\hline 4 & - & - & $<$ & - & $<$ & - & - & $<$ & - & - & - & - & - & - & - \\
\hline 5 & - & - & - & - & - & - & - & - & - & - & - & - & - & - & - \\
\hline 6 & - & - & - & - & $<$ & - & - & - & - & - & - & - & - & - & - \\
\hline 7 & - & - & $<$ & - & $<$ & - & - & $<$ & - & - & - & - & - & - & - \\
\hline 8 & - & - & - & - & - & - & - & - & - & - & - & - & - & - & - \\
\hline 9 & - & - & - & - & - & - & - & - & - & - & - & - & - & - & - \\
\hline 10 & - & - & $<$ & - & $<$ & - & - & $<$ & - & - & - & - & - & - & - \\
\hline 11 & - & - & - & - & - & - & - & - & - & - & - & - & - & - & - \\
\hline 12 & - & - & $<$ & - & $<$ & - & - & $<$ & - & - & - & - & $<$ & - & $<$ \\
\hline 13 & - & - & - & - & $<$ & - & - & - & - & - & - & - & - & - & - \\
\hline 14 & - & - & - & - & - & - & - & - & - & - & - & - & - & - & - \\
\hline 15 & - & - & - & - & - & - & - & - & - & - & - & - & - & - & - \\
\hline 16 & - & - & - & - & - & - & - & $<$ & $<$ & - & - & - & - & - & - \\
\hline
\end{tabular}

(Continued on next page) 
Table 10 (Continued from previous page)

\begin{tabular}{|c|c|c|c|c|c|c|c|c|c|c|c|c|c|c|c|}
\hline & 123 & 124 & 125 & 126 & 127 & 128 & 129 & 130 & 131 & 132 & 133 & 134 & 135 & 136 & 137 \\
\hline 17 & - & - & - & - & - & - & - & - & - & - & - & - & - & - & - \\
\hline 18 & - & - & $<$ & - & $<$ & - & - & $<$ & - & - & - & - & - & - & - \\
\hline 19 & - & - & $<$ & $<$ & $<$ & - & - & $<$ & $<$ & - & - & - & - & - & - \\
\hline 20 & - & - & - & - & - & - & - & - & - & - & - & - & - & - & - \\
\hline 21 & - & - & - & - & - & - & - & - & - & - & - & - & - & - & - \\
\hline 22 & - & - & - & - & - & - & - & $<$ & - & - & - & - & - & - & - \\
\hline 23 & - & - & - & - & - & - & - & - & - & - & - & - & - & - & - \\
\hline 24 & - & - & - & - & - & - & - & - & - & - & - & - & - & - & - \\
\hline 25 & - & - & $<$ & $<$ & $<$ & - & - & $<$ & $<$ & - & - & - & - & - & - \\
\hline 26 & - & - & $<$ & $<$ & $<$ & - & - & $<$ & $<$ & - & - & - & - & - & - \\
\hline 27 & - & - & - & - & - & - & - & - & - & - & - & - & - & - & - \\
\hline 28 & - & - & $<$ & $<$ & $<$ & - & - & $<$ & $<$ & - & - & - & - & - & - \\
\hline 29 & - & - & - & - & - & - & - & - & - & - & - & - & - & - & - \\
\hline 30 & - & - & - & - & - & - & - & $<$ & - & - & - & - & - & - & - \\
\hline 31 & - & - & - & - & - & - & - & - & - & - & - & - & - & - & - \\
\hline 32 & - & - & - & - & - & - & - & - & - & - & - & - & - & - & - \\
\hline 33 & - & - & - & - & - & - & - & $<$ & - & - & - & - & - & - & - \\
\hline 34 & - & - & - & - & - & - & - & - & - & - & - & - & - & - & - \\
\hline 35 & - & - & - & - & - & - & - & - & - & $<$ & - & - & - & - & - \\
\hline 36 & - & - & - & - & - & - & - & $<$ & - & - & - & - & - & - & - \\
\hline 37 & - & - & - & - & - & - & - & - & - & - & - & - & - & - & - \\
\hline 38 & - & - & - & - & - & - & - & - & - & - & - & - & - & - & - \\
\hline 39 & - & - & - & - & - & - & - & $<$ & - & - & - & - & - & - & - \\
\hline 40 & - & - & - & - & - & - & - & - & - & - & - & - & - & - & - \\
\hline 41 & - & - & - & - & - & - & - & $<$ & $<$ & - & - & - & - & - & - \\
\hline 42 & - & - & - & - & - & - & - & - & - & - & - & - & - & - & - \\
\hline 43 & - & - & - & - & - & - & - & $<$ & $<$ & - & - & - & - & - & - \\
\hline 44 & - & - & - & - & - & - & - & $<$ & $<$ & - & - & - & - & - & - \\
\hline 45 & - & - & - & - & - & - & - & - & - & - & - & - & - & - & - \\
\hline 46 & - & - & $<$ & - & $<$ & - & - & $<$ & - & - & - & - & $<$ & - & $<$ \\
\hline 47 & - & - & - & - & - & - & - & - & - & - & - & - & - & - & - \\
\hline 48 & - & - & - & - & - & - & - & - & - & - & - & - & - & - & - \\
\hline 49 & - & - & - & - & - & - & - & - & - & - & - & - & - & - & - \\
\hline 50 & - & - & - & - & $<$ & - & - & - & - & - & - & - & - & - & $<$ \\
\hline 51 & - & - & $<$ & - & $<$ & - & - & $<$ & - & - & - & - & $<$ & - & $<$ \\
\hline 52 & - & - & - & - & - & - & - & - & - & - & - & - & - & - & - \\
\hline 53 & - & - & - & - & $<$ & - & - & - & - & - & - & - & - & - & $<$ \\
\hline
\end{tabular}

(Continued on next page) 
Table 10 (Continued from previous page)

\begin{tabular}{|c|c|c|c|c|c|c|c|c|c|c|c|c|c|c|c|}
\hline & 123 & 124 & 125 & 126 & 127 & 128 & 129 & 130 & 131 & 132 & 133 & 134 & 135 & 136 & 137 \\
\hline 54 & - & - & $<$ & - & $<$ & - & - & $<$ & - & - & - & - & $<$ & - & $<$ \\
\hline 55 & - & - & - & - & - & - & - & - & - & - & - & - & - & - & - \\
\hline 56 & - & - & $<$ & $<$ & $<$ & - & - & $<$ & $<$ & - & - & - & $<$ & $<$ & $<$ \\
\hline 57 & - & - & - & - & - & - & - & - & - & - & - & - & - & - & - \\
\hline 58 & - & - & $<$ & $<$ & $<$ & - & - & $<$ & $<$ & - & - & - & $<$ & $<$ & $<$ \\
\hline 59 & - & - & $<$ & $<$ & $<$ & - & - & $<$ & $<$ & - & - & - & $<$ & $<$ & $<$ \\
\hline 60 & - & - & - & - & - & - & - & - & - & - & - & - & - & - & - \\
\hline 61 & - & - & $<$ & - & $<$ & - & - & $<$ & - & - & - & - & - & - & - \\
\hline 62 & - & - & - & - & - & - & - & - & - & - & - & - & - & - & - \\
\hline 63 & - & - & - & - & - & - & - & - & - & - & - & - & - & - & - \\
\hline 64 & - & - & - & - & $<$ & - & - & - & - & - & - & - & - & - & - \\
\hline 65 & - & - & - & - & - & - & - & - & - & - & - & - & - & - & - \\
\hline 66 & - & - & - & - & $<$ & - & - & - & - & - & - & - & - & - & - \\
\hline 67 & - & - & $<$ & - & $<$ & - & - & $<$ & - & - & - & - & - & - & - \\
\hline 68 & - & - & - & - & - & - & - & - & - & - & - & - & - & - & - \\
\hline 69 & - & - & - & - & - & - & - & - & - & - & - & - & - & - & - \\
\hline 70 & - & - & - & - & - & - & - & $<$ & - & - & - & - & - & - & - \\
\hline 71 & - & - & $<$ & - & $<$ & - & - & - & - & - & - & - & - & - & - \\
\hline 72 & - & - & $<$ & - & $<$ & - & - & $<$ & - & - & - & - & - & - & - \\
\hline 73 & - & - & $<$ & - & $<$ & - & - & $<$ & - & - & - & - & - & - & - \\
\hline 74 & - & - & - & - & - & - & - & - & - & - & - & - & - & - & - \\
\hline 75 & - & - & - & - & - & - & - & - & - & - & - & - & - & - & - \\
\hline 76 & - & - & - & - & - & - & - & $<$ & - & - & - & - & - & - & - \\
\hline 77 & - & - & - & - & - & - & - & - & - & - & - & - & - & - & - \\
\hline 78 & - & - & $<$ & - & $<$ & - & - & $<$ & - & - & - & - & - & - & - \\
\hline 79 & - & - & - & - & - & - & - & - & - & - & - & - & - & - & - \\
\hline 80 & - & - & $<$ & - & $<$ & - & - & $<$ & - & - & - & - & - & - & - \\
\hline 81 & - & - & - & - & - & - & - & - & - & - & - & - & - & - & - \\
\hline 82 & - & - & - & - & - & - & - & $<$ & - & - & - & - & - & - & - \\
\hline 83 & - & - & - & - & - & - & - & - & - & - & - & - & - & - & - \\
\hline 84 & - & - & - & - & - & - & - & - & - & - & - & - & - & - & - \\
\hline 85 & - & - & - & - & - & - & - & - & - & - & - & - & - & - & - \\
\hline 86 & - & - & - & - & - & - & - & $<$ & - & - & - & - & - & - & - \\
\hline 87 & - & - & - & - & - & - & - & $<$ & - & - & - & - & - & - & - \\
\hline 88 & - & - & - & - & - & - & - & - & - & - & - & - & - & - & - \\
\hline 89 & - & - & - & - & - & - & - & $<$ & - & - & - & - & - & - & - \\
\hline 90 & - & - & - & - & - & - & - & - & - & - & - & - & - & - & - \\
\hline
\end{tabular}

(Continued on next page) 
Table 10 (Continued from previous page)

\begin{tabular}{|c|c|c|c|c|c|c|c|c|c|c|c|c|c|c|c|}
\hline & 123 & 124 & 125 & 126 & 127 & 128 & 129 & 130 & 131 & 132 & 133 & 134 & 135 & 136 & 137 \\
\hline 91 & - & - & $<$ & - & $<$ & - & - & $<$ & - & - & - & - & $<$ & - & $<$ \\
\hline 92 & - & - & - & - & - & - & - & - & - & - & - & - & - & - & - \\
\hline 93 & - & - & - & - & - & - & - & - & - & - & - & - & - & - & - \\
\hline 94 & - & - & - & - & $<$ & - & - & - & - & - & - & - & - & - & $<$ \\
\hline 95 & - & - & $<$ & - & $<$ & - & - & $<$ & - & - & - & - & $<$ & - & $<$ \\
\hline 96 & - & - & $<$ & - & $<$ & - & - & $<$ & - & - & - & - & $<$ & - & $<$ \\
\hline 97 & - & - & - & - & - & - & - & - & - & - & - & - & - & - & - \\
\hline 98 & - & - & $<$ & - & $<$ & - & - & $<$ & - & - & - & - & $<$ & - & $<$ \\
\hline 99 & - & - & - & - & - & - & - & - & - & - & - & - & - & - & - \\
\hline 100 & - & - & - & - & - & - & - & - & - & - & - & - & - & - & - \\
\hline 101 & - & - & - & - & - & - & - & - & - & - & - & - & - & - & - \\
\hline 102 & - & - & - & - & - & - & - & - & - & - & - & - & - & - & - \\
\hline 103 & - & - & - & - & - & - & - & - & - & - & - & - & - & - & - \\
\hline 104 & - & - & - & - & - & - & - & - & - & - & - & - & - & - & - \\
\hline 105 & - & - & - & - & - & - & - & - & - & - & - & - & - & - & - \\
\hline 106 & - & - & - & - & - & - & - & - & - & - & - & - & - & - & - \\
\hline 107 & - & - & - & - & - & - & - & - & - & - & - & - & - & - & - \\
\hline 108 & - & - & - & - & - & - & - & - & - & - & - & - & - & - & - \\
\hline 109 & - & - & - & - & - & - & - & - & - & - & - & - & - & - & - \\
\hline 110 & - & - & - & - & - & - & - & - & - & - & - & - & - & - & - \\
\hline 111 & - & - & - & - & - & - & - & - & - & - & - & - & - & - & - \\
\hline 112 & - & - & - & - & - & - & - & - & - & - & - & - & - & - & - \\
\hline 113 & - & - & - & - & - & - & - & - & - & - & - & - & - & - & - \\
\hline 114 & - & - & - & - & - & - & - & - & - & - & - & - & - & - & - \\
\hline 115 & - & - & - & - & - & - & - & - & - & - & - & - & - & - & - \\
\hline 116 & - & - & - & - & - & - & - & - & - & - & - & - & - & - & - \\
\hline 117 & - & - & - & - & - & - & - & - & - & - & - & - & - & - & - \\
\hline 118 & - & - & - & - & - & - & - & - & - & - & - & - & - & - & - \\
\hline 119 & - & - & - & - & - & - & - & - & - & - & - & - & - & - & - \\
\hline 120 & - & - & - & - & - & - & - & - & - & - & - & - & - & - & - \\
\hline 121 & - & - & - & - & - & - & - & - & - & - & - & - & - & - & - \\
\hline 122 & - & - & - & - & - & - & - & - & - & - & - & - & - & - & - \\
\hline 123 & $=$ & - & - & - & - & - & - & - & - & - & - & - & - & - & - \\
\hline 124 & - & $=$ & $<$ & - & $<$ & - & - & - & - & - & - & - & - & - & - \\
\hline 125 & - & $>$ & $=$ & - & $<$ & - & - & - & - & - & - & $>$ & $>$ & - & - \\
\hline 126 & - & - & - & $=$ & - & - & - & - & - & - & - & - & - & - & - \\
\hline 127 & - & $>$ & $>$ & - & $=$ & - & - & - & - & - & - & $>$ & $>$ & - & $>$ \\
\hline \multicolumn{16}{|c|}{ (Continued on next page) } \\
\hline
\end{tabular}


Table 10 (Continued from previous page)

\begin{tabular}{|c|c|c|c|c|c|c|c|c|c|c|c|c|c|c|c|}
\hline & 123 & 124 & 125 & 126 & 127 & 128 & 129 & 130 & 131 & 132 & 133 & 134 & 135 & 136 & 137 \\
\hline 128 & - & - & - & - & - & $=$ & - & - & - & - & - & - & - & - & - \\
\hline 129 & - & - & - & - & - & - & $=$ & $<$ & - & - & - & - & - & - & - \\
\hline 130 & - & - & - & - & - & - & $>$ & $=$ & - & - & - & - & - & - & - \\
\hline 131 & - & - & - & - & - & - & - & - & $=$ & - & - & - & - & - & - \\
\hline 132 & - & - & - & - & - & - & - & - & - & $=$ & - & - & - & - & - \\
\hline 133 & - & - & - & - & - & - & - & - & - & - & $=$ & - & - & - & - \\
\hline 134 & - & - & $<$ & - & $<$ & - & - & - & - & - & - & $=$ & $<$ & - & $<$ \\
\hline 135 & - & - & $<$ & - & $<$ & - & - & - & - & - & - & $>$ & $=$ & - & $<$ \\
\hline 136 & - & - & - & - & - & - & - & - & - & - & - & - & - & $=$ & - \\
\hline 137 & - & - & - & - & $<$ & - & - & - & - & - & - & $>$ & $>$ & - & $=$ \\
\hline 138 & - & - & - & - & - & - & - & - & - & - & - & - & - & - & - \\
\hline 139 & - & - & $<$ & - & $<$ & - & - & $<$ & - & - & - & - & - & - & - \\
\hline 140 & - & - & $<$ & - & $<$ & - & - & $<$ & - & - & - & - & - & - & - \\
\hline 141 & - & - & $<$ & - & $<$ & - & - & $<$ & - & - & - & - & - & - & - \\
\hline 142 & - & - & - & - & - & - & - & $<$ & - & - & - & - & - & - & - \\
\hline 143 & - & - & - & - & - & - & - & $<$ & - & - & - & - & - & - & - \\
\hline 144 & - & - & - & - & - & - & - & $<$ & - & - & - & - & - & - & - \\
\hline 145 & - & - & $<$ & - & $<$ & - & - & $<$ & - & - & - & - & $<$ & - & $<$ \\
\hline 146 & - & - & - & - & - & - & - & $<$ & - & - & - & - & - & - & - \\
\hline 147 & - & - & - & - & - & - & - & $<$ & - & - & - & - & - & - & - \\
\hline 148 & - & - & - & - & - & - & - & $<$ & - & - & - & - & - & - & - \\
\hline 149 & - & - & $<$ & - & $<$ & - & - & $<$ & - & - & - & - & $<$ & - & $<$ \\
\hline 166 & - & - & $<$ & - & $<$ & - & - & $<$ & - & - & - & - & - & - & - \\
\hline 167 & - & - & - & - & - & - & - & $<$ & - & - & - & - & - & - & - \\
\hline 168 & - & - & - & - & - & - & - & $<$ & - & - & - & - & - & - & - \\
\hline 169 & - & - & $<$ & - & $<$ & - & - & $<$ & - & - & - & - & $<$ & - & $<$ \\
\hline 170 & - & - & - & - & - & - & - & $<$ & - & - & - & - & - & - & - \\
\hline 171 & - & - & - & - & - & - & - & - & - & - & - & - & - & - & - \\
\hline 172 & - & - & - & - & - & - & - & - & - & - & - & - & - & - & - \\
\hline 173 & - & - & - & - & - & - & - & - & - & - & - & - & - & - & - \\
\hline 174 & - & - & - & - & - & - & - & - & - & - & - & - & - & - & - \\
\hline 175 & - & - & - & - & - & - & - & - & - & - & - & - & - & - & - \\
\hline 176 & - & - & - & - & - & - & - & - & - & - & - & - & - & - & - \\
\hline 177 & - & - & - & - & - & - & - & - & - & - & - & - & - & - & - \\
\hline 178 & - & - & - & - & - & - & - & - & - & - & - & - & - & - & - \\
\hline 179 & - & - & - & - & - & - & - & - & - & - & - & - & - & - & - \\
\hline 180 & - & - & - & - & - & - & - & - & - & - & - & - & - & - & - \\
\hline
\end{tabular}

(Continued on next page) 
Table 10 (Continued from previous page)

\begin{tabular}{|c|ccccccccccccccc|}
\hline & 123 & 124 & 125 & 126 & 127 & 128 & 129 & 130 & 131 & 132 & 133 & 134 & 135 & 136 & 137 \\
\hline 181 & - & - & - & - & - & - & - & $<$ & $<$ & - & - & - & - & - & - \\
\hline 182 & - & - & - & - & - & - & - & $<$ & $<$ & - & - & - & - & - & - \\
\hline 183 & - & - & - & - & - & - & - & $<$ & $<$ & - & - & - & - & - & - \\
\hline 184 & - & - & - & - & - & - & - & $<$ & $<$ & - & - & - & - & - & - \\
\hline 185 & - & - & $<$ & $<$ & $<$ & - & - & $<$ & $<$ & - & - & - & $<$ & $<$ & $<$ \\
\hline 186 & - & - & - & - & - & - & - & - & - & - & - & - & - & - & - \\
\hline 187 & - & - & - & - & - & - & - & - & - & - & - & - & - & - & - \\
\hline 188 & - & - & - & - & - & - & - & - & - & - & - & - & - & - & - \\
\hline 189 & - & - & - & - & - & - & - & - & - & - & - & - & - & - & - \\
\hline 190 & - & - & - & - & - & - & - & - & - & - & - & - & - & - & - \\
\hline 191 & - & - & - & - & - & - & - & - & - & - & - & - & - & - & - \\
\hline 192 & - & - & $<$ & - & $<$ & - & - & $<$ & - & - & - & - & - & - & - \\
\hline 193 & - & - & $<$ & - & $<$ & - & - & $<$ & - & - & - & - & $<$ & - & $<$ \\
\hline 194 & - & - & - & - & - & - & - & $<$ & - & - & - & - & - & - & - \\
\hline 195 & - & - & - & - & - & - & - & $<$ & - & - & - & - & - & - & - \\
\hline 196 & - & - & - & - & - & - & - & $<$ & - & - & - & - & - & - & - \\
\hline 197 & - & - & $<$ & - & $<$ & - & - & $<$ & - & - & - & - & $<$ & - & $<$ \\
\hline 198 & - & - & - & - & - & - & - & $<$ & - & - & - & - & - & - & - \\
\hline
\end{tabular}

Table 11. Relations between the intuitionistic fuzzy implications $\rightarrow_{138}, \ldots, \rightarrow_{149}$ and $\rightarrow_{166}, \ldots, \rightarrow_{168}$

\begin{tabular}{|c|ccccccccccccccc|}
\hline & 138 & 139 & 140 & 141 & 142 & 143 & 144 & 145 & 146 & 147 & 148 & 149 & 166 & 167 & 168 \\
\hline 1 & - & - & - & - & - & - & - & - & - & - & - & - & $>$ & - & - \\
\hline 2 & - & - & - & - & - & - & - & - & - & - & - & - & - & - & - \\
\hline 3 & - & - & - & - & - & - & - & - & - & - & - & - & - & - & - \\
\hline 4 & - & $>$ & $>$ & $>$ & - & - & - & $>$ & - & - & - & $>$ & $>$ & - & - \\
\hline 5 & - & $>$ & $>$ & $>$ & - & - & - & $>$ & - & - & - & $>$ & $>$ & - & - \\
\hline 6 & - & - & - & - & - & - & - & - & - & - & - & - & - & - & - \\
\hline 7 & - & - & - & - & - & - & - & - & - & - & - & - & $<$ & - & - \\
\hline 8 & - & - & - & - & - & - & - & - & - & - & - & - & - & - & - \\
\hline 9 & - & - & - & - & - & - & - & - & - & - & - & - & - & - & - \\
\hline 10 & - & - & - & - & - & - & - & - & - & - & - & - & - & - & - \\
\hline 11 & - & - & - & - & - & - & - & - & - & - & - & - & - & - & - \\
\hline 12 & - & - & - & - & - & - & - & $>$ & - & - & - & $>$ & - & - & - \\
\hline 13 & - & $>$ & $>$ & $>$ & - & - & - & $>$ & - & - & - & $>$ & $>$ & - & - \\
\hline 14 & - & - & - & - & - & - & - & - & - & - & - & - & - & - & - \\
\hline 15 & - & - & - & - & - & - & - & - & - & - & - & - & - & - & - \\
\hline
\end{tabular}

(Continued on next page) 
Table 11 (Continued from previous page)

\begin{tabular}{|c|c|c|c|c|c|c|c|c|c|c|c|c|c|c|c|}
\hline & 138 & 139 & 140 & 141 & 142 & 143 & 144 & 145 & 146 & 147 & 148 & 149 & 166 & 167 & 168 \\
\hline 16 & - & - & - & - & - & - & - & - & - & - & - & - & - & - & - \\
\hline 17 & - & $>$ & $>$ & $>$ & - & - & - & $>$ & - & - & - & $>$ & $>$ & - & - \\
\hline 18 & - & - & - & - & - & - & - & $>$ & - & - & - & $>$ & - & - & - \\
\hline 19 & - & - & - & - & - & - & - & - & - & - & - & - & - & - & - \\
\hline 20 & - & - & - & - & - & - & - & - & - & - & - & - & - & - & - \\
\hline 21 & - & - & - & - & - & - & - & - & - & - & - & - & - & - & - \\
\hline 22 & - & - & - & - & - & - & $>$ & $>$ & - & - & $>$ & $>$ & - & - & - \\
\hline 23 & - & - & - & - & - & - & - & - & - & - & - & - & - & - & - \\
\hline 24 & - & - & - & - & - & - & - & - & - & - & - & - & - & - & - \\
\hline 25 & - & - & - & - & - & - & - & - & - & - & - & - & - & - & - \\
\hline 26 & - & - & - & - & - & - & - & - & - & - & - & - & - & - & - \\
\hline 27 & - & - & - & - & - & - & - & - & - & - & - & - & - & - & - \\
\hline 28 & - & - & - & - & - & - & - & - & - & - & - & - & - & - & - \\
\hline 29 & - & - & - & - & - & - & - & - & - & - & - & - & - & - & - \\
\hline 30 & - & - & - & - & - & - & - & - & - & - & - & - & $>$ & $>$ & $>$ \\
\hline 31 & - & - & - & - & - & - & - & - & - & - & - & - & - & - & - \\
\hline 32 & - & - & - & - & - & - & - & - & - & - & - & - & - & - & - \\
\hline 33 & - & $>$ & $>$ & $>$ & $>$ & $>$ & $>$ & $>$ & $>$ & $>$ & $>$ & $>$ & $>$ & $>$ & $>$ \\
\hline 34 & - & $>$ & $>$ & $>$ & $>$ & $>$ & $>$ & $>$ & $>$ & $>$ & $>$ & $>$ & $>$ & $>$ & $>$ \\
\hline 35 & - & - & - & - & - & - & - & - & - & - & - & - & - & - & - \\
\hline 36 & - & - & - & - & - & - & - & - & - & - & - & - & - & - & $<$ \\
\hline 37 & - & - & - & - & - & - & - & - & - & - & - & - & - & - & - \\
\hline 38 & - & - & - & - & - & - & - & - & - & - & - & - & - & - & - \\
\hline 39 & - & - & - & - & - & - & - & - & - & - & - & - & - & - & - \\
\hline 40 & - & - & - & - & - & - & - & - & - & - & - & - & - & - & - \\
\hline 41 & - & - & - & - & - & - & - & - & - & - & - & - & - & - & - \\
\hline 42 & - & - & - & - & - & - & - & - & - & - & - & - & - & - & - \\
\hline 43 & - & - & - & - & - & - & - & - & - & - & - & - & - & - & - \\
\hline 44 & - & - & - & - & - & - & - & - & - & - & - & - & - & - & - \\
\hline 45 & - & - & - & - & - & - & - & - & - & - & - & - & - & - & - \\
\hline 46 & - & - & - & - & - & - & - & - & - & - & - & - & - & - & - \\
\hline 47 & - & - & - & - & - & - & - & - & - & - & - & - & - & - & - \\
\hline 48 & - & - & - & - & - & - & - & - & - & - & - & - & - & - & - \\
\hline 49 & - & - & - & - & - & - & - & $>$ & - & - & - & $>$ & - & - & - \\
\hline 50 & - & - & - & - & - & - & - & $>$ & - & - & - & $>$ & - & - & - \\
\hline 51 & - & - & - & - & - & - & - & - & - & - & - & - & - & - & - \\
\hline 52 & - & - & - & - & - & - & - & - & - & - & - & - & - & - & - \\
\hline
\end{tabular}

(Continued on next page) 
Table 11 (Continued from previous page)

\begin{tabular}{|c|c|c|c|c|c|c|c|c|c|c|c|c|c|c|c|}
\hline & 138 & 139 & 140 & 141 & 142 & 143 & 144 & 145 & 146 & 147 & 148 & 149 & 166 & 167 & 168 \\
\hline 53 & - & - & - & - & - & - & - & - & - & - & - & - & - & - & - \\
\hline 54 & - & - & - & - & - & - & - & - & - & - & - & - & - & - & - \\
\hline 55 & - & - & - & - & - & - & - & - & - & - & - & - & - & - & - \\
\hline 56 & - & - & - & - & - & - & - & - & - & - & - & - & - & - & - \\
\hline 57 & - & - & - & - & - & - & - & - & - & - & - & - & - & - & - \\
\hline 58 & - & - & - & - & - & - & - & - & - & - & - & - & - & - & - \\
\hline 59 & - & - & - & - & - & - & - & - & - & - & - & - & - & - & - \\
\hline 60 & - & - & - & - & - & - & - & - & - & - & - & - & - & - & - \\
\hline 61 & - & - & - & - & - & - & - & - & - & - & - & - & - & - & - \\
\hline 62 & - & - & - & - & - & - & - & - & - & - & - & - & - & - & - \\
\hline 63 & - & - & - & - & - & - & - & - & - & - & - & - & - & - & - \\
\hline 64 & - & - & - & - & - & - & - & - & - & - & - & - & - & - & - \\
\hline 65 & - & - & - & - & - & - & - & - & - & - & - & - & - & - & - \\
\hline 66 & - & - & - & - & - & - & - & - & - & - & - & - & - & - & - \\
\hline 67 & - & - & - & - & - & - & - & - & - & - & - & - & - & - & - \\
\hline 68 & - & - & - & - & - & - & - & - & - & - & - & - & - & - & - \\
\hline 69 & - & - & - & - & - & - & - & - & - & - & - & - & - & - & - \\
\hline 70 & - & - & - & - & - & - & - & - & - & - & - & - & - & - & - \\
\hline 71 & - & $>$ & $>$ & $>$ & - & - & - & $>$ & - & - & - & $>$ & $>$ & - & - \\
\hline 72 & - & - & - & - & - & - & - & $>$ & - & - & - & $>$ & - & - & - \\
\hline 73 & - & - & - & - & - & - & - & - & - & - & - & - & - & - & - \\
\hline 74 & - & - & - & - & - & - & - & - & - & - & - & - & - & - & - \\
\hline 75 & - & - & - & - & - & - & - & - & - & - & - & - & - & - & - \\
\hline 76 & - & - & - & - & - & $>$ & - & $>$ & - & $>$ & - & $>$ & - & $>$ & - \\
\hline 77 & - & - & - & - & - & - & - & - & - & - & - & - & - & - & - \\
\hline 78 & - & - & - & - & - & - & - & - & - & - & - & - & - & - & - \\
\hline 79 & - & - & - & - & - & - & - & - & - & - & - & - & - & - & - \\
\hline 80 & - & - & - & - & - & - & - & - & - & - & - & - & - & - & - \\
\hline 81 & - & - & - & - & - & - & - & - & - & - & - & - & - & - & - \\
\hline 82 & - & - & - & - & - & - & - & - & - & - & - & - & - & - & - \\
\hline 83 & - & - & - & - & - & - & - & - & - & - & - & - & - & - & - \\
\hline 84 & - & - & - & - & - & - & - & - & - & - & - & - & - & - & - \\
\hline 85 & - & - & - & - & - & - & - & - & - & - & - & - & - & - & - \\
\hline 86 & - & - & - & - & - & - & - & - & - & - & - & - & - & - & - \\
\hline 87 & - & - & - & - & - & - & - & - & - & - & - & - & - & - & - \\
\hline 88 & - & - & - & - & - & - & - & - & - & - & - & - & - & - & - \\
\hline 89 & - & - & - & - & - & - & - & - & - & - & - & - & - & - & - \\
\hline
\end{tabular}

(Continued on next page) 
Table 11 (Continued from previous page)

\begin{tabular}{|c|c|c|c|c|c|c|c|c|c|c|c|c|c|c|c|}
\hline & 138 & 139 & 140 & 141 & 142 & 143 & 144 & 145 & 146 & 147 & 148 & 149 & 166 & 167 & 168 \\
\hline 90 & - & - & - & - & - & - & - & - & - & - & - & - & - & - & - \\
\hline 91 & - & - & - & - & - & - & - & - & - & - & - & - & - & - & - \\
\hline 92 & - & - & - & - & - & - & - & - & - & - & - & - & - & - & - \\
\hline 93 & - & - & - & - & - & - & - & - & - & - & - & - & - & - & - \\
\hline 94 & - & - & - & - & - & - & - & - & - & - & - & - & - & - & - \\
\hline 95 & - & - & - & - & - & - & - & - & - & - & - & - & - & - & - \\
\hline 96 & - & - & - & - & - & - & - & - & - & - & - & - & - & - & - \\
\hline 97 & - & - & - & - & - & - & - & - & - & - & - & - & - & - & - \\
\hline 98 & - & - & - & - & - & - & - & - & - & - & - & - & - & - & - \\
\hline 99 & - & - & - & - & - & - & - & - & - & - & - & - & - & - & - \\
\hline 100 & - & - & - & - & - & - & - & - & - & - & - & - & - & - & - \\
\hline 101 & - & - & - & - & - & - & - & - & - & - & - & - & - & - & - \\
\hline 102 & - & - & - & - & - & - & - & - & - & - & - & - & - & - & - \\
\hline 103 & - & - & - & - & - & - & - & - & - & - & - & - & - & - & - \\
\hline 104 & - & - & - & - & - & - & - & - & - & - & - & - & - & - & - \\
\hline 105 & - & - & - & - & - & - & - & - & - & - & - & - & - & - & - \\
\hline 106 & - & - & - & - & - & - & - & - & - & - & - & - & - & - & - \\
\hline 107 & - & - & - & - & - & - & - & - & - & - & - & - & - & - & - \\
\hline 108 & - & - & - & - & - & - & - & - & - & - & - & - & - & - & - \\
\hline 109 & - & - & - & - & - & - & - & - & - & - & - & - & - & - & - \\
\hline 110 & - & $>$ & $>$ & $>$ & - & - & - & $>$ & - & - & - & $>$ & $>$ & - & - \\
\hline 111 & - & - & - & - & - & - & - & - & - & - & - & - & - & - & - \\
\hline 112 & - & $>$ & $>$ & $>$ & - & - & - & $>$ & - & - & - & $>$ & $>$ & - & - \\
\hline 113 & - & - & - & - & - & - & - & - & - & - & - & - & - & - & - \\
\hline 114 & - & - & - & - & - & - & - & - & - & - & - & - & - & - & - \\
\hline 115 & - & $>$ & $>$ & $>$ & $>$ & $>$ & $>$ & $>$ & $>$ & $>$ & $>$ & $>$ & $>$ & $>$ & $>$ \\
\hline 116 & - & - & - & - & - & - & - & - & - & - & - & - & - & - & - \\
\hline 117 & - & - & - & - & - & - & - & - & - & - & - & - & - & - & - \\
\hline 118 & - & - & - & - & - & - & - & - & - & - & - & - & - & - & - \\
\hline 119 & - & - & - & - & - & - & - & - & - & - & - & - & - & - & - \\
\hline 120 & - & - & - & - & - & - & - & $>$ & - & - & - & $>$ & - & - & - \\
\hline 121 & - & - & - & - & - & - & - & - & - & - & - & - & - & - & - \\
\hline 122 & - & - & - & - & - & - & - & $>$ & - & - & - & $>$ & - & - & - \\
\hline 123 & - & - & - & - & - & - & - & - & - & - & - & - & - & - & - \\
\hline 124 & - & - & - & - & - & - & - & - & - & - & - & - & - & - & - \\
\hline 125 & - & $>$ & $>$ & $>$ & - & - & - & $>$ & - & - & - & $>$ & $>$ & - & - \\
\hline 126 & - & - & - & - & - & - & - & - & - & - & - & - & - & - & - \\
\hline
\end{tabular}

(Continued on next page) 
Table 11 (Continued from previous page)

\begin{tabular}{|c|c|c|c|c|c|c|c|c|c|c|c|c|c|c|c|}
\hline & 138 & 139 & 140 & 141 & 142 & 143 & 144 & 145 & 146 & 147 & 148 & 149 & 166 & 167 & 168 \\
\hline 127 & - & $>$ & $>$ & $>$ & - & - & - & $>$ & - & - & - & $>$ & $>$ & - & - \\
\hline 128 & - & - & - & - & - & - & - & - & - & - & - & - & - & - & - \\
\hline 129 & - & - & - & - & - & - & - & - & - & - & - & - & - & - & - \\
\hline 130 & - & $>$ & $>$ & $>$ & $>$ & $>$ & $>$ & $>$ & $>$ & $>$ & $>$ & $>$ & $>$ & $>$ & $>$ \\
\hline 131 & - & - & - & - & - & - & - & - & - & - & - & - & - & - & - \\
\hline 132 & - & - & - & - & - & - & - & - & - & - & - & - & - & - & - \\
\hline 133 & - & - & - & - & - & - & - & - & - & - & - & - & - & - & - \\
\hline 134 & - & - & - & - & - & - & - & - & - & - & - & - & - & - & - \\
\hline 135 & - & - & - & - & - & - & - & $>$ & - & - & - & $>$ & - & - & - \\
\hline 136 & - & - & - & - & - & - & - & - & - & - & - & - & - & - & - \\
\hline 137 & - & - & - & - & - & - & - & $>$ & - & - & - & $>$ & - & - & - \\
\hline 138 & $=$ & - & - & - & - & - & - & - & - & - & - & - & - & - & - \\
\hline 139 & - & $=$ & $>$ & $<$ & - & - & - & $>$ & $<$ & - & - & - & - & - & - \\
\hline 140 & - & $<$ & $=$ & $<$ & $<$ & - & - & $>$ & $<$ & - & - & - & - & - & - \\
\hline 141 & - & $>$ & $>$ & $=$ & - & - & - & $>$ & $<$ & - & - & $>$ & - & - & - \\
\hline 142 & - & - & $>$ & - & $=$ & $>$ & $>$ & $>$ & $<$ & - & - & - & - & - & - \\
\hline 143 & - & - & - & - & $<$ & $=$ & - & $>$ & $<$ & $<$ & - & - & - & - & - \\
\hline 144 & - & - & - & - & $<$ & - & $=$ & $>$ & $<$ & - & $<$ & - & - & - & - \\
\hline 145 & - & $<$ & $<$ & $<$ & $<$ & $<$ & $<$ & $=$ & $<$ & $<$ & $<$ & $<$ & - & - & - \\
\hline 146 & - & $>$ & $>$ & $>$ & $>$ & $>$ & $>$ & $>$ & $=$ & $>$ & $>$ & $>$ & - & - & - \\
\hline 147 & - & - & - & - & - & $>$ & - & $>$ & $<$ & $=$ & - & $>$ & - & - & - \\
\hline 148 & - & - & - & - & - & - & $>$ & $>$ & $<$ & - & $=$ & $>$ & - & - & - \\
\hline 149 & - & - & - & $<$ & - & - & - & $>$ & $<$ & $<$ & $<$ & $=$ & - & - & - \\
\hline 166 & - & - & - & - & - & - & - & - & - & - & - & - & $=$ & $<$ & $<$ \\
\hline 167 & - & - & - & - & - & - & - & - & - & - & - & - & $>$ & $=$ & $<$ \\
\hline 168 & - & - & - & - & - & - & - & - & - & - & - & - & $>$ & $>$ & $=$ \\
\hline 169 & - & - & - & - & - & - & - & - & - & - & - & - & $>$ & - & - \\
\hline 170 & - & - & - & - & - & - & - & - & - & - & - & - & $>$ & - & - \\
\hline 171 & - & - & - & - & - & - & - & - & - & - & - & - & $>$ & - & - \\
\hline 172 & - & - & - & - & - & - & - & - & - & - & - & - & $>$ & - & - \\
\hline 173 & - & - & - & - & - & - & - & - & - & - & - & - & $>$ & - & - \\
\hline 174 & - & - & - & - & - & - & - & - & - & - & - & - & $>$ & - & - \\
\hline 175 & - & - & - & - & - & - & - & - & - & - & - & - & $>$ & - & - \\
\hline 176 & - & $>$ & $>$ & $>$ & - & - & - & $>$ & - & - & - & $>$ & $>$ & - & - \\
\hline 177 & - & - & - & - & - & $>$ & - & $>$ & - & $>$ & - & $>$ & $>$ & $>$ & - \\
\hline 178 & - & $>$ & $>$ & $>$ & $>$ & $>$ & $>$ & $>$ & $>$ & $>$ & $>$ & $>$ & $>$ & $>$ & $>$ \\
\hline 179 & - & - & - & - & - & - & - & $>$ & - & - & - & $>$ & $>$ & - & - \\
\hline
\end{tabular}

(Continued on next page) 
Table 11 (Continued from previous page)

\begin{tabular}{|c|c|c|c|c|c|c|c|c|c|c|c|c|c|c|c|}
\hline & 138 & 139 & 140 & 141 & 142 & 143 & 144 & 145 & 146 & 147 & 148 & 149 & 166 & 167 & 168 \\
\hline 180 & - & - & - & - & - & - & $>$ & $>$ & - & - & $>$ & $>$ & $>$ & - & - \\
\hline 181 & - & - & - & - & - & - & - & - & - & - & - & - & $>$ & - & - \\
\hline 182 & - & - & - & - & - & - & - & - & - & - & - & - & - & - & - \\
\hline 183 & - & - & - & - & - & - & - & - & - & - & - & - & - & - & - \\
\hline 184 & - & - & - & - & - & - & - & - & - & - & - & - & - & - & - \\
\hline 185 & - & - & - & - & - & - & - & - & - & - & - & - & - & - & - \\
\hline 186 & - & $>$ & $>$ & $>$ & - & - & - & $>$ & - & - & - & $>$ & - & - & - \\
\hline 187 & - & - & - & - & - & - & $>$ & $>$ & - & - & $>$ & $>$ & - & - & - \\
\hline 188 & - & $>$ & $>$ & $>$ & $>$ & $>$ & $>$ & $>$ & $>$ & $>$ & $>$ & $>$ & - & $>$ & $>$ \\
\hline 189 & - & - & - & - & - & - & - & $>$ & - & - & - & $>$ & - & - & - \\
\hline 190 & - & - & - & - & - & - & $>$ & $>$ & - & - & $>$ & $>$ & - & - & - \\
\hline 191 & - & - & - & - & - & - & - & - & - & - & - & - & - & - & - \\
\hline 192 & - & - & - & - & - & - & - & - & - & - & - & - & $>$ & - & - \\
\hline 193 & - & - & - & - & - & - & - & - & - & - & - & - & - & - & - \\
\hline 194 & - & - & - & - & - & - & - & - & - & - & - & - & $>$ & - & - \\
\hline 195 & - & - & - & - & - & - & - & - & - & - & - & - & - & - & - \\
\hline 196 & - & - & - & - & - & - & - & - & - & - & - & - & - & - & - \\
\hline 197 & - & - & - & - & - & - & - & - & - & - & - & - & - & - & - \\
\hline 198 & - & - & - & - & - & - & - & - & - & - & - & - & - & - & - \\
\hline
\end{tabular}

Table 12. Relations between the intuitionistic fuzzy implications $\rightarrow_{169}, \ldots, \rightarrow_{183}$

\begin{tabular}{|c|c|c|c|c|c|c|c|c|c|c|c|c|c|c|c|}
\hline & 169 & 170 & 171 & 172 & 173 & 174 & 175 & 176 & 177 & 178 & 179 & 180 & 181 & 182 & 183 \\
\hline 1 & - & - & - & - & - & - & - & $<$ & - & $<$ & - & - & - & - & - \\
\hline 2 & - & - & - & $>$ & - & $>$ & - & $<$ & - & $<$ & - & - & - & - & - \\
\hline 3 & - & - & - & $>$ & - & $>$ & - & $<$ & - & $<$ & - & - & $>$ & $>$ & - \\
\hline 4 & $>$ & - & - & - & - & - & - & $<$ & - & $<$ & - & - & - & - & - \\
\hline 5 & $>$ & - & - & - & - & $>$ & - & - & - & - & $>$ & - & - & - & - \\
\hline 6 & - & - & - & - & - & - & - & - & - & - & - & - & - & - & - \\
\hline 7 & - & - & - & - & - & - & - & $<$ & - & $<$ & - & - & - & - & - \\
\hline 8 & - & - & - & $>$ & - & $>$ & - & - & - & $<$ & - & - & - & - & - \\
\hline 9 & - & - & - & - & - & - & - & - & - & - & - & - & - & - & - \\
\hline 10 & - & - & - & - & - & - & - & $<$ & - & $<$ & - & - & - & - & - \\
\hline 11 & - & - & - & $>$ & - & $>$ & - & - & - & $<$ & - & - & $>$ & $>$ & - \\
\hline 12 & $>$ & - & - & - & - & - & - & $<$ & $<$ & $<$ & $<$ & $<$ & - & - & - \\
\hline 13 & $>$ & - & - & - & - & - & - & - & - & - & - & - & - & - & - \\
\hline 14 & - & - & - & - & - & $>$ & - & - & - & $<$ & - & $<$ & - & - & - \\
\hline 15 & - & - & - & - & $<$ & $>$ & - & $<$ & $<$ & $<$ & - & $<$ & - & - & - \\
\hline
\end{tabular}


Table 12 (Continued from previous page)

\begin{tabular}{|c|c|c|c|c|c|c|c|c|c|c|c|c|c|c|c|}
\hline & 169 & 170 & 171 & 172 & 173 & 174 & 175 & 176 & 177 & 178 & 179 & 180 & 181 & 182 & 183 \\
\hline 16 & - & - & - & - & - & - & - & $<$ & - & $<$ & - & - & $"="$ & $>$ & $<$ \\
\hline 17 & $>$ & - & - & - & - & - & - & - & - & - & - & - & - & - & - \\
\hline 18 & $>$ & - & - & - & - & - & - & $<$ & - & $<$ & - & $<$ & - & - & - \\
\hline 19 & - & - & - & - & - & - & - & $<$ & - & $<$ & - & $<$ & $<$ & - & $<$ \\
\hline 20 & - & - & - & $>$ & - & $>$ & - & - & - & - & - & - & - & $>$ & - \\
\hline 21 & - & - & - & - & - & - & - & - & - & - & - & - & - & - & - \\
\hline 22 & $>$ & $>$ & - & - & - & - & - & - & - & $<$ & - & $<$ & - & - & - \\
\hline 23 & - & - & $>$ & - & - & $>$ & $>$ & - & - & - & - & - & - & - & - \\
\hline 24 & - & - & - & - & $<$ & $>$ & - & - & - & $<$ & - & - & - & - & - \\
\hline 25 & - & - & - & - & - & - & - & $<$ & - & $<$ & - & - & - & - & - \\
\hline 26 & - & - & - & - & - & - & - & $<$ & - & $<$ & - & - & $<$ & - & $<$ \\
\hline 27 & - & - & - & - & - & $>$ & - & - & - & - & - & - & - & - & - \\
\hline 28 & - & - & - & - & - & - & - & $<$ & - & $<$ & - & - & - & - & - \\
\hline 29 & - & - & - & - & - & - & - & - & - & - & - & - & - & - & - \\
\hline 30 & - & - & - & - & - & - & - & - & - & $<$ & - & - & - & - & - \\
\hline 31 & - & - & $>$ & $>$ & $>$ & $>$ & $>$ & - & - & $<$ & - & - & - & - & - \\
\hline 32 & - & - & $>$ & $>$ & $>$ & $>$ & $>$ & - & - & $<$ & - & - & $>$ & $>$ & $>$ \\
\hline 33 & $>$ & $>$ & - & - & - & - & - & - & - & $<$ & - & - & $>$ & $>$ & $>$ \\
\hline 34 & $>$ & $>$ & $>$ & $>$ & $>$ & $>$ & $>$ & $>$ & $>$ & $>$ & $>$ & $>$ & $>$ & $>$ & $>$ \\
\hline 35 & - & - & - & - & - & - & - & - & - & - & - & - & - & - & - \\
\hline 36 & - & - & - & - & - & - & - & - & - & $<$ & - & - & - & - & - \\
\hline 37 & - & - & $>$ & $>$ & $>$ & $>$ & $>$ & - & - & $<$ & - & - & - & - & - \\
\hline 38 & - & - & - & - & - & - & - & - & - & - & - & - & - & - & - \\
\hline 39 & - & - & - & - & - & - & - & - & - & $<$ & - & - & - & - & - \\
\hline 40 & - & - & $>$ & $>$ & $"="$ & $>$ & $>$ & - & - & $<$ & - & - & - & - & - \\
\hline 41 & - & - & - & - & - & - & - & - & - & $<$ & - & - & $>$ & $>$ & $"="$ \\
\hline 42 & - & - & $>$ & $>$ & $>$ & $>$ & $>$ & - & - & - & - & - & $>$ & $>$ & $>$ \\
\hline 43 & - & - & - & - & - & - & - & - & - & $<$ & - & - & $>$ & $>$ & $>$ \\
\hline 44 & - & - & - & - & - & - & - & - & - & $<$ & - & - & $>$ & $>$ & $>$ \\
\hline 45 & - & - & - & - & - & - & - & - & - & - & - & - & - & - & - \\
\hline 46 & $>$ & - & - & - & - & - & - & $<$ & $<$ & $<$ & $<$ & $<$ & - & - & - \\
\hline 47 & - & - & - & - & - & $>$ & - & $<$ & $<$ & $<$ & $<$ & $<$ & - & - & - \\
\hline 48 & - & - & - & - & - & $>$ & - & $<$ & $<$ & $<$ & $<$ & $<$ & - & - & - \\
\hline 49 & $>$ & - & - & - & - & $>$ & - & - & - & - & $>$ & - & - & - & - \\
\hline 50 & $>$ & - & - & - & - & - & - & - & - & - & - & - & - & - & - \\
\hline 51 & $<$ & $<$ & - & - & - & - & - & $<$ & $<$ & $<$ & $<$ & $<$ & - & - & - \\
\hline 52 & - & - & - & - & - & $>$ & - & $<$ & $<$ & $<$ & $<$ & $<$ & - & - & - \\
\hline \multicolumn{16}{|c|}{ (Continued on next page) } \\
\hline
\end{tabular}


Table 12 (Continued from previous page)

\begin{tabular}{|c|c|c|c|c|c|c|c|c|c|c|c|c|c|c|c|}
\hline & 169 & 170 & 171 & 172 & 173 & 174 & 175 & 176 & 177 & 178 & 179 & 180 & 181 & 182 & 183 \\
\hline 53 & - & - & - & - & - & - & - & - & - & - & - & - & - & - & - \\
\hline 54 & - & - & - & - & - & - & - & $<$ & $<$ & $<$ & $<$ & $<$ & - & - & - \\
\hline 55 & - & - & $<$ & $<$ & $<$ & $"="$ & $<$ & $<$ & $<$ & $<$ & $<$ & $<$ & - & - & - \\
\hline 56 & - & - & - & - & - & - & - & $<$ & $<$ & $<$ & $<$ & $<$ & $<$ & $<$ & $<$ \\
\hline 57 & - & - & - & - & - & $>$ & - & - & - & - & - & - & - & - & - \\
\hline 58 & - & - & - & - & - & - & - & $<$ & $<$ & $<$ & $<$ & $<$ & - & - & - \\
\hline 59 & - & - & - & - & - & - & - & $<$ & $<$ & $<$ & $<$ & $<$ & - & - & - \\
\hline 60 & - & - & - & - & - & - & - & - & - & - & - & - & - & - & - \\
\hline 61 & - & - & - & - & - & - & - & $<$ & - & $<$ & - & - & - & - & - \\
\hline 62 & - & - & - & - & - & $>$ & $>$ & - & - & $<$ & - & - & - & - & - \\
\hline 63 & - & - & - & - & - & $>$ & $>$ & - & - & $<$ & - & - & - & - & - \\
\hline 64 & - & - & - & - & - & - & - & - & - & - & - & - & - & - & - \\
\hline 65 & - & - & - & - & - & $>$ & $>$ & - & - & $<$ & - & - & - & - & - \\
\hline 66 & - & - & - & - & - & - & - & - & - & - & - & - & - & - & - \\
\hline 67 & - & - & - & - & - & - & - & $<$ & - & $<$ & - & - & - & - & - \\
\hline 68 & - & - & - & - & - & $>$ & $>$ & - & - & $<$ & - & - & - & - & - \\
\hline 69 & - & - & - & - & - & $>$ & - & - & $<$ & $<$ & - & - & - & - & - \\
\hline 70 & - & - & - & - & - & - & - & - & - & $<$ & - & - & - & - & - \\
\hline 71 & $>$ & - & - & - & - & - & - & - & - & - & - & - & - & - & - \\
\hline 72 & $>$ & - & - & - & - & - & - & $<$ & $<$ & $<$ & - & - & - & - & - \\
\hline 73 & - & - & - & - & - & - & - & $<$ & $<$ & $<$ & - & - & - & - & - \\
\hline 74 & - & - & - & - & - & $>$ & $>$ & - & - & - & - & - & - & - & - \\
\hline 75 & - & - & - & - & - & - & - & - & - & - & - & - & - & - & - \\
\hline 76 & $>$ & - & - & - & - & - & - & - & $<$ & $<$ & - & - & - & $>$ & - \\
\hline 77 & - & - & $>$ & $>$ & - & $>$ & - & - & - & - & - & - & - & - & - \\
\hline 78 & - & - & - & - & - & - & - & $<$ & - & $<$ & - & - & - & - & - \\
\hline 79 & - & - & - & - & - & $>$ & - & - & - & - & - & - & - & - & - \\
\hline 80 & - & - & - & - & - & - & - & $<$ & - & $<$ & - & - & - & - & - \\
\hline 81 & - & - & - & - & - & - & - & - & - & - & - & - & - & - & - \\
\hline 82 & - & - & - & - & - & - & - & - & - & $<$ & - & - & - & - & - \\
\hline 83 & - & - & $>$ & $>$ & $>$ & $>$ & $>$ & - & - & $<$ & - & - & - & - & - \\
\hline 84 & - & - & $>$ & $>$ & $>$ & $>$ & $>$ & - & - & $<$ & - & - & - & - & - \\
\hline 85 & - & - & - & - & - & - & - & - & - & - & - & - & - & - & - \\
\hline 86 & - & - & - & - & - & - & - & - & - & $<$ & - & - & $>$ & $>$ & $>$ \\
\hline 87 & - & - & - & - & - & - & - & - & - & $<$ & - & - & - & - & - \\
\hline 88 & - & - & $>$ & $>$ & $>$ & $>$ & $>$ & - & - & - & - & - & - & - & - \\
\hline 89 & - & - & - & - & - & - & - & - & - & $<$ & - & - & - & - & - \\
\hline
\end{tabular}


Table 12 (Continued from previous page)

\begin{tabular}{|c|c|c|c|c|c|c|c|c|c|c|c|c|c|c|c|}
\hline & 169 & 170 & 171 & 172 & 173 & 174 & 175 & 176 & 177 & 178 & 179 & 180 & 181 & 182 & 183 \\
\hline 90 & - & - & - & - & - & - & - & - & - & - & - & - & - & - & - \\
\hline 91 & - & - & - & - & - & - & - & $<$ & $<$ & $<$ & $<$ & $<$ & - & - & - \\
\hline 92 & - & - & - & - & - & $>$ & - & $<$ & $<$ & $<$ & $<$ & $<$ & - & - & - \\
\hline 93 & - & - & - & - & - & $>$ & - & $<$ & $<$ & $<$ & $<$ & $<$ & - & - & - \\
\hline 94 & - & - & - & - & - & - & - & - & - & - & - & - & - & - & - \\
\hline 95 & - & - & - & - & - & - & - & $<$ & $<$ & $<$ & $<$ & $<$ & - & - & - \\
\hline 96 & - & - & - & - & - & - & - & $<$ & $<$ & $<$ & $<$ & $<$ & - & - & - \\
\hline 97 & - & - & - & - & - & $>$ & - & - & - & - & - & - & - & - & - \\
\hline 98 & - & - & - & - & - & - & - & $<$ & $<$ & $<$ & $<$ & $<$ & - & - & - \\
\hline 99 & - & - & - & - & - & - & - & - & - & - & - & - & - & - & - \\
\hline 100 & - & - & - & - & - & - & - & - & - & - & - & - & - & - & - \\
\hline 101 & - & - & - & - & - & - & - & - & - & - & - & - & - & - & - \\
\hline 102 & - & - & - & - & - & - & - & - & - & - & - & - & - & - & - \\
\hline 103 & - & - & - & - & - & - & - & - & - & - & - & - & - & - & - \\
\hline 104 & - & - & - & - & - & - & - & - & - & - & - & - & - & - & - \\
\hline 105 & - & - & - & - & - & - & - & - & - & - & - & - & - & - & - \\
\hline 106 & - & - & - & - & - & - & - & - & - & - & - & - & - & - & - \\
\hline 107 & - & - & - & - & - & - & - & - & - & - & - & - & - & - & - \\
\hline 108 & - & - & - & - & - & - & - & - & - & - & - & - & - & - & - \\
\hline 109 & - & - & - & - & - & - & - & - & - & - & - & - & - & - & - \\
\hline 110 & $>$ & - & - & - & - & - & - & - & - & - & - & - & - & - & - \\
\hline 111 & - & - & - & - & - & - & - & - & - & - & - & - & - & - & - \\
\hline 112 & $>$ & - & - & - & - & - & - & - & - & - & - & - & - & - & - \\
\hline 113 & - & - & - & - & - & - & - & - & - & - & - & - & - & - & - \\
\hline 114 & - & - & - & - & - & - & - & - & - & - & - & - & - & - & - \\
\hline 115 & $>$ & $>$ & - & - & - & - & - & - & - & - & - & - & $>$ & $>$ & $>$ \\
\hline 116 & - & - & - & - & - & - & - & - & - & - & - & - & - & - & - \\
\hline 117 & - & - & - & - & - & - & - & - & - & - & - & - & - & - & - \\
\hline 118 & - & - & - & - & - & - & - & - & - & - & - & - & - & - & - \\
\hline 119 & - & - & - & - & - & - & - & - & - & - & - & - & - & - & - \\
\hline 120 & $>$ & - & - & - & - & - & - & - & - & - & - & - & - & - & - \\
\hline 121 & - & - & - & - & - & - & - & - & - & - & - & - & - & - & - \\
\hline 122 & $>$ & - & - & - & - & - & - & - & - & - & - & - & - & - & - \\
\hline 123 & - & - & - & - & - & - & - & - & - & - & - & - & - & - & - \\
\hline 124 & - & - & - & - & - & - & - & - & - & - & - & - & - & - & - \\
\hline 125 & $>$ & - & - & - & - & - & - & - & - & - & - & - & - & - & - \\
\hline 126 & - & - & - & - & - & - & - & - & - & - & - & - & - & - & - \\
\hline \multicolumn{16}{|c|}{ (Continued on next page) } \\
\hline
\end{tabular}


Table 12 (Continued from previous page)

\begin{tabular}{|c|c|c|c|c|c|c|c|c|c|c|c|c|c|c|c|}
\hline & 169 & 170 & 171 & 172 & 173 & 174 & 175 & 176 & 177 & 178 & 179 & 180 & 181 & 182 & 183 \\
\hline 127 & $>$ & - & - & - & - & - & - & - & - & - & - & - & - & - & - \\
\hline 128 & - & - & - & - & - & - & - & - & - & - & - & - & - & - & - \\
\hline 129 & - & - & - & - & - & - & - & - & - & - & - & - & - & - & - \\
\hline 130 & $>$ & $>$ & - & - & - & - & - & - & - & - & - & - & $>$ & $>$ & $>$ \\
\hline 131 & - & - & - & - & - & - & - & - & - & - & - & - & $>$ & $>$ & $>$ \\
\hline 132 & - & - & - & - & - & - & - & - & - & - & - & - & - & - & - \\
\hline 133 & - & - & - & - & - & - & - & - & - & - & - & - & - & - & - \\
\hline 134 & - & - & - & - & - & - & - & - & - & - & - & - & - & - & - \\
\hline 135 & $>$ & - & - & - & - & - & - & - & - & - & - & - & - & - & - \\
\hline 136 & - & - & - & - & - & - & - & - & - & - & - & - & - & - & - \\
\hline 137 & $>$ & - & - & - & - & - & - & - & - & - & - & - & - & - & - \\
\hline 138 & - & - & - & - & - & - & - & - & - & - & - & - & - & - & - \\
\hline 139 & - & - & - & - & - & - & - & $<$ & - & $<$ & - & - & - & - & - \\
\hline 140 & - & - & - & - & - & - & - & $<$ & - & $<$ & - & - & - & - & - \\
\hline 141 & - & - & - & - & - & - & - & $<$ & - & $<$ & - & - & - & - & - \\
\hline 142 & - & - & - & - & - & - & - & - & - & $<$ & - & - & - & - & - \\
\hline 143 & - & - & - & - & - & - & - & - & $<$ & $<$ & - & - & - & - & - \\
\hline 144 & - & - & - & - & - & - & - & - & - & $<$ & - & $<$ & - & - & - \\
\hline 145 & - & - & - & - & - & - & - & $<$ & $<$ & $<$ & $<$ & $<$ & - & - & - \\
\hline 146 & - & - & - & - & - & - & - & - & - & $<$ & - & - & - & - & - \\
\hline 147 & - & - & - & - & - & - & - & - & $<$ & $<$ & - & - & - & - & - \\
\hline 148 & - & - & - & - & - & - & - & - & - & $<$ & - & $<$ & - & - & - \\
\hline 149 & - & - & - & - & - & - & - & $<$ & $<$ & $<$ & $<$ & $<$ & - & - & - \\
\hline 166 & $<$ & $<$ & $<$ & $<$ & $<$ & $<$ & $<$ & $<$ & $<$ & $<$ & $<$ & $<$ & $<$ & - & - \\
\hline 167 & - & - & - & - & - & - & - & - & $<$ & $<$ & - & - & - & - & - \\
\hline 168 & - & - & - & - & - & - & - & - & - & $<$ & - & - & - & - & - \\
\hline 169 & $=$ & $<$ & - & - & - & - & - & $<$ & $<$ & $<$ & $<$ & $<$ & - & - & - \\
\hline 170 & $>$ & $=$ & - & - & - & - & - & - & - & $<$ & - & $<$ & - & - & - \\
\hline 171 & - & - & $=$ & - & $<$ & $>$ & - & - & - & $<$ & - & - & - & - & - \\
\hline 172 & - & - & - & $=$ & $<$ & $>$ & - & $<$ & $<$ & $<$ & - & - & - & - & - \\
\hline 173 & - & - & $>$ & $>$ & $=$ & $>$ & $>$ & - & - & $<$ & - & - & - & - & - \\
\hline 174 & - & - & $<$ & $<$ & $<$ & $=$ & $<$ & $<$ & $<$ & $<$ & $<$ & $<$ & - & - & - \\
\hline 175 & - & - & - & - & $<$ & $>$ & $=$ & - & - & $<$ & - & $<$ & - & - & - \\
\hline 176 & $>$ & - & - & $>$ & - & $>$ & - & $=$ & - & $<$ & $>$ & - & $>$ & $>$ & - \\
\hline 177 & $>$ & - & - & $>$ & - & $>$ & - & - & $=$ & $<$ & $>$ & - & - & $>$ & - \\
\hline 178 & $>$ & $>$ & $>$ & $>$ & $>$ & $>$ & $>$ & $>$ & $>$ & $=$ & $>$ & $>$ & $>$ & $>$ & $>$ \\
\hline 179 & $>$ & - & - & - & - & $>$ & - & $<$ & $<$ & $<$ & $=$ & $<$ & - & - & - \\
\hline \multicolumn{16}{|c|}{ (Continued on next page) } \\
\hline
\end{tabular}


Table 12 (Continued from previous page)

\begin{tabular}{|c|ccccccccccccccc|}
\hline & 169 & 170 & 171 & 172 & 173 & 174 & 175 & 176 & 177 & 178 & 179 & 180 & 181 & 182 & 183 \\
\hline 180 & $>$ & $>$ & - & - & - & $>$ & $>$ & - & - & $<$ & $>$ & $=$ & - & - & - \\
\hline 181 & - & - & - & - & - & - & - & $<$ & - & $<$ & - & - & $=$ & $>$ & $<$ \\
\hline 182 & - & - & - & - & - & - & - & $<$ & $<$ & $<$ & - & - & $<$ & $=$ & $<$ \\
\hline 183 & - & - & - & - & - & - & - & - & - & $<$ & - & - & $>$ & $>$ & $=$ \\
\hline 184 & - & - & - & - & - & - & - & - & - & $<$ & - & $<$ & - & - & $<$ \\
\hline 185 & - & - & - & - & - & - & - & $<$ & $<$ & $<$ & $<$ & $<$ & $<$ & $<$ & $<$ \\
\hline 186 & $>$ & - & - & - & - & $>$ & $>$ & - & - & $<$ & $>$ & - & - & - & - \\
\hline 187 & $>$ & $>$ & - & - & - & $>$ & $>$ & - & - & $<$ & $>$ & $\prime="$ & - & - & - \\
\hline 188 & $>$ & $>$ & $>$ & $>$ & $>$ & $>$ & $>$ & $>$ & $>$ & $="$ & $>$ & $>$ & $>$ & $>$ & $>$ \\
\hline 189 & $>$ & - & - & - & - & $>$ & - & $<$ & $<$ & $<$ & $="$ & $<$ & - & - & - \\
\hline 190 & $>$ & $>$ & - & - & - & $>$ & $>$ & - & - & $<$ & $>$ & $="$ & - & - & - \\
\hline 191 & - & - & - & $>$ & - & $>$ & - & - & - & - & - & - & - & $>$ & - \\
\hline 192 & - & - & - & - & - & - & - & $<$ & - & $<$ & - & - & - & - & - \\
\hline 193 & - & - & - & - & - & - & - & $<$ & $<$ & $<$ & $<$ & $<$ & - & - & - \\
\hline 194 & - & - & - & - & - & - & - & - & - & $<$ & - & - & - & - & - \\
\hline 195 & - & - & - & - & - & - & - & - & $<$ & $<$ & - & - & - & - & - \\
\hline 196 & - & - & - & - & - & - & - & - & - & $<$ & - & - & - & - & - \\
\hline 197 & - & - & - & - & - & - & - & $<$ & $<$ & $<$ & $<$ & $<$ & - & - & - \\
\hline 198 & - & - & - & - & - & - & - & - & - & $<$ & - & $<$ & - & - & - \\
\hline
\end{tabular}

Table 13. Relations between the intuitionistic fuzzy implications $\rightarrow_{184}, \ldots, \rightarrow_{198}$

\begin{tabular}{|c|c|c|c|c|c|c|c|c|c|c|c|c|c|c|c|}
\hline & 184 & 185 & 186 & 187 & 188 & 189 & 190 & 191 & 192 & 193 & 194 & 195 & 196 & 197 & 198 \\
\hline 1 & - & - & - & - & - & - & - & $<$ & - & $<$ & - & - & - & - & - \\
\hline 2 & - & - & - & $>$ & - & $>$ & - & $<$ & - & $<$ & - & - & - & - & - \\
\hline 3 & - & - & - & $>$ & - & $>$ & - & $<$ & - & $<$ & - & - & $>$ & $>$ & - \\
\hline 4 & $>$ & - & - & - & - & - & - & $<$ & - & $<$ & - & - & - & - & - \\
\hline 5 & $>$ & - & - & - & - & $>$ & - & - & - & - & $>$ & - & - & - & - \\
\hline 6 & - & - & - & - & - & - & - & - & - & - & - & - & - & - & - \\
\hline 7 & - & - & - & - & - & - & - & $<$ & - & $<$ & - & - & - & - & - \\
\hline 8 & - & - & - & $>$ & - & $>$ & - & - & - & $<$ & - & - & - & - & - \\
\hline 9 & - & - & - & - & - & - & - & - & - & - & - & - & - & - & - \\
\hline 10 & - & - & - & - & - & - & - & $<$ & - & $<$ & - & - & - & - & - \\
\hline 11 & - & - & - & $>$ & - & $>$ & - & - & - & $<$ & - & - & $>$ & $>$ & - \\
\hline 12 & $>$ & - & - & - & - & - & - & $<$ & $<$ & $<$ & $<$ & $<$ & - & - & - \\
\hline 13 & $>$ & - & - & - & - & - & - & - & - & - & - & - & - & - & - \\
\hline 14 & - & - & - & - & - & $>$ & - & - & - & $<$ & - & $<$ & - & - & - \\
\hline 15 & - & - & - & - & $<$ & $>$ & - & $<$ & $<$ & $<$ & - & $<$ & - & - & - \\
\hline
\end{tabular}


Table 13 (Continued from previous page)

\begin{tabular}{|c|c|c|c|c|c|c|c|c|c|c|c|c|c|c|c|}
\hline & 169 & 170 & 171 & 172 & 173 & 174 & 175 & 176 & 177 & 178 & 179 & 180 & 181 & 182 & 183 \\
\hline 16 & - & - & - & - & - & - & - & $<$ & - & $<$ & - & - & $"="$ & $>$ & $<$ \\
\hline 17 & $>$ & - & - & - & - & - & - & - & - & - & - & - & - & - & - \\
\hline 18 & $>$ & - & - & - & - & - & - & $<$ & - & $<$ & - & $<$ & - & - & - \\
\hline 19 & - & - & - & - & - & - & - & $<$ & - & $<$ & - & $<$ & $<$ & - & $<$ \\
\hline 20 & - & - & - & $>$ & - & $>$ & - & - & - & - & - & - & - & $>$ & - \\
\hline 21 & - & - & - & - & - & - & - & - & - & - & - & - & - & - & - \\
\hline 22 & $>$ & $>$ & - & - & - & - & - & - & - & $<$ & - & $<$ & - & - & - \\
\hline 23 & - & - & $>$ & - & - & $>$ & $>$ & - & - & - & - & - & - & - & - \\
\hline 24 & - & - & - & - & $<$ & $>$ & - & - & - & $<$ & - & - & - & - & - \\
\hline 25 & - & - & - & - & - & - & - & $<$ & - & $<$ & - & - & - & - & - \\
\hline 26 & - & - & - & - & - & - & - & $<$ & - & $<$ & - & - & $<$ & - & $<$ \\
\hline 27 & - & - & - & - & - & $>$ & - & - & - & - & - & - & - & - & - \\
\hline 28 & - & - & - & - & - & - & - & $<$ & - & $<$ & - & - & - & - & - \\
\hline 29 & - & - & - & - & - & - & - & - & - & - & - & - & - & - & - \\
\hline 30 & - & - & - & - & - & - & - & - & - & $<$ & - & - & - & - & - \\
\hline 31 & - & - & $>$ & $>$ & $>$ & $>$ & $>$ & - & - & $<$ & - & - & - & - & - \\
\hline 32 & - & - & $>$ & $>$ & $>$ & $>$ & $>$ & - & - & $<$ & - & - & $>$ & $>$ & $>$ \\
\hline 33 & $>$ & $>$ & - & - & - & - & - & - & - & $<$ & - & - & $>$ & $>$ & $>$ \\
\hline 34 & $>$ & $>$ & $>$ & $>$ & $>$ & $>$ & $>$ & $>$ & $>$ & $>$ & $>$ & $>$ & $>$ & $>$ & $>$ \\
\hline 35 & - & - & - & - & - & - & - & - & - & - & - & - & - & - & - \\
\hline 36 & - & - & - & - & - & - & - & - & - & $<$ & - & - & - & - & - \\
\hline 37 & - & - & $>$ & $>$ & $>$ & $>$ & $>$ & - & - & $<$ & - & - & - & - & - \\
\hline 38 & - & - & - & - & - & - & - & - & - & - & - & - & - & - & - \\
\hline 39 & - & - & - & - & - & - & - & - & - & $<$ & - & - & - & - & - \\
\hline 40 & - & - & $>$ & $>$ & $"="$ & $>$ & $>$ & - & - & $<$ & - & - & - & - & - \\
\hline 41 & - & - & - & - & - & - & - & - & - & $<$ & - & - & $>$ & $>$ & $"="$ \\
\hline 42 & - & - & $>$ & $>$ & $>$ & $>$ & $>$ & - & - & - & - & - & $>$ & $>$ & $>$ \\
\hline 43 & - & - & - & - & - & - & - & - & - & $<$ & - & - & $>$ & $>$ & $>$ \\
\hline 44 & - & - & - & - & - & - & - & - & - & $<$ & - & - & $>$ & $>$ & $>$ \\
\hline 45 & - & - & - & - & - & - & - & - & - & - & - & - & - & - & - \\
\hline 46 & $>$ & - & - & - & - & - & - & $<$ & $<$ & $<$ & $<$ & $<$ & - & - & - \\
\hline 47 & - & - & - & - & - & $>$ & - & $<$ & $<$ & $<$ & $<$ & $<$ & - & - & - \\
\hline 48 & - & - & - & - & - & $>$ & - & $<$ & $<$ & $<$ & $<$ & $<$ & - & - & - \\
\hline 49 & $>$ & - & - & - & - & $>$ & - & - & - & - & $>$ & - & - & - & - \\
\hline 50 & $>$ & - & - & - & - & - & - & - & - & - & - & - & - & - & - \\
\hline 51 & $<$ & $<$ & - & - & - & - & - & $<$ & $<$ & $<$ & $<$ & $<$ & - & - & - \\
\hline 52 & - & - & - & - & - & $>$ & - & $<$ & $<$ & $<$ & $<$ & $<$ & - & - & - \\
\hline \multicolumn{16}{|c|}{ (Continued on next page) } \\
\hline
\end{tabular}


Table 13 (Continued from previous page)

\begin{tabular}{|c|c|c|c|c|c|c|c|c|c|c|c|c|c|c|c|}
\hline & 169 & 170 & 171 & 172 & 173 & 174 & 175 & 176 & 177 & 178 & 179 & 180 & 181 & 182 & 183 \\
\hline 53 & - & - & - & - & - & - & - & - & - & - & - & - & - & - & - \\
\hline 54 & - & - & - & - & - & - & - & $<$ & $<$ & $<$ & $<$ & $<$ & - & - & - \\
\hline 55 & - & - & $<$ & $<$ & $<$ & $"="$ & $<$ & $<$ & $<$ & $<$ & $<$ & $<$ & - & - & - \\
\hline 56 & - & - & - & - & - & - & - & $<$ & $<$ & $<$ & $<$ & $<$ & $<$ & $<$ & $<$ \\
\hline 57 & - & - & - & - & - & $>$ & - & - & - & - & - & - & - & - & - \\
\hline 58 & - & - & - & - & - & - & - & $<$ & $<$ & $<$ & $<$ & $<$ & - & - & - \\
\hline 59 & - & - & - & - & - & - & - & $<$ & $<$ & $<$ & $<$ & $<$ & - & - & - \\
\hline 60 & - & - & - & - & - & - & - & - & - & - & - & - & - & - & - \\
\hline 61 & - & - & - & - & - & - & - & $<$ & - & $<$ & - & - & - & - & - \\
\hline 62 & - & - & - & - & - & $>$ & $>$ & - & - & $<$ & - & - & - & - & - \\
\hline 63 & - & - & - & - & - & $>$ & $>$ & - & - & $<$ & - & - & - & - & - \\
\hline 64 & - & - & - & - & - & - & - & - & - & - & - & - & - & - & - \\
\hline 65 & - & - & - & - & - & $>$ & $>$ & - & - & $<$ & - & - & - & - & - \\
\hline 66 & - & - & - & - & - & - & - & - & - & - & - & - & - & - & - \\
\hline 67 & - & - & - & - & - & - & - & $<$ & - & $<$ & - & - & - & - & - \\
\hline 68 & - & - & - & - & - & $>$ & $>$ & - & - & $<$ & - & - & - & - & - \\
\hline 69 & - & - & - & - & - & $>$ & - & - & $<$ & $<$ & - & - & - & - & - \\
\hline 70 & - & - & - & - & - & - & - & - & - & $<$ & - & - & - & - & - \\
\hline 71 & $>$ & - & - & - & - & - & - & - & - & - & - & - & - & - & - \\
\hline 72 & $>$ & - & - & - & - & - & - & $<$ & $<$ & $<$ & - & - & - & - & - \\
\hline 73 & - & - & - & - & - & - & - & $<$ & $<$ & $<$ & - & - & - & - & - \\
\hline 74 & - & - & - & - & - & $>$ & $>$ & - & - & - & - & - & - & - & - \\
\hline 75 & - & - & - & - & - & - & - & - & - & - & - & - & - & - & - \\
\hline 76 & $>$ & - & - & - & - & - & - & - & $<$ & $<$ & - & - & - & $>$ & - \\
\hline 77 & - & - & $>$ & $>$ & - & $>$ & - & - & - & - & - & - & - & - & - \\
\hline 78 & - & - & - & - & - & - & - & $<$ & - & $<$ & - & - & - & - & - \\
\hline 79 & - & - & - & - & - & $>$ & - & - & - & - & - & - & - & - & - \\
\hline 80 & - & - & - & - & - & - & - & $<$ & - & $<$ & - & - & - & - & - \\
\hline 81 & - & - & - & - & - & - & - & - & - & - & - & - & - & - & - \\
\hline 82 & - & - & - & - & - & - & - & - & - & $<$ & - & - & - & - & - \\
\hline 83 & - & - & $>$ & $>$ & $>$ & $>$ & $>$ & - & - & $<$ & - & - & - & - & - \\
\hline 84 & - & - & $>$ & $>$ & $>$ & $>$ & $>$ & - & - & $<$ & - & - & - & - & - \\
\hline 85 & - & - & - & - & - & - & - & - & - & - & - & - & - & - & - \\
\hline 86 & - & - & - & - & - & - & - & - & - & $<$ & - & - & $>$ & $>$ & $>$ \\
\hline 87 & - & - & - & - & - & - & - & - & - & $<$ & - & - & - & - & - \\
\hline 88 & - & - & $>$ & $>$ & $>$ & $>$ & $>$ & - & - & - & - & - & - & - & - \\
\hline 89 & - & - & - & - & - & - & - & - & - & $<$ & - & - & - & - & - \\
\hline \multicolumn{16}{|c|}{ (Continued on next page) } \\
\hline
\end{tabular}


Table 13 (Continued from previous page)

\begin{tabular}{|c|c|c|c|c|c|c|c|c|c|c|c|c|c|c|c|}
\hline & 169 & 170 & 171 & 172 & 173 & 174 & 175 & 176 & 177 & 178 & 179 & 180 & 181 & 182 & 183 \\
\hline 90 & - & - & - & - & - & - & - & - & - & - & - & - & - & - & - \\
\hline 91 & - & - & - & - & - & - & - & $<$ & $<$ & $<$ & $<$ & $<$ & - & - & - \\
\hline 92 & - & - & - & - & - & $>$ & - & $<$ & $<$ & $<$ & $<$ & $<$ & - & - & - \\
\hline 93 & - & - & - & - & - & $>$ & - & $<$ & $<$ & $<$ & $<$ & $<$ & - & - & - \\
\hline 94 & - & - & - & - & - & - & - & - & - & - & - & - & - & - & - \\
\hline 95 & - & - & - & - & - & - & - & $<$ & $<$ & $<$ & $<$ & $<$ & - & - & - \\
\hline 96 & - & - & - & - & - & - & - & $<$ & $<$ & $<$ & $<$ & $<$ & - & - & - \\
\hline 97 & - & - & - & - & - & $>$ & - & - & - & - & - & - & - & - & - \\
\hline 98 & - & - & - & - & - & - & - & $<$ & $<$ & $<$ & $<$ & $<$ & - & - & - \\
\hline 99 & - & - & - & - & - & - & - & - & - & - & - & - & - & - & - \\
\hline 100 & - & - & - & - & - & - & - & - & - & - & - & - & - & - & - \\
\hline 101 & - & - & - & - & - & - & - & - & - & - & - & - & - & - & - \\
\hline 102 & - & - & - & - & - & - & - & - & - & - & - & - & - & - & - \\
\hline 103 & - & - & - & - & - & - & - & - & - & - & - & - & - & - & - \\
\hline 104 & - & - & - & - & - & - & - & - & - & - & - & - & - & - & - \\
\hline 105 & - & - & - & - & - & - & - & - & - & - & - & - & - & - & - \\
\hline 106 & - & - & - & - & - & - & - & - & - & - & - & - & - & - & - \\
\hline 107 & - & - & - & - & - & - & - & - & - & - & - & - & - & - & - \\
\hline 108 & - & - & - & - & - & - & - & - & - & - & - & - & - & - & - \\
\hline 109 & - & - & - & - & - & - & - & - & - & - & - & - & - & - & - \\
\hline 110 & $>$ & - & - & - & - & - & - & - & - & - & - & - & - & - & - \\
\hline 111 & - & - & - & - & - & - & - & - & - & - & - & - & - & - & - \\
\hline 112 & $>$ & - & - & - & - & - & - & - & - & - & - & - & - & - & - \\
\hline 113 & - & - & - & - & - & - & - & - & - & - & - & - & - & - & - \\
\hline 114 & - & - & - & - & - & - & - & - & - & - & - & - & - & - & - \\
\hline 115 & $>$ & $>$ & - & - & - & - & - & - & - & - & - & - & $>$ & $>$ & $>$ \\
\hline 116 & - & - & - & - & - & - & - & - & - & - & - & - & - & - & - \\
\hline 117 & - & - & - & - & - & - & - & - & - & - & - & - & - & - & - \\
\hline 118 & - & - & - & - & - & - & - & - & - & - & - & - & - & - & - \\
\hline 119 & - & - & - & - & - & - & - & - & - & - & - & - & - & - & - \\
\hline 120 & $>$ & - & - & - & - & - & - & - & - & - & - & - & - & - & - \\
\hline 121 & - & - & - & - & - & - & - & - & - & - & - & - & - & - & - \\
\hline 122 & $>$ & - & - & - & - & - & - & - & - & - & - & - & - & - & - \\
\hline 123 & - & - & - & - & - & - & - & - & - & - & - & - & - & - & - \\
\hline 124 & - & - & - & - & - & - & - & - & - & - & - & - & - & - & - \\
\hline 125 & $>$ & - & - & - & - & - & - & - & - & - & - & - & - & - & - \\
\hline 126 & - & - & - & - & - & - & - & - & - & - & - & - & - & - & - \\
\hline \multicolumn{16}{|c|}{ (Continued on next page) } \\
\hline
\end{tabular}


Table 13 (Continued from previous page)

\begin{tabular}{|c|c|c|c|c|c|c|c|c|c|c|c|c|c|c|c|}
\hline & 169 & 170 & 171 & 172 & 173 & 174 & 175 & 176 & 177 & 178 & 179 & 180 & 181 & 182 & 183 \\
\hline 127 & $>$ & - & - & - & - & - & - & - & - & - & - & - & - & - & - \\
\hline 128 & - & - & - & - & - & - & - & - & - & - & - & - & - & - & - \\
\hline 129 & - & - & - & - & - & - & - & - & - & - & - & - & - & - & - \\
\hline 130 & $>$ & $>$ & - & - & - & - & - & - & - & - & - & - & $>$ & $>$ & $>$ \\
\hline 131 & - & - & - & - & - & - & - & - & - & - & - & - & $>$ & $>$ & $>$ \\
\hline 132 & - & - & - & - & - & - & - & - & - & - & - & - & - & - & - \\
\hline 133 & - & - & - & - & - & - & - & - & - & - & - & - & - & - & - \\
\hline 134 & - & - & - & - & - & - & - & - & - & - & - & - & - & - & - \\
\hline 135 & $>$ & - & - & - & - & - & - & - & - & - & - & - & - & - & - \\
\hline 136 & - & - & - & - & - & - & - & - & - & - & - & - & - & - & - \\
\hline 137 & $>$ & - & - & - & - & - & - & - & - & - & - & - & - & - & - \\
\hline 138 & - & - & - & - & - & - & - & - & - & - & - & - & - & - & - \\
\hline 139 & - & - & - & - & - & - & - & $<$ & - & $<$ & - & - & - & - & - \\
\hline 140 & - & - & - & - & - & - & - & $<$ & - & $<$ & - & - & - & - & - \\
\hline 141 & - & - & - & - & - & - & - & $<$ & - & $<$ & - & - & - & - & - \\
\hline 142 & - & - & - & - & - & - & - & - & - & $<$ & - & - & - & - & - \\
\hline 143 & - & - & - & - & - & - & - & - & $<$ & $<$ & - & - & - & - & - \\
\hline 144 & - & - & - & - & - & - & - & - & - & $<$ & - & $<$ & - & - & - \\
\hline 145 & - & - & - & - & - & - & - & $<$ & $<$ & $<$ & $<$ & $<$ & - & - & - \\
\hline 146 & - & - & - & - & - & - & - & - & - & $<$ & - & - & - & - & - \\
\hline 147 & - & - & - & - & - & - & - & - & $<$ & $<$ & - & - & - & - & - \\
\hline 148 & - & - & - & - & - & - & - & - & - & $<$ & - & $<$ & - & - & - \\
\hline 149 & - & - & - & - & - & - & - & $<$ & $<$ & $<$ & $<$ & $<$ & - & - & - \\
\hline 166 & $<$ & $<$ & $<$ & $<$ & $<$ & $<$ & $<$ & $<$ & $<$ & $<$ & $<$ & $<$ & $<$ & - & - \\
\hline 167 & - & - & - & - & - & - & - & - & $<$ & $<$ & - & - & - & - & - \\
\hline 168 & - & - & - & - & - & - & - & - & - & $<$ & - & - & - & - & - \\
\hline 169 & $=$ & $<$ & - & - & - & - & - & $<$ & $<$ & $<$ & $<$ & $<$ & - & - & - \\
\hline 170 & $>$ & $=$ & - & - & - & - & - & - & - & $<$ & - & $<$ & - & - & - \\
\hline 171 & - & - & $=$ & - & $<$ & $>$ & - & - & - & $<$ & - & - & - & - & - \\
\hline 172 & - & - & - & $=$ & $<$ & $>$ & - & $<$ & $<$ & $<$ & - & - & - & - & - \\
\hline 173 & - & - & $>$ & $>$ & $=$ & $>$ & $>$ & - & - & $<$ & - & - & - & - & - \\
\hline 174 & - & - & $<$ & $<$ & $<$ & $=$ & $<$ & $<$ & $<$ & $<$ & $<$ & $<$ & - & - & - \\
\hline 175 & - & - & - & - & $<$ & $>$ & $=$ & - & - & $<$ & - & $<$ & - & - & - \\
\hline 176 & $>$ & - & - & $>$ & - & $>$ & - & $=$ & - & $<$ & $>$ & - & $>$ & $>$ & - \\
\hline 177 & $>$ & - & - & $>$ & - & $>$ & - & - & $=$ & $<$ & $>$ & - & - & $>$ & - \\
\hline 178 & $>$ & $>$ & $>$ & $>$ & $>$ & $>$ & $>$ & $>$ & $>$ & $=$ & $>$ & $>$ & $>$ & $>$ & $>$ \\
\hline 179 & $>$ & - & - & - & - & $>$ & - & $<$ & $<$ & $<$ & $=$ & $<$ & - & - & - \\
\hline \multicolumn{16}{|c|}{ (Continued on next page) } \\
\hline
\end{tabular}


Table 13 (Continued from previous page)

\begin{tabular}{|l|ccccccccccccccc|}
\hline & 169 & 170 & 171 & 172 & 173 & 174 & 175 & 176 & 177 & 178 & 179 & 180 & 181 & 182 & 183 \\
\hline 180 & $>$ & $>$ & - & - & - & $>$ & $>$ & - & - & $<$ & $>$ & $=$ & - & - & - \\
\hline 181 & - & - & - & - & - & - & - & $<$ & - & $<$ & - & - & $=$ & $>$ & $<$ \\
\hline 182 & - & - & - & - & - & - & - & $<$ & $<$ & $<$ & - & - & $<$ & $=$ & $<$ \\
\hline 183 & - & - & - & - & - & - & - & - & - & $<$ & - & - & $>$ & $>$ & $=$ \\
\hline 184 & - & - & - & - & - & - & - & - & - & $<$ & - & $<$ & - & - & $<$ \\
\hline 185 & - & - & - & - & - & - & - & $<$ & $<$ & $<$ & $<$ & $<$ & $<$ & $<$ & $<$ \\
\hline 186 & $>$ & - & - & - & - & $>$ & $>$ & - & - & $<$ & $>$ & - & - & - & - \\
\hline 187 & $>$ & $>$ & - & - & - & $>$ & $>$ & - & - & $<$ & $>$ & $="$ & - & - & - \\
\hline 188 & $>$ & $>$ & $>$ & $>$ & $>$ & $>$ & $>$ & $>$ & $>$ & $\prime=$ & $>$ & $>$ & $>$ & $>$ & $>$ \\
\hline 189 & $>$ & - & - & - & - & $>$ & - & $<$ & $<$ & $<$ & $="$ & $<$ & - & - & - \\
\hline 190 & $>$ & $>$ & - & - & - & $>$ & $>$ & - & - & $<$ & $>$ & $="$ & - & - & - \\
\hline 191 & - & - & - & $>$ & - & $>$ & - & - & - & - & - & - & - & $>$ & - \\
\hline 192 & - & - & - & - & - & - & - & $<$ & - & $<$ & - & - & - & - & - \\
\hline 193 & - & - & - & - & - & - & - & $<$ & $<$ & $<$ & $<$ & $<$ & - & - & - \\
\hline 194 & - & - & - & - & - & - & - & - & - & $<$ & - & - & - & - & - \\
\hline 195 & - & - & - & - & - & - & - & - & $<$ & $<$ & - & - & - & - & - \\
\hline 196 & - & - & - & - & - & - & - & - & - & $<$ & - & - & - & - & - \\
\hline 197 & - & - & - & - & - & - & - & $<$ & $<$ & $<$ & $<$ & $<$ & - & - & - \\
\hline 198 & - & - & - & - & - & - & - & - & - & $<$ & - & $<$ & - & - & - \\
\hline
\end{tabular}

In the research, we have eliminated the implications $\rightarrow_{150}, \ldots, \rightarrow_{165}$, because they contain parameters and cannot be compared with the other implications. An Open problem is to search for the relations between these parametric implications and what are the relations between them (for different values of their parameters) and the rest of the implications.

In the cell with coordinates $(i, j)$ there is one of the symbols $<,>,=, "=$ " or - . The first symbol corresponds to the case $x \rightarrow_{i} y<x \rightarrow_{j} y$, second symbol - to the case $x \rightarrow_{i} y>x \rightarrow_{j}$ $y$, third - to $x \rightarrow_{i} y=x \rightarrow_{i} y$ (that is the normal situation), the fourth symbol - to the case $x \rightarrow_{i} y=x \rightarrow_{j} y$ for $i \neq j$, while the fifth symbol - to the case when there is none of the three possible relations. Here, $1 \leq i, j \leq 198$.

When the program determines symbols $<$, $>$ or - , then between both implications there is one of the two relation $<,>$, or there is none of the them. But when the symbol is " = ", a formal check is necessary that both implications coincide. So, we check validity for the cases, when the symbol " = "stays in the cell with coordinates $(i, j)$ and $i \neq j$, for example, for the case $i=16, j=181$ (or $i=181, j=16$; this symmetry holds in all cases with symbol " =" and by this reason below we will not mention this fact).

Let

$$
X \equiv 1-\operatorname{sg}(a) \cdot(1-c)-\max (\overline{s g}(a), c)
$$


If $a=0$, then

$$
x=1-0 .(1-c)-\max (1, c)=1-1=0 .
$$

If $a>0$, then

$$
X=1-1 .(1-c)-\max (0, c)=1-1+c-c=0 .
$$

Therefore, the first components of the result of implications $\rightarrow_{16}$ and $\rightarrow_{181}$ coincide.

Let

$$
Y \equiv \min (\operatorname{sg}(a), d)-d \cdot \operatorname{sg}(a)
$$

If $a=0$, then

$$
Y=\min (0, d)-d .0=0
$$

If $a>0$, then

$$
Y=\min (1, d)-d .1=d-d=0 .
$$

Therefore, the second components of the result of implications $\rightarrow_{16}$ and $\rightarrow_{181}$ coincide, too, i.e., both implications coincide.

The checks of coincidence of $\rightarrow_{40}$ and $\rightarrow_{173}$; of $\rightarrow_{41}$ and $\rightarrow_{183}$; of $\rightarrow_{43}$ and $\rightarrow_{44}$; of $\rightarrow_{55}$ and $\rightarrow_{174}$; of $\rightarrow_{56}$ and $\rightarrow_{185}$; of $\rightarrow_{178}$ and $\rightarrow_{188}$; of $\rightarrow_{179}$ and $\rightarrow_{189}$; and of $\rightarrow_{180}, \rightarrow_{187}$ and $\rightarrow_{190}$ are done in the same manner.

The reason for these coincidences is that they are generated by different time-moments and in some case, by different authors. In practice, the idea for searching of coincidences was generated now and this is the first systematic check. In [59] one coincidence was found by P. Vassilev, S. Ribagin and J. Kacprzyk. Here, we show that the number of these coincidences is larger.

\section{Conclusion: ideas for the future}

Our idea for the present check is the first step of a more general research. Using the results from Table 1, we will construct, by analogy with [13], oriented graphs with vertices corresponding to the different implications and oriented arcs with directions determined by the symbols of the Table. After this, we will search these elements of the graphs that satisfy the axioms for implications, checked in [16]. Finally, we will keep only the implication with best properties and for them, we will give new numeration, different from the one in $[13,16]$.

\section{Acknowledgement}

This research was funded by the Bulgarian National Science Fund, grant number KP-06-N22/1/ 2018 "Theoretical research and applications of InterCriteria Analysis". 


\section{References}

[1] Angelova, N. (2019). IFSTOOL - Software for intuitionistic fuzzy sets - Necessity, Possibility and Circle operators. Advances in Intelligent Systems and Computing, issue:1081, Springer, 76-81.

[2] Angelova, N., \& Atanassov, K. (2015). Intuitionistic Fuzzy Implications and the Axioms of Intuitionistic Logic. In:Proc. of the 9th Conference of the European Society for Fuzzy Logic and Technology (EUSFLAT), 30.06-03.07.2015, Gijon, Spain, 1578-1584.

[3] Angelova, N., \& Atanassov, K. (2016). Intuitionistic Fuzzy Implications and Klir-Yuan's Axioms.Novel Developments in Uncertainty Representation and Processing. Advances in Intuitionistic Fuzzy Sets and Generalized Nets, Advances in Intelligent Systems and Computing, 401. Atanassov, K.T., Castillo, O., Kacprzyk, J., Krawczak, M., Melin, P., Sotirov, S., Sotirova, E., Szmidt, E., De Tré, G., Zadrożny, S. (Eds.), 97-110.

[4] Angelova, N., Marinov, E., \& Atanassov, K. (2015). Intuitionistic fuzzy implications and Kolmogorov's and Łukasiewisz-Tarski's axioms of logic. Notes on Intuitionistic Fuzzy Sets, $21(2), 35-42$.

[5] Atanassov, K. (1988). Two variants of intuitonistc fuzzy propositional calculus. Preprint IM-MFAIS-5-88, Sofia.

[6] Atanassov, K. (2005). On some intuitionistic fuzzy negations. Proc. of the First Int. Workshop on IFSs, Banska Bystrica, 22 Sept. 2005. Notes on Intuitionistic Fuzzy Sets, 11 (6), 13-20.

[7] Atanassov, K. (2006). On some intuitionistic fuzzy implications. Comptes Rendus de l'Academie bulgare des Sciences, 59 (1), 19-24.

[8] Atanassov, K. (2006). A new intuitionistic fuzzy implication from a modal type. Advanced Studies on Contemporary Mathematics, 12 (1), 117-122.

[9] Atanassov, K. (2006). On eight new intuitionistic fuzzy implications. Proc. of 3rd Int. IEEE Conf. “Intelligent Systems” ISO6, London, 4-6 Sept. 2006, 741-746.

[10] Atanassov, K. (2008). On intuitionistic fuzzy implication $\rightarrow^{\varepsilon}$ and intuitionistic fuzzy negation $\neg^{\varepsilon}$. Issues in Intuitionistic Fuzzy Sets and Generalized Nets, 6, 6-19.

[11] Atanassov, K. (2008). Intuitionistic fuzzy implication $\rightarrow^{\varepsilon, \eta}$ and intuitionistic fuzzy negation $\neg^{\varepsilon, \eta}$. Developments in Fuzzy Sets, Intuitionistic Fuzzy Sets, Generalized Nets and Related Topics, 1, 1-10.

[12] Atanassov, K. (2011). Second Zadeh's intuitionistic fuzzy implication. Notes on Intuitionistic Fuzzy Sets. 17 (3), 11-14.

[13] Atanassov, K. (2012). On Intuitionistic Fuzzy Sets Theory, Springer, Berlin. 
[14] Atanassov, K. (2015). On a New Intuitionistic Fuzzy Implication. In: Proc of the 9th Conference of the European Society for Fuzzy Logic and Technology (EUSFLAT), 30.06-03.07.2015, Gijon, Spain, 1592-1597.

[15] Atanassov, K. (2016). On intuitionistic fuzzy implications, Issues in Intuitionistic Fuzzy Sets and Generalized Nets, 12, 1-19.

[16] Atanassov, K. (2017). Intuitionistic Fuzzy Logics, Springer, Cham.

[17] Atanassov, K (2021). Third Zadeh's Intuitionistic Fuzzy Implication. Mathematics, 9, 619. https://doi.org/10.3390/math9060619

[18] Atanassov, K., \& Angelova, N. (2021). Modifications of the Third Zadeh's intuitionistic fuzzy implication. Notes on Intuitionistic Fuzzy Sets, 27 (1), 9-23.

[19] Atanassov, K.,\& Angelova, N. (2016). Properties of intuitionistic fuzzy implications and negations. Notes on Intuitionistic Fuzzy Sets, 22 (3) , 25-33.

[20] Atanassov, K., Angelova, N. \& Atanassova, V. (2021). On an Intuitionistic Fuzzy Form of the Goguen's Implication. Mathematics, 9, 676. https://doi.org/10.3390/math9060676

[21] Atanassov, K., \& Dimitrov, D. (2010). Intuitionistic fuzzy implications and axioms for implications. Notes in Intuitionistic Fuzzy Sets, 16, (1), 10-20.

[22] Atanassov, K., \& Kolev, B. (2006). On an intuitionistic fuzzy implication from a probabilistic type. Advanced Studies on Contemporary Mathematics, 12 (1), 111-116.

[23] Atanassov, K., S. Ribagin, L. Doukovska, \& V. Atanassova (2017). Intuitionistic fuzzy implication $\rightarrow_{190}$. Notes on Intuitionistic Fuzzy Sets, 23 (4), 79-83.

[24] Atanassov, K., \& Szmidt, E. (2014). Remark on intuitionistic fuzzy implication $\rightarrow^{\varepsilon, \eta}$. Issues in Intuitionistic Fuzzy Sets and Generalized Nets, 11, 9-14.

[25] Atanassov, K., Szmidt, E., \& Angelova, N.(2017). Properties of the intuitionistic fuzzy implication $\rightarrow_{187}$. Notes on Intuitionistic Fuzzy Sets, 23 (3), 3-8.

[26] Atanassov, K., Szmidt, E., \& Kacprzyk, J. (2013). On intuitionistic fuzzy pairs. Notes on Intuitionistic Fuzzy Sets, 19 (3), 1-13.

[27] Atanassov, K., Szmidt, E., \& Kacprzyk, J. (2015). On Fodor's type of intuitionistic fuzzy implication and negation. Notes on Intuitionistic Fuzzy Sets, 21 (2), 25-34.

[28] Atanassov, K., Szmidt, E., \& Kacprzyk, J. (2016). New Fodor's Type Of Intuitionistic Fuzzy Implication and Negation. Notes on Intuitionistic Fuzzy Sets, 22 (3), 1-8.

[29] Atanassov, K., Szmidt, E., \& Kacprzyk, J. (2017). On intuitionistic fuzzy implication $\rightarrow_{187}$. Notes on Intuitionistic Fuzzy Sets, 23 (2), 37-43. 
[30] Atanassov, K., Szmidt, E., \& Kacprzyk, J. (2017). On intuitionistic fuzzy implication $\rightarrow_{188}$. Notes on Intuitionistic Fuzzy Sets, 23 (1), 6-13.

[31] Atanassov, K., Szmidt, E., Kacprzyk, J., \& Angelova, N. (2019). Intuitionistic fuzzy implications revisited. Part 1. Notes on Intuitionistic Fuzzy Sets, 25(3), 71-78.

[32] Atanassov, K., \& Trifonov, T. (2005). On a new intuitionistic fuzzy implication of Godel's type. Proceedings of the Jangjeon Mathematical Society, 8 (2), 147-152.

[33] Atanassov, K., \& Trifonov, T. (2006). Two new intuitionistic fuzzy implications. Advanced Studies on Contemporary Mathematics, 13 (1), 69-74.

[34] Atanassova, L. (2008). On an intuitionistic fuzzy implication from Kleene-Dienes type. Proceedings of the Jangjeon Mathematical Society, 11 (1), 69-74.

[35] Atanassova, L. (2008). Modifications of an intuitionistic fuzzy implication from Kleene-Dienes type. Advanced Studies in Contemporary Mathematics, 16 (2), 155-160.

[36] Atanassova, L. (2008). New modifications of an intuitionistic fuzzy implication from Kleene-Dienes type. Part 2. Annual of Section "Informatics", 1, 59-64.

[37] Atanassova, L. (2009). New modifications of an intuitionistic fuzzy implication from Kleene-Dienes type. Part 3. Advanced Studies in Contemporary Mathematics, 18 (1), 33-40.

[38] Atanassova, L. (2009). A new intuitionistic fuzzy implication. Cybernetics and Information Technologies, 9 (2), 21-25.

[39] Atanassova, L. (2009). On some properties of intuitionistic fuzzy negation $\neg$ @. Notes on Intuitionistic Fuzzy Sets, 15 (1), 32-35.

[40] Atanassova, L. (2012). On two modifications of the intuitionistic fuzzy implication $\rightarrow @$. Notes on Intuitionistic Fuzzy Sets, 18 (2), 26-30.

[41] Atanassova, L. (2013). On the modal form of the intuitionistic fuzzy implications $\rightarrow_{@}^{\prime}$ and $\rightarrow$ @. Issues in Intuitionistic Fuzzy Sets and Generalized Nets, 10, 5-11.

[42] Atanassova, L. (2013). On the intuitionistic fuzzy form of the classical implication $(A \rightarrow$ $B) \vee(B \rightarrow A)$. Notes on Intuitionistic Fuzzy Sets, 19 (4), 15-18.

[43] Atanassova, L. (2014). Remark on the intuitionistic fuzzy forms of two classical logic axioms. Part 1. Annual of Section "Informatics", 7, 24-27.

[44] Atanassova, L. (2014). Remark on the intuitionistic fuzzy forms of two classical logic axioms. Part 2. Notes on Intuitionistic Fuzzy Sets, 20 (4), 10-13.

[45] Atanassova, L. (2015). Remark on Dworniczak's intuitionistic fuzzy implications. Part 1. Notes on Intuitionistic Fuzzy Sets, 21 (3), 18-23. 
[46] Atanassova, L. (2015) Remark on Dworniczak’s intuitionistic fuzzy implications. Part 2. Issues in Intuitionistic Fuzzy Sets and Generalized Nets, 61-67.

[47] Atanassova, L. (2016). Remark on Dworniczak's intuitionistic fuzzy implications. Part 3. Notes on Intuitionistic Fuzzy Sets, 22 (1), 1-6.

[48] Atanassova, L. (2017). Intuitionistic fuzzy implication $\rightarrow_{189}$. Notes on Intuitionistic Fuzzy Sets, 2 (1), 14-20.

[49] Atanassova, L. (2017). Properties of the intuitionistic fuzzy implication $\rightarrow_{189}$. Notes on Intuitionistic Fuzzy Sets, 23 (4), 10-14.

[50] Dworniczak, P. (2010). Some remarks about the L. Atanassova's paper "A new intuitionistic fuzzy implication”. Cybernetics and Information Technologies, 10 (3), 3-9.

[51] Dworniczak, P. (2010). On one class of intuitionistic fuzzy implications. Cybernetics and Information Technologies, 10 (4), 13-21.

[52] Dworniczak, P. (2011). On some two-parametric intuitionistic fuzzy implication. Notes on Intuitionistic Fuzzy Sets, 17 (2), 8-16.

[53] Feys, R. (1965). Modal Logics. Gauthier-Villars, Paris.

[54] Klir, G., \& Yuan, B. (1995). Fuzzy Sets and Fuzzy Logic. Prentice Hall, New Jersey.

[55] Riecan, B., \& Atanassov., K. (2007). On a new intuitionistic fuzzy implication of Gaines-Rescher's type. Notes on Intuitionistic Fuzzy Sets, 13 (4), 1-4.

[56] Szmidt, E., Kacprzyk, J., \& Atanassov, K. (2015). Modal forms of Fodor's type of intuitionistic fuzzy implication. Notes on Intuitionistic Fuzzy Sets, 21 (5), 1-5.

[57] Szmidt, E., Kacprzyk, J., \& Atanassov, K. (2015). Properties of Fodor's intuitionistic fuzzy implication and negation. Notes on Intuitionistic Fuzzy Sets, 21 (4), 6-12.

[58] Vassilev, P., \& Atanassov, K. (2019). Extensions and Modifications of Intuitionistic Fuzzy Sets. "Prof. Marin Drinov" Academic Publishing House, Sofia.

[59] Vassilev, P., Ribagin, S., \& Kacprzyk, J. (2018). A remark on intuitionistic fuzzy implications. Notes on Intuitionistic Fuzzy Sets, 24 (2), 1-7. 